\title{
GEOMETRICAL EVOLUTION OF DEVELOPED INTERFACES
}

\author{
PIERO DE MOTTONI AND MICHELLE SCHATZMAN
}

\begin{abstract}
Consider the reaction-diffusion equation in $\mathbb{R}^{N} \times \mathbb{R}^{+}: u_{t}-h^{2} \Delta u+$ $\varphi(u)=0 ; \varphi$ is the derivative of a bistable potential with wells of equal depth and $h$ is a small parameter. If the initial data has an interface, we give an asymptotic expansion of arbitrarily high order and error estimates valid up to time $O\left(h^{-2}\right)$. At lowest order, the interface evolves normally, with a velocity proportional to the mean curvature.
\end{abstract}

RÉsumE. Soit l'équation de réaction-diffusion dans $\mathbb{R}^{N} \times \mathbb{R}^{+}, u_{t}-h^{2} \Delta u+$ $\varphi(u)=0$, avec $\varphi$ la dérivée d'un potentiel bistable à puits également profonds et $h$ un petit paramètre. Pour une condition initiale possédant une interface, on donne un développement asymptotique d'ordre arbitrairement élevé, ainsi que des estimations d'erreur valides jusqu'à un temps en $O\left(h^{-2}\right)$. A l'ordre le plus bas, l'interface évolue normalement, à une vitesse proportionnelle à la courbure moyenne.

\section{INTRODUCTION}

1.1. Orientation. Let $\Phi(u)$ be an even smooth function, having the shape given in Figure 1.1, and let $\phi=\Phi^{\prime}$. The exact condition satisfied by $\Phi$ and $\phi$ are specified as follows:

$$
\left\{\begin{array}{l}
\phi(u)<0, \forall u \in(0,1), \quad \phi(u)>0, \forall u \in(1,+\infty), \\
\phi^{\prime}(1)>0, \\
r \phi^{\prime \prime}(r)>0, \forall r \neq 0 .
\end{array}\right.
$$

Let $h>0$ be a small parameter; consider the semilinear parabolic equation in $\mathbb{R}^{N} \times \mathbb{R}^{+}, N>1$

$$
\frac{\partial u}{\partial t}-h^{2} \Delta u+\phi(u)=0
$$

Received by the editors May 8, 1991. The present paper was received by the Journal of the AMS in November 1989; a referee's report was sent on August 15, 1990, and received by the first author on September 22, 1990. Requested modifications were sent at the beginning of December 1990, and the paper was rejected in January 1991. The revised version of the paper was sent directly by the editor of $J A M S$ to the editor of the Transactions of the AMS; it was received on May 8, 1991 and accepted on that date.

1980 Mathematics Subject Classification (1985 Revision). Primary 35B25, 35K57; Secondary $53 \mathrm{~A} 10$.

The work of P. de Mottoni has been partially supported by Université Lyon 1-Claude-Bernard; the work of Michelle Schatzman has been partially supported by the Italian Ministry of Universities and Research, National Program on Differential Equations. 


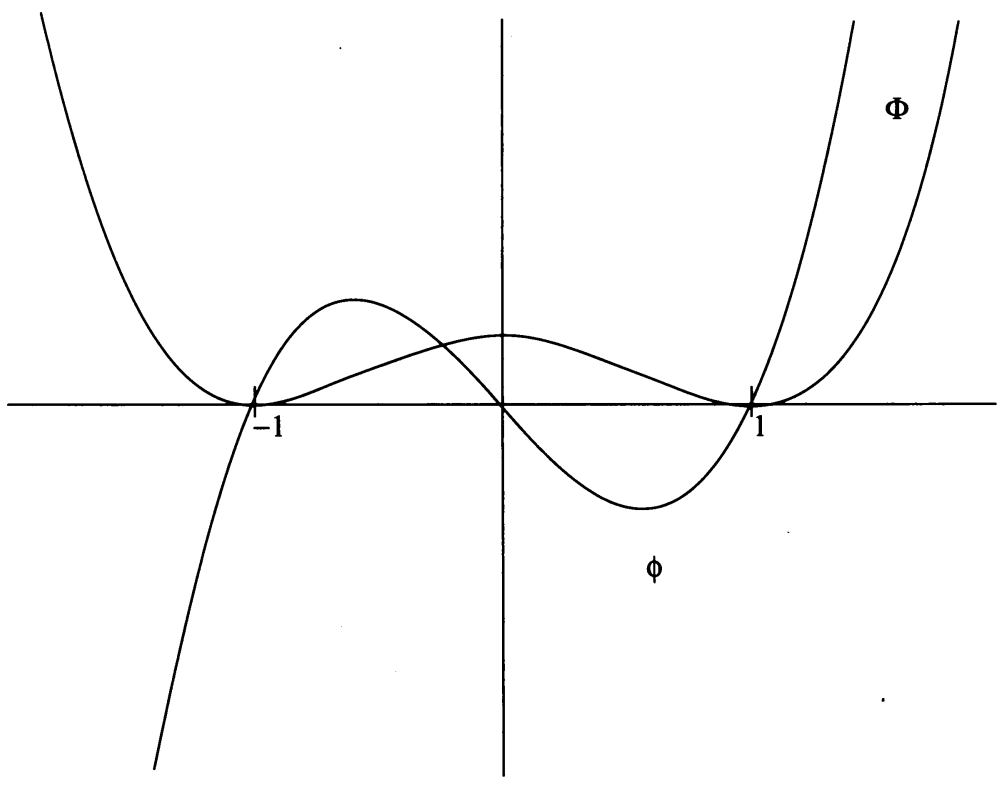

Figure 1.1. The functions $\Phi$ and $\phi$

with initial condition

$$
u(x, 0)=u_{0}(x), \quad x \in \mathbb{R}^{N} .
$$

Our problem arises naturally when studying transition between phases which are equally probable, and one is not interested in the microscopic properties of the interface. Equation (1.2) can be derived from the time-dependent real Ginzburg-Landau model [KaOh], and our $u$ can be understood as an order parameter.

We show that if $u_{0}$ is in $L^{\infty}\left(\mathbb{R}^{N}\right)$ (1.2), (1.3) possesses a unique solution, and we give estimates for large times of all the derivatives of $u(\S 2)$.

For not too large times, the solution of (1.2) with smooth initial data $u_{0}$ behaves as if there were no diffusion. In particular, it tends to \pm 1 , according to the sign of the initial data. We choose initial data which are bounded away from zero for large $|x|$. When the gradient of $u$ is large enough (namely, $|\nabla u|=O(1 / h))$, the diffusion balances the kinetic effects. The solution of (1.2), (1.3) stays smooth because of the diffusion, but it develops transition zones between the regions where $u \sim 1$ and $u \sim-1$, in which $|\nabla u|$ is very large. A dimensional argument shows that the thickness of these transition zones should be $O(h)$.

We are interested in the evolution of developed interfaces for large but not infinite times, "developed" meaning that the initial condition has an interface. The asymptotic for $t \rightarrow+\infty$ is not interesting: if $\liminf _{|x| \rightarrow \infty} u_{0}(x)>0$, then it can be shown that $\lim _{t \rightarrow+\infty} u(x, t)=+1$, and symmetrically with respect to $u=0$.

The phenomena we consider are on the time scale $h^{-2}$, in contrast with the one-dimensional case [CaPe], where the relevant time scale is $\exp (C / h)$. Nevertheless, the simple aspects of the one-dimensional case are the basic building blocks for our analysis. 


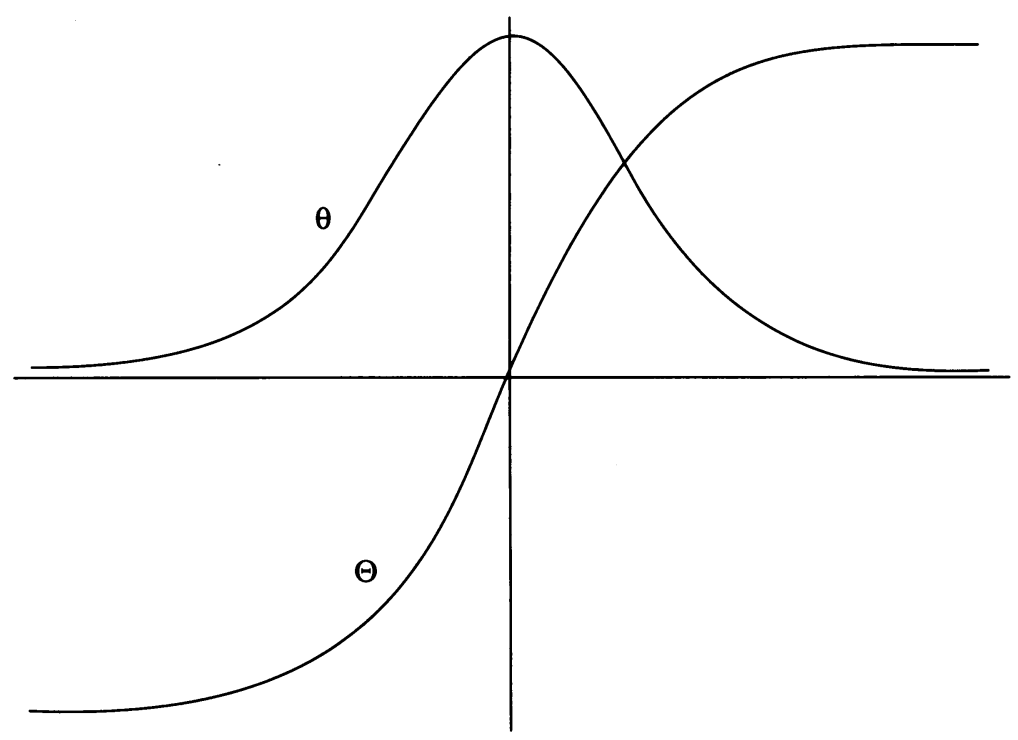

FIGURE 1.2. The standing wave $\Theta$ and its derivative $\theta$

It is well known [Ka] that the problem

$$
\frac{\partial u}{\partial t}-\frac{\partial^{2} u}{\partial x^{2}}+\phi(u)=0, \quad x \in \mathbb{R}
$$

admits travelling wave solutions $v(x-c t)$ which are standing, i.e., $c=0$. This holds because $\Phi$ has minima of equal depth. Let $\Theta$ be the unique increasing solution of

$$
-\boldsymbol{\Theta}^{\prime \prime}+\phi(\boldsymbol{\Theta})=0
$$

such that $\Theta(0)=0$, and let $\theta=\Theta^{\prime}$; see Figure 1.2. Clearly, $\Theta(x)$ converges exponentially to \pm 1 as $x \rightarrow \pm \infty$. More precisely, there exist positive constants $\beta$ and $\beta^{\prime}$ such that:

$$
\left\{\begin{array}{rlrl}
1-\Theta(x) & \sim \beta^{\prime} \exp (-\beta x), & & \text { for } x \rightarrow+\infty, \\
\theta(x) & \sim \beta \beta^{\prime} \exp (-\beta x), & \text { for } x \rightarrow+\infty, \\
\theta^{\prime}(x) & \sim-\beta^{2} \beta^{\prime} \exp (-\beta x), \quad \text { for } x \rightarrow+\infty,
\end{array}\right.
$$

with similar expressions as $x$ tends to $-\infty$.

The linearization of (1.4) around $\Theta$ suggests that we consider the unbounded operator $A$ in $L^{2}(\mathbb{R})$ defined by by

$$
D(A)=H^{2}(\mathbb{R}) ; \quad A u=-u^{\prime \prime}+\phi^{\prime}(\Theta) u .
$$

which is clearly selfadjoint because $\phi^{\prime}(\boldsymbol{\Theta})$ is real and bounded. An essential property of $A$ is summarized in the following statements:

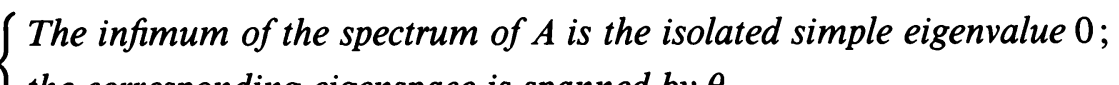

the corresponding eigenspace is spanned by $\theta$.

Indeed, if we differentiate (1.5), we can see that $\theta$ satisfies

$$
-\theta^{\prime \prime}+\phi^{\prime}(\Theta) \theta=0,
$$


i.e., $\theta$ is in the kernel of $A$. As $\phi^{\prime}(1)=\max _{x} \phi^{\prime}(\Theta(x))$, we know from [AcGl] that the intersection of the spectrum of $A$ with $\left(-\infty, \phi^{\prime}(1)\right)$ contains only eigenvalues. Since $\theta$ does not vanish, 0 is a simple eigenvalue.

That zero is the lowest eigenvalue of $A$ is a geometrical fact, which expresses the invariance of (1.4) with respect to space translations.

With the operator $A$ we shall associate the Rayleigh quotient

$$
\bar{R}^{0}[y]=\frac{\int\left(|\nabla y|^{2}+\phi^{\prime}(\boldsymbol{\Theta}) y^{2}\right) d x}{\int y^{2} d x}
$$

defined for $y \in H^{1}\left(\mathbb{R}^{N}\right), y \neq 0$. Here, and throughout the paper, the integralsif not otherwise specified-extend to the whole space. Clearly,

$$
\inf \left\{\bar{R}^{0}[y] / y \in H^{1}\left(\mathbb{R}^{N}\right)\right\}=0 .
$$

It is well known that interfaces appear in the one-dimensional case [FMcL]; in [MoSc2] we show that they develop in the $N$-dimensional case as well.

The first guess for an approximate solution of $(1.2)$ is

$$
u=\boldsymbol{\Theta}\left(\frac{\Lambda^{0}\left(x, h^{2} t\right)}{h}\right),
$$

where $\Lambda^{0}(x, s)$ is the algebraic distance from $x \in \mathbb{R}^{N}$ to a certain hypersurface $\Gamma^{0}\left(h^{2} t\right)$ (namely, $\Lambda^{0}$ is the Euclidean distance on one side of $\Gamma^{0}$ and minus the Euclidean distance on the other side); the slow time scale $s=h^{2} t$ will be justified by the following considerations: If we apply $\partial / \partial t-h^{2} \Delta+\phi$ to (1.11) we obtain

$$
\begin{aligned}
\left(\frac{\partial}{\partial t}-\right. & \left.h^{2} \Delta\right) u+\phi(u) \\
= & h \theta\left(\frac{\Lambda^{0}\left(x, h^{2} t\right)}{h}\right) \frac{\partial}{\partial s} \Lambda^{0}\left(x, h^{2} t\right)-\Theta^{\prime \prime}\left(\frac{\Lambda^{0}\left(x, h^{2} t\right)}{h}\right)\left|\nabla \Lambda^{0}\left(x, h^{2} t\right)\right|^{2} \\
& -h \theta\left(\frac{\Lambda^{0}\left(x, h^{2} t\right)}{h}\right) \Delta \Lambda^{0}\left(x, h^{2} t\right)+\phi(u) .
\end{aligned}
$$
hood

If $\Gamma^{0}(s)$ is smooth, the distance to $\Gamma^{0}(s)$ is smooth in a tubular neighbour-

$$
\mathscr{V}^{0}(s)=\left\{x / \operatorname{dist}\left(x, \Gamma^{0}(s)\right) \leq \bar{\lambda}\right\},
$$

where $\operatorname{dist}(x, A)$ is the Euclidean distance from $x$ to a set $A$, and

$$
\left|\nabla \Lambda^{0}(x, s)\right|=1 \text { in } \mathscr{V}^{0}(s)
$$

Therefore (1.12) becomes by virtue of (1.5)

$$
\begin{aligned}
\left(\frac{\partial}{\partial t}\right. & \left.-h^{2} \Delta\right) u+\phi(u) \\
& =h \theta\left(\frac{\Lambda^{0}\left(x, h^{2} t\right)}{h}\right)\left(\frac{\partial \Lambda^{0}}{\partial s}-\Delta \Lambda^{0}\right)\left(x, h^{2} t\right) .
\end{aligned}
$$

The function $\theta(x)$ takes its maximum at $x=0$. To make the right-hand side of (1.12) as small as possible, it is necessary to have

$$
\left(\frac{\partial \Lambda^{0}}{\partial s}-\Delta \Lambda^{0}\right)(x, s)=0, \quad x \in \Gamma^{0}(s) .
$$


Relations (1.13) and (1.15) together govern the evolution of $\Gamma^{0}(s)$.

As will be shown in subsection 1.2, the lowest order term (1.15) for the evolution of the interface is what geometers call motion by mean curvature: the velocity of the hypersurface is normal and proportional to the mean curvature. In other words, the global picture is essentially a dynamical Plateau Problem [Bra].

The evolution problem for $\Gamma^{0}(s)$, specified by equations $(1.13),(1.15)$ is known to have a unique local solution, i.e., up to $s<T_{\text {sing }}$, where $T_{\text {sing }}>0$.

Our approach to the problem consists in providing a sound mathematical foundation to a Chapman-Enskog expansion, corresponding to the intuition often put forward by physicists, that the leading term in the expansion should describe the evolution of the interface by motion along the gradient of the surface tension [AlCa, $\mathrm{KaOh}, \mathrm{Sp}$ ].

In this article, we give an asymptotic expansion of arbitrary large order for the solution of (1.2) with initial condition of the form

$$
u_{0}(x)=\boldsymbol{\theta}\left(\frac{\Lambda^{0}(x, 0)}{h}\right)
$$

in the neighbourhood of $\Gamma_{0}=\left\{\Lambda^{0}(x, 0)=0\right\}$, which is supposed to be a $N-1$ dimensional compact manifold. The expansion is valid for times $t \leq h^{-2} T^{*}$, where $T^{*}$ is any positive number less than $T_{\text {sing }}$. The asymptotic expansion of $u$ is of the form

$$
u(x, t ; h) \sim \sum_{j \geq 0} v^{j}\left(\frac{\Lambda\left(x, h^{2} t ; h\right)}{h}, \Sigma\left(x, h^{2} t ; h\right), t, h^{2} t\right) h^{j},
$$

and the asymptotic expansion of the distance function to the interface is

$$
\Lambda(x, s ; h) \sim \sum_{j \geq 0} \Lambda^{j}(x, s) h^{j}
$$

Here $\Sigma\left(x, h^{2} t ; h\right)$ describes tangential coordinates.

The expansion technique is fairly obvious: for all $j$ we equate to zero the coefficients of $h^{j}$ in the expansion of the equation. The lowest order term $v^{0}$ will be equal to $\boldsymbol{\theta}(\mu)$. The second term $v^{1}$ stays bounded for all time if we require an orthogonality condition. This condition is exactly (1.15). It is a fortunate circumstance that $v^{1}$ vanishes, but $v^{2}$ does not, as long as $\Gamma^{0}(s)$ is not flat (see the end of $\S 3$ ). So, in contrast to the one-dimensional case, the exact solution cannot be closer to $v^{0}$ than an $O\left(h^{2}\right)$ in $L^{\infty}$ norm. At higher $\operatorname{order}(j \geq 1)$, each $v^{j}$ satisfies an equation of the form

$$
\frac{\partial v^{j}}{\partial t}+A v^{j}=-P^{j}
$$

with a source term $P^{j}$ depending on $v^{k}$ and its derivatives $(k \leq j-2)$, and on $\Lambda^{k}$ and its derivatives $(k \leq j-1)$. The $v^{j}$ 's stay bounded for all time if the source term is orthogonal for large $t$ 's to the kernel of the operator $A$. This leads to an equation for $\Lambda^{j}$ of the form

$$
\frac{\partial \Lambda^{j}}{\partial s}-\Delta^{\Gamma^{0}(s)} \Lambda^{j}-C(\sigma, s) \Lambda^{j}=Q_{0}^{j}
$$


where $\Delta^{\Gamma^{0}(s)}$ is the Laplace operator of the manifold $\Gamma^{0}(s)$, equipped with the metric induced by $\mathbb{R}^{N}, C(\sigma, s)$ is the sum of the squares of the scalar curvatures, and $Q_{0}^{j}$ depends only on $\Lambda^{k}(k \leq j-2)$, and $\lim _{t \rightarrow+\infty} v^{k}(k \leq$ $j-2)(\S 3)$.

All the terms in the asymptotic expansion are estimated in a number of functional spaces. In particular we show that for $j \geq 1$ the quantity $\left|v^{j}(\mu, \sigma, s, t)\right|$ converges exponentially to zero as $\mu$ tends to infinity, uniformly in $\sigma, s, t$. This enables us to extend the expansion (1.17) defined on a tubular neighbourhood of $\Gamma^{0}(s)$ as a $C^{\infty}$ function on $\mathbb{R}^{N}$. Then we estimate in $L^{2}$ and $L^{\infty}$ the remainder $\rho^{q, h}=\left(\partial / \partial t-h^{2} \Delta\right) u^{q, h}+\phi\left(u^{q, h}\right)$ where $u^{q, h}$ is the expansion truncated at $q$ terms $(\S 4)$. We have to estimate the derivatives of any order in $s$ and $\sigma$ because we perform an inductive proof which involves more and more differentiations.

The estimates on the remainder enable to appreciate the error made when taking the truncated expansion in place of the true solution. The idea of the error estimate is the following: $v$ be the exact solution of (1.2) with initial data (1.3). The difference $w=v-u^{q, h}$ satisfies

$$
\frac{\partial w}{\partial t}-h^{2} \Delta w+\phi^{\prime}\left(u^{q, h}\right)=-\rho^{q, h}-\psi\left(u^{q, h}, w\right) w^{2} .
$$

The remainder $\rho^{q, h}$ is small if we take $q$ large enough, and $\psi$ is bounded when its arguments are bounded. Estimates in $L^{2}$ do not work because of the nonlinear term on the right hand side. Therefore, we combine $L^{2}$ and $L^{\infty}$ estimates in a bootstrap procedure, using connectedness in time. We provide error bounds in $L^{2}$ and $L^{\infty}$ of the whole space ( $\left.\S 6\right)$. It turns out that the number of terms $q$ we need to estimate the error increases linearly with the space dimension $N$. This is why we need expansions of arbitrary order.

We can perform the $L^{2}$ estimates because the spectrum of the selfadjoint operator $-h^{2} \Delta+\phi^{\prime}\left(u^{q, h}\right)$ can be estimated from below by $-C h^{2}$. The potential $\phi^{\prime}\left(u^{q, h}\right)$ is everywhere strictly positive except in a region of width $O(h)$ around the interface. Therefore, all the interesting phenomena happen very close to the interface. This rough picture can be made precise by showing that if $\zeta^{h}$ is the normalized eigenvector corresponding to the smallest eigenvalue, then

$$
\int_{\mathscr{V}[\sqrt{h}]^{c}}\left(h^{2}|\nabla \zeta|^{2}+|\zeta|^{2}\right) d x \leq C^{\prime} e^{-C / \sqrt{h}} .
$$

Here $\mathscr{V}[\sqrt{h}]$ is the set of points located at a distance less than $\sqrt{h}$ from the interface. Hence we may reduce ourselves to a Neumann problem in a tubular neighbourhood of the interface of width $O(\sqrt{h})$. As we are interested in a lower bound, we consider only the normal modes, which leads us to a collection of Neumann problems depending on the tangential variable $\sigma$ as a parameter. We deal with them by comparing them to a $\sigma$-independent Neumann problem, and by using precise perturbation techniques $(\S 5)$.

The symmetry assumption we made on the potential $\Phi$ was introduced only for convenience. We believe that all the results proved here go through for more general potentials, the only essential requirement being that $\Phi$ has two wells of equal depth.

The compactness assumption on $\Gamma_{0}$ is technical in nature. It could be replaced by suitable uniformity assumptions. 
Our analysis can be applied to the one-dimensional case: then all terms of the asymptotic expansion are zero, except the term of order zero ; the same situation, intuitively characterized by the absence of surface tension, prevails in case of a flat interface. Using the same strategy as here, with less geometry and more subtle estimates,since we must deal with exponentially small terms the results of [CaPe, FuHa and $\mathrm{Fu}] \mathrm{can}$ be recovered and made more precise, as we will show in a forthcoming article; see subsection 1.3 for more detailed bibliographic information.

The results we present here have been announced in [MoSc1].

1.2. Motion by mean curvature. Let $\bar{\Gamma}$ be a fixed reference manifold, and let $\sigma \mapsto X^{0}(0, \sigma, s)$ be a diffeomorphism between $\bar{\Gamma}$ and $\Gamma^{0}(s)$. If $\nu(\sigma, s)$ is the exterior normal to $\Gamma^{0}(s)$ at $X^{0}(0, \sigma, s)$, we define

$$
X^{0}(\lambda, \sigma, s)=X^{0}(0, \sigma, s)+\lambda \nu(\sigma, s) .
$$

The mapping $X^{0}(., ., s)$ is a diffeomorphism from $[-\bar{\lambda}, \bar{\lambda}] \times \bar{\Gamma}$ to a tubular neighbourhood $\mathscr{V}^{0}(s)$ of $\Gamma^{0}(s)$. The inverse diffeomorphism is $x \mapsto$ $\left(\Lambda^{0}(x, s), \Sigma^{0}(x, s)\right)$. This can be expressed as

$$
x=X^{0}\left(\Lambda^{0}(x, s) \Sigma^{0}(x, s), s\right), \quad \forall x \in \mathscr{V}^{0}(s) .
$$

Without loss of generality, we may assume that

$$
\frac{\partial X^{0}}{\partial s}(0, \sigma, s) \text { is parallel to } \nabla \Lambda^{0}\left(X^{0}(0, \sigma, s)\right), \quad \forall \sigma, s .
$$

This assumptions freezes the parametrization of $\Gamma^{0}(s)$ by $\bar{\Gamma}$, up to a reparametrization of $\bar{\Gamma}$ which does not depend on time (Lemma 3.3). If we differentiate (1.19) with respect to $s$, we obtain

$$
\begin{aligned}
0= & \frac{\partial X^{0}}{\partial \lambda}\left(\Lambda^{0}(x, s), \Sigma^{0}(x, s), s\right) \frac{\partial \Lambda}{\partial s}(x, s) \\
& +\frac{\partial \Sigma^{0}}{\partial s}(x, s) \cdot X^{0}\left(\Lambda^{0}(x, s), \Sigma^{0}(x, s), s\right) \\
& +\frac{\partial X^{0}}{\partial s}\left(\Lambda^{0}(x, s), \Sigma^{0}(x, s), s\right) .
\end{aligned}
$$

Here $\left(\partial \Sigma^{0} / \partial s\right) \cdot X^{0}$ denotes the action of the vector field $\partial \Sigma^{0} / \partial s$ on $X^{0}$ (differentiation of $X^{0}$ in the direction of $\partial \Sigma^{0} / \partial s$ ). This vector field notation will be repeatedly used throughout the paper.

Relation (1.18) implies that

$$
\frac{\partial X^{0}}{\partial \lambda}\left(\Lambda^{0}(x, s), \Sigma^{0}(x, s), s\right)=\nu\left(\Sigma^{0}(x, s), s\right), \quad \forall x \in \Gamma^{0}(s) .
$$

Relation (1.21) becomes, by virtue of (1.20), (1.22) and the equation for $\Lambda^{0}$ (1.15)

$$
\frac{\partial X^{0}}{\partial s}\left(\Lambda^{0}(x, s), \Sigma^{0}(x, s), s\right)=-\nu(x, s) \Delta \Lambda^{0}(x, s), \quad \forall x \in \Gamma^{0}(s) .
$$

A calculation in local coordinates shows that $\Delta \Lambda^{0}(x, s)$ is the mean curvature of $\Gamma^{0}(s)$, i.e., the sum of the scalar curvatures. The sign convention is that the mean curvature of a convex hypersurface is positive. Thus (1.23) is precisely the motion by mean curvature. 
1.3. Bibliographical remarks. Equation (1.2) has been proposed to describe the dynamics of various alloys such as $\mathrm{Fe}_{3} \mathrm{Al}, \mathrm{Ni}_{3} \mathrm{Mn}$ and $\mathrm{CuZn}$ which undergo an order-disorder transition. In such a model, the order parameter is not conserved. Such interfaces are called antiphase boundaries in the physical literature; the theory of antiphase boundary motion has been extensively studied by Allen and Cahn. They predicted that the motion of a gently curved interface is proportional to its mean curvature [AlCa]. The dynamics of first order phase transitions is a very active subject in physics. The reader is referred to the extensive review [GuSMSa] for an introduction to the physicists' point of view.

The stationary problem for (1.2), possibly coupled with a mass constraint, has been studied in [Gu, KoSt, GuMa, Mo, LuMo and CaGuSl]. The evolution problem in one dimension has been tackled by [Ne], and has been developed by [CaPe, FuHa, Fu, BroKo1]. L. Bronsard and R. V. Kohn [Bro, BroKo2] worked out the $N$-dimensional case under the assumption of radial symmetry; their methods are very different from ours. J. Rubinstein, P. Sternberg and J. Keller [RuStKe] have obtained by formal asymptotics the lowest order term of the expansion in two dimensional space, and moreover they consider the interaction with boundaries. Related results have been obtained independently by Danilov and Subochev [DaSu]. A probabilistic approach was used by Freidlin [Fre], Presutti [Pr], Benzi, Jona-Lasinio, Sutera [BeJoSu].

Related papers are [KaOh, $\mathrm{CgFi}, \mathrm{Cg}, \mathrm{Fi}, \mathrm{FiGi}, \mathrm{FiHs}, \mathrm{AnGu}, \mathrm{An} 1, \mathrm{An} 2, \mathrm{An} 3$, No].

Concerning the motion by mean curvature (see subsection 1.2), Hamilton [Ha] has proved by Nash-Moser estimates that this problem has a unique solution locally in time; DeTurck [Dt] has reduced it to a nonlinear integrodifferential parabolic problem, Brakke [Bra] treats dynamical Plateau problems in the frame of geometrical measure theory, and a number of articles consider more specialized questions [ $\mathrm{Gr}, \mathrm{GaHa}, \mathrm{Hu}$ ].

After the present research project was completed, Chen [Ch] found a simpler and shorter proof of the fact that interfaces move by mean curvature ; his proof relies heavily on the maximum principle and works before singularities appear. In a forthcoming paper [ESS], Evans, Soner and Souganidis show that interfaces move by mean curvature, even beyond the time of appearance of singularities. Once again their proof relies on the maximum principle, since it uses techniques of viscosity solutions for Hamilton-Jacobi equations, according to P. L. Lions and M.G. Crandall. In both papers, the authors show that at a distance $o(1)$ from the moving interface, the solution is very close to \pm 1 , but they do not obtain a profile for the solutions as we do.

It cannot be expected that proofs relying on the maximum principle can be generalized for higher order problems such as Cahn-Hilliard equation; the maximum principle is used in this paper only for time dependent estimates, and it could probably be substituted by some other technique. The spectral information (1.8) on the one-dimensional problem is highly dependent on the maximum principle. However, $\S 5$ of this paper uses only Rayleigh quotients and hilbertian estimates.

The news of the untimely and tragic death of $P$. de Mottoni on November 25,1990 reached the second author while this article was in the last steps of the revision process. 


\section{EXISTENCE AND UNIQUENESS; ESTIMATES}

Let $\phi$ be a smooth function from $\mathbb{R}$ to itself; assume that there exists $R>0$ such that

$$
|r| \geq R \Rightarrow r \phi(r) \geq 0 .
$$

In this section, we prove the following existence and uniqueness result:

Proposition 2.1. For any $u_{0}$ in $L^{\infty}\left(\mathbb{R}^{N}\right)$ and any $h>0$, there exists a unique function $u$ belonging to $L^{\infty}\left(\mathbb{R}^{N} \times[0, T]\right)$ for all $T>0$, continuous from $\mathbb{R}^{+}$ to $\mathscr{D}^{\prime}\left(\mathbb{R}^{N}\right)$ that satisfies

$$
u_{t}-h^{2} \Delta u+\phi(u)=0 \text { in the sense of distributions on } \mathbb{R}^{N} \times \mathbb{R}^{+} ;
$$

and the initial condition

$$
u(x, 0)=u_{0}(x) .
$$

Moreover such a function $u$ is infinitely differentiable over $\mathbb{R}^{N} \times(0, \infty)$, and the following estimate holds

$$
|u(., t)|_{\infty} \leq \max \left(\left|u_{0}\right|_{\infty}, R\right) .
$$

This result is not surprising at all ; one might think that it is a standard consequence of the large body of results pertaining to semilinear problems which are bounded perturbations of monotone problems; unfortunately, theorems which cover the case of an unbounded domain and $L^{\infty}$ initial data do not seem to be available; we could have started from existing results and performed an approximation procedure, but it did not shorten the proof; moreover, we need the global estimates of subsection 2.3 below. The proof is organized as follows: in subsection 2.1, we prove local estimates and regularity; in subsection 2.2, we apply the maximum principle to obtain the uniqueness, and in subsection 2.3, we obtain uniform bounds on the maximum norm of $u(., t)$ and all its derivatives on $\mathbb{R}^{N} \times[T,+\infty]$, for all $T>0$. The proof is concluded in subsection 2.4.

2.1. Local estimates. The first estimates we prove are local in time; assume that (2.2) has a solution satisfying the initial condition, and essentially bounded over $\mathbb{R}^{N} \times[0, T]$ for all positive $T$. Let $f=-\phi(u)$. We denote by $|v|_{\infty}$ the norm of an element of $L^{\infty}\left(\mathbb{R}^{N} \times[0, T]\right)$. Under our assumptions, the function $f$ belongs to $L^{\infty}\left(\mathbb{R}^{N} \times[0, T]\right)$.

Let $\mathscr{E}$ denote the fundamental solution of the heat equation:

$$
\mathscr{C}_{t}-\Delta \mathscr{E}=\delta \quad \text { in } \mathbb{R}^{N} \times \mathbb{R}, \quad \text { supp } \mathscr{E} \subset \mathbb{R}^{N} \times \mathbb{R}^{+} ;
$$

it is given explicitly by

$$
\mathscr{E}(x, t)=(4 \pi t)^{-N / 2} \exp \left(-|x|^{2} / 4 t\right) .
$$

The following relation is a standard consequence of the properties of fundamental solutions: for $t^{\prime}<t$,

$$
u(., t)=\mathscr{E}\left(., h^{2}\left(t-t^{\prime}\right)\right) * u\left(., t^{\prime}\right)+\int_{t^{\prime}}^{t} \mathscr{E}\left(., h^{2}(t-s)\right) * f(., s) d s
$$


In order to estimate the spatial derivatives of $u$, we apply the method of translations; we define finite difference operators $\nabla_{k}^{\theta}$ for $\theta \neq 0$ by

$$
\left(\nabla_{k}^{\theta} v\right)(x)=\frac{v\left(x+\theta e_{k}\right)-v(x)}{\theta} .
$$

where $\left(e_{1}, \ldots e_{N}\right)$ is the canonical basis of $\mathbb{R}^{N}$. If we apply $\nabla_{k}^{\theta}$ to $(2.6)$ with $t^{\prime}=0$, we obtain

$$
\nabla_{k}^{\theta} u(., t)=\left(\nabla_{k}^{\theta} \mathscr{E}\left(., h^{2} t\right)\right) * u_{0}+\int_{0}^{t} \nabla_{k}^{\theta} \mathscr{E}\left(., h^{2}(t-s)\right) * f(., s) d s .
$$

An elementary computation shows that for all $t>0$,

$$
\int\left|\frac{\partial \mathscr{E}}{\partial x_{k}}\left(x, h^{2} t\right)\right| d x \leq \frac{C_{1}}{h \sqrt{t}} .
$$

Therefore,

$$
\int\left|\nabla_{k}^{\theta} \mathscr{E}\left(., h^{2} t\right)\right| d x \leq \frac{C_{1}}{h \sqrt{t}} .
$$

With the help of (2.8), we obtain from from (2.7)

$$
\left|\nabla_{k}^{\theta} u(., t)\right|_{\infty} \leq \frac{C_{1}}{h \sqrt{t}}\left|u_{0}\right|_{\infty}+\frac{2 C_{1} \sqrt{t}}{h}|f|_{\infty} .
$$

Therefore, for all times $T_{1}$ such that $0<T_{1}<T<\infty, u$ is Lipschitz continuous in space on $\mathbb{R}^{N} \times\left[T_{1}, T\right]$, and its spatial derivatives satisfy

$$
\left|\frac{\partial u}{\partial x_{k}}\right|_{\infty} \leq h^{-1} L_{1}\left(T_{1}, T,|u|_{\infty}\right) \text {. }
$$

If we apply two finite difference operators to (2.6) with $t^{\prime}=T_{1}$ we shall have

$$
\begin{aligned}
& \nabla_{k}^{\theta} \nabla_{j}^{\eta} u(., t)=\left[\nabla_{k}^{\theta} \mathscr{E}\left(., h^{2}\left(t-T_{1}\right)\right)\right] *\left[\nabla_{j}^{\eta} u\right]\left(., T_{1}\right) \\
& +\int_{T_{1}}^{t}\left[\nabla_{k}^{\theta} \mathscr{E}\left(., h^{2}(t-s)\right)\right] *\left[\nabla_{j}^{\eta} f(., s)\right] d s .
\end{aligned}
$$

From (2.9), we deduce that

$$
\left|\nabla_{j}^{\eta} f\right|_{\infty} \leq\left|\nabla_{j}^{\eta} u\right|_{\infty} \max \left\{\left|\phi^{\prime}(r)\right| / r \leq|u|_{\infty}\right\} \leq h^{-1} L_{1} \max \left\{\left|\phi^{\prime}(r)\right| / r \leq|u|_{\infty}\right\},
$$

uniformly in $\eta$ and $j$. Therefore, we obtain the estimate

$$
\left|\nabla_{k}^{\theta} \nabla_{j}^{\eta} u(., t)\right| \leq \frac{C_{1} L_{1}}{h^{2}}\left[\frac{1}{\sqrt{t-T_{1}}}+2 \max \left\{\left|\phi^{\prime}(r)\right| / r \leq|u|_{\infty}\right\} \sqrt{t-T_{1}}\right] .
$$

Therefore, for all $T_{2} \in\left(T_{1}, T\right)$, the finite differences $\nabla_{k}^{\theta} u$ are uniformly Lipschitz continuous; by Ascoli-Arzelà theorem, $u$ is continuously differentiable, and its first derivatives are Lipschitz continuous, with Lipschitz constant $h^{-2} L_{2}$ :

$$
L_{2} \leq C_{1} L_{1}\left[\frac{1}{\sqrt{T_{2}-T_{1}}}+2 \max \left\{\left|\phi^{\prime}(r)\right| / r \leq|u|_{\infty}\right\} \sqrt{T-T_{1}}\right] .
$$

This enables us to differentiate (2.6) with respect to $x_{j}$ at time $t^{\prime}=T_{2}$ :

$\partial_{j} u(x, t)=\mathscr{E}\left(., h^{2}\left(t-T_{2}\right)\right) * \partial_{j} u\left(., T_{2}\right)+\int_{T_{2}}^{t} \mathscr{E}\left(., h^{2}(t-s)\right) *\left(\phi^{\prime}(u) \partial_{j} u\right)(., s) d s$. 
An inductive argument, whose details are left to the reader, shows that for $\alpha=\left(\alpha_{1}, \ldots, \alpha_{N}\right)$ a multi-index, $\partial^{\alpha}=\partial_{1}^{\alpha_{1}} \cdots \partial_{N}^{\alpha_{N}},|\alpha|=\alpha_{1}+\cdots+\alpha_{N}$,

$$
\begin{aligned}
\partial^{\alpha} u(x, t)= & \mathscr{E}\left(., h^{2}\left(t-T_{|\alpha|}\right)\right) * \partial^{\alpha} u\left(., T_{|\alpha|}\right) \\
& +\int_{T_{|\alpha|}}^{t} \mathscr{E}\left(., h^{2}(t-s)\right) * F_{\alpha}\left(\partial^{\beta} u, \beta \leq \alpha\right)(., s) d s
\end{aligned}
$$

here $F_{\alpha}$ is a smooth function depending on the arguments $\partial^{\beta} u$, for $\beta_{k} \leq$ $\alpha_{k}, 1 \leq k \leq N$, and the sequence $T_{n}$ is increasing. On each strip $\mathbb{R}^{N} \times$ $\left[T_{n}, T\right]$, we have the estimate, for $|\alpha|=n$

$$
\left|\partial^{\alpha} u(., t)\right|_{\infty} \leq h^{-n} L_{n}\left(T_{1}, T_{2}, \ldots, T_{n}, T,|u|_{\infty}, \phi\right) .
$$

The constant $L_{n}$ depends on $\phi$ through the bounds over $\phi$ and its derivatives of order at most $n$ over the interval $\left[-|u|_{\infty},|u|_{\infty}\right]$. Therefore, we have the desired regularity, with the additional information (2.11).

2.2. Uniqueness. In order to obtain the uniqueness, we consider two solutions $u$ and $v$ of (2.2) with respective initial conditions $u_{0}$ and $v_{0}$. Let

$$
M(t)=\max (0, \operatorname{ess} \sup (u(., t)-v(., t))) .
$$

Denote

$$
\gamma=\sup \left\{\left|\phi^{\prime}(r)\right| /|r| \leq \max \left(|u|_{\infty},|v|_{\infty}\right)\right\} .
$$

Assume that for some $t>0$ there is a point $x_{t}$ such that

$$
M(t)=u\left(x_{t}, t\right)-v\left(x_{t}, t\right)>0 .
$$

then

$$
\Delta(u-v)\left(x_{t}, t\right) \leq 0
$$

and as $M$ is left differentiable over $(0, T)$,

$$
\frac{\partial(u-v)}{\partial t}\left(x_{t}, t\right) \geq \frac{d^{-}}{d t} M(t),
$$

for all $t>0$. Thus,

$$
\frac{d^{-}}{d t} M(t) \leq-\phi^{\prime}(w) M(t)
$$

where

$$
w=w(x, t) \in[\min (u(x, t), v(x, t)), \max (u(x, t), v(x, t))] .
$$

This implies that

$$
\frac{d^{-}}{d t} M(t) \leq \gamma M(t) .
$$

Assume now that there is no point such that (2.12) holds. If $M(t)>0$, there is a sequence $\left\{x_{j}\right\}$, with $\left|x_{j}\right| \rightarrow \infty$ as $j \rightarrow \infty$ such that

$$
\lim _{j \rightarrow \infty}\left(u\left(x_{j}, t\right)-v\left(x_{j}, t\right)\right)=M(t) .
$$

An elementary argument shows that

$$
\lim _{j \rightarrow \infty}\left(\nabla u\left(x_{j}, t\right)-\nabla v\left(x_{j}, t\right)\right)=0,
$$




$$
\limsup _{j \rightarrow \infty}\left(\Delta u\left(x_{j}, t\right)-\Delta v\left(x_{j}, t\right)\right) \leq 0 .
$$

Therefore, we still have (2.13). If $M(t)=0$, and there is no point such that (2.12) holds, $(2.13)$ is still true: one has to consider the two cases: $\sup (u(., t)-$ $v(., t))=0$, as previously and $\sup (u(., t)-i v(., t))<0$, which is trivial. Let us show now that

$$
\limsup _{t \rightarrow 0} M(t)=M(0) \text {. }
$$

As $u(., t)$ is bounded in $L^{\infty}\left(\mathbb{R}^{N}\right)$ uniformly in $t \in[0, T]$, and converges to $u_{0}$ in the sense of distributions as $t$ tends to zero, we can see that $u(., t) \rightarrow u_{0}$ in $L^{\infty}\left(\mathbb{R}^{N}\right)$ weak-*. In particular, for $t \leq s$,

$$
|u(., t)-v(., t)|_{\infty} \leq \sup _{[0, s]} M(t),
$$

and therefore,

$$
\left|u_{0}-v_{0}\right|_{\infty} \leq \inf _{s \in[0, T]} \sup _{t \in[0, s]} M(t)=\limsup _{s \rightarrow 0} M(s) .
$$

On the other hand, (2.6) implies that

$$
|u(., t)-v(., t)|_{\infty} \leq\left|u_{0}-v_{0}\right|_{\infty}+o(1) .
$$

Hence we obtain (2.14). From (2.13), we deduce that $M(t) \leq M(0) e^{\gamma t}$. In particular, the following implication holds:

$$
u_{0} \leq v_{0} \text { a.e. } \Rightarrow u(x, t) \leq v(x, t), \quad \forall(x, t) \in \mathbb{R}^{N} \times(0, \infty) .
$$

Exchanging the roles of $u$ and $v$, we can conclude that

$$
|(u-v)(., t)|_{\infty} \leq e^{\gamma t}\left|u_{0}-v_{0}\right|_{\infty}
$$

We obtained a quantitative estimate of the continuous dependence of the solution with respect to the data; it implies in particular the uniqueness.

2.3. Global estimates. Consider a particular solution of (2.2) defined by

$$
v(x, t)=\rho(t) ; \quad \rho(0)=\max \left(R,\left|u_{0}\right|_{\infty}\right) ; \quad \rho^{\prime}(t)+\phi(\rho(t))=0 .
$$

Thanks to assumption (2.1), $\rho$ is defined for all $t \geq 0$, and satisfies the estimate

$$
|\rho(t)| \leq|\rho(0)|, \quad \forall t \geq 0 .
$$

Moreover, $v$ is a solution of (2.2) with initial condition $v_{0}=\rho(0)$. Therefore, if $u$ is a solution of (2.2), it satisfies (2.4). For all the derivatives of $u$ we shall obtain estimates holding uniformly away from $t=0$ : assume that we have found $T_{1}<T_{2}<\cdots<T_{n}$ such that

$$
\forall m \leq n, \forall \alpha \text { such that }|\alpha|=m, \forall t \geq T_{m}:\left|\partial^{\alpha} u(., t)\right|_{\infty} \leq K_{m} h^{-m} .
$$

Let

$$
c_{n}=\max _{|\alpha|=n} \sup _{t \geq T_{n}}\left|F_{\alpha}\left(\partial^{\beta} u, \beta \leq \alpha\right)(., t)\right|_{\infty},
$$

which is bounded according to the induction hypothesis; according to (2.10), we can write the inequality, for all $t, t^{\prime}$ such that $T_{n} \leq t^{\prime}<t$, and $\alpha^{\prime}$ with $\left|\alpha^{\prime}\right|=n+1$ :

$$
\left|\partial^{\alpha^{\prime}} u(., t)\right|_{\infty} \leq \frac{C_{1}}{h^{n+1}}\left[\frac{K_{n}}{\sqrt{t-t^{\prime}}}+2 c_{n} \sqrt{t-t^{\prime}}\right]
$$


If $\tau_{n}$ is some strictly positive number, the choice $T_{n} \leq t^{\prime}$ and $t=t^{\prime}+\tau_{n}$ implies that for $t \geq T_{n+1}=T_{n}+\tau_{n}$

$$
\left|\partial^{\alpha^{\prime}} u(., t)\right|_{\infty} \leq h^{-(n+1)} K_{n+1},
$$

with

$$
K_{n+1}=C_{1}\left[\frac{K_{n}}{\sqrt{\tau_{n}}}+2 c_{n} \sqrt{\tau_{n}}\right] .
$$

2.4. Existence. Given $u_{0}$ in $L^{\infty}\left(\mathbb{R}^{N}\right), H>0, \rho(0)$ as in (2.17), and $\omega$ an infinitely differentiable function with compact support such that

$$
\begin{cases}\omega(r)=1 & \text { if }|r| \leq \rho(0)+1 \\ \omega(r)=0 & \text { if }|r| \geq \rho(0)+2 \\ \omega(r) \in[0,1] & \text { otherwise }\end{cases}
$$

we define $\tilde{\phi}$ by

$$
\tilde{\phi}(r)=\omega(r) \phi(r)+(1-\omega(r)) H|r| .
$$

Therefore, $\tilde{\phi}$ is smooth, uniformly Lipschitz continuous on $\mathbb{R}$ and satisfies condition (2.1). Solutions of $(2.2),(2.3)$ with $\phi$ replaced by $\tilde{\phi}$ are fixed points of the operator

$$
\mathscr{T} u(., t)=\mathscr{E}\left(., h^{2} t\right) * u_{0}-\int_{0}^{t} \mathscr{E}\left(., h^{2}(t-s)\right) * \tilde{\phi}(u(., s)) d s .
$$

By Picard iterations, we can find for all positive $T$ an integer $p \in \mathbb{N}_{*}$ such that $\mathscr{T}^{p}$ is strictly contracting in $L^{\infty}\left(\mathbb{R}^{N} \times[0, T]\right)$. Thus, $\mathscr{T}$ has a unique fixed point; according to Step 3, this fixed point satisfies $|u(., t)|_{\infty} \leq \rho(0)$. As $\phi$ and $\tilde{\phi}$ coincide over $[-\rho(0), \rho(0)]$, the existence is proved.

\section{ASYMPTOTIC EXPANSION}

Let $\Gamma_{0}$ be a compact smooth manifold of dimension $N-1$ embedded in $\mathbb{R}^{N}$, and let $\Lambda_{0}(x)$ be the normal coordinate of $x$ with respect to $\Gamma_{0}$. In a small enough neighborhood of $\Gamma_{0}, \Lambda_{0}$ is a smooth function of $x$. In this section we prove that problem (2.2) with initial data of the form

$$
u_{0}(x)=\Theta\left(\frac{\Lambda_{0}(x)}{h}\right) \text { in a neighbourhood of } \Lambda_{0}
$$

possesses an asymptotic expansion of arbitrary order.

In the first subsection, we establish some technical lemmas; next, we introduce the reader to a few geometric facts that are needed to write intrinsic operators on manifolds and prove that the knowledge of the normal coordinate $\Lambda(x, s ; h)$ to the interface $\Gamma(s ; h)$, coupled with a very natural geometric normalization condition, determines the parametrization $X(\lambda, \sigma, s ; h)$ of the interface up to a reprametrization which is independent of $s$ and $h$. In subsection 3.3 we shall formally set up the asymptotic expansion. This involves as well an expansion for the parametrization $X(\lambda, \sigma, s ; h)$ of the interface $\Gamma(s ; h):$ we shall prove that the normalization condition exploited in subsection 3.2 permits to determine, at every step of the expansion, the parametrization of $\Gamma$ in terms of the normal coordinate $\Lambda:$ in other words, at every step of the expansion, the contribution to the tangential coordinates is fully determined. The complete algorithm for 
the construction of the successive terms of the development will be described in subsections 3.4 (devoted to the equations for the $\Lambda^{j}$ 's) and 3.5 (devoted to the equations for the $v^{j}$ 's). This algorithm will be justified rigorously by the estimates of $\S 4$.

3.1. Generalized chain rules. The following technical lemmas will enable us to make asymptotic expansions of composed functions:

For $k \in \mathbb{N}$, let us define the set

$$
A_{k}=\left\{\alpha \in(\mathbb{N})^{\mathbb{N}}, \alpha_{j}=0 \text { for } j \text { large enough } / \sum_{j \geq 0} j \alpha_{j}=k\right\} .
$$

Lemma 3.1. Let $g: \mathbb{R} \mapsto \mathbb{R}^{N}, f: \mathbb{R}^{N} \mapsto \mathbb{R}$ be $C^{\infty}$ functions. Then for any $k \in \mathbb{N}$ :

$$
\frac{d^{k}}{d x^{k}}(f \circ g)(x)=\sum_{\alpha \in A_{k}} a_{\alpha}^{k} D^{|\alpha|} f(g(x)) \bigotimes_{j=1}^{k}\left(g^{(j)}(x)\right)^{\otimes \alpha_{j}}
$$

where $|\alpha|=\sum \alpha_{j}, D^{|\alpha|} f$ denotes the differential of $f$ of order $|\alpha|$, and $a_{\alpha}^{k}$ are positive numbers.

Proof. The result is obvious if $k=1$. Suppose it holds for $k \leq l$ : to show that it holds for $k=l+1$, consider

$$
\begin{aligned}
\frac{d}{d x}\left(D^{|\alpha|} f(g(x)) \bigotimes_{j=1}^{l}\left(g^{(j)}(x)\right)^{\otimes \alpha_{j}}\right) \\
\quad=\left\{D^{|\alpha|+1} f(g(x)) \bigotimes_{j=1}^{l}\left(g^{(j)}(x)\right)^{\otimes \beta_{j}^{\circ}}+D^{|\alpha|} f(g(x)) \sum_{s=1}^{l} \alpha_{s} \bigotimes_{j=1}^{l+1}\left(g^{(j)}(x)\right)^{\beta_{j}^{s}}\right\}
\end{aligned}
$$

where

$$
\begin{aligned}
& \beta_{j}^{0}=\left(\alpha_{1}+1, \alpha_{2}, \ldots, \alpha_{l}\right), \\
& \beta_{j}^{s}=\left(\alpha_{1}, \ldots, \alpha_{s-1}, \alpha_{s}-1, \alpha_{s+1}+1, \ldots, \alpha_{l}\right), \text { for } 1 \leq s<l, \\
& \beta_{j}^{l}=\left(\alpha_{1}, \alpha_{2}, \ldots, \alpha_{s-1}, 1\right) .
\end{aligned}
$$

A straightforward calculation provides

$$
\left|\beta^{0}\right|=l+1, \quad\left|\beta^{l}\right|=l, \quad 1 \leq s \leq l ;
$$

moreover

$$
\begin{aligned}
\sum_{j \geq 1} j \beta_{j}^{0} & =\alpha_{1}+1+\sum_{j \geq 2} j \alpha_{j}=l+1 \\
\sum_{j \geq 1} j \beta_{j}^{s} & =\sum_{1 \leq j \leq s-1} j \alpha_{j}+s \alpha_{s}-s+(s+1) \alpha_{s+1}+(s+1)+\sum_{j \geq s+2} j \alpha_{j} \\
& =\sum_{j \geq 1} j \alpha_{j}+1=l+1 \quad \text { for } 1 \geq s \geq k-1 \\
\sum_{j \geq 1} j \beta_{j}^{l} & =l+1
\end{aligned}
$$


The new coefficients are still positive numbers: in view of (3.3), (3.4) we conclude that (3.1) holds for $k=l+1$ as well.

Lemma 3.2. Let $g: \mathbb{R} \mapsto \mathbb{R}^{N}, f: \mathbb{R} \times \mathbb{R}^{N} \mapsto \mathbb{R}$ be $C^{\infty}$ functions. Then

$$
\begin{aligned}
& \frac{d^{k}}{d x^{k}}\{f(x, g(x))\} \\
& \quad=\frac{\partial^{k} f}{\partial x_{1}^{k}}(x, g(x))+\sum_{p=1}^{k} C_{k}^{p} \sum_{\alpha \in A_{p}} a_{\alpha}^{p} \frac{\partial^{k-p}}{\partial x_{1}^{k-p}} D_{x_{2}}^{|\alpha|} f(x, g(x)) \bigotimes_{j=1}^{p}\left(g^{(j)}(x)\right)^{\otimes \alpha_{j}}
\end{aligned}
$$

Proof. Let $f(x, g(y))=h(x, y)$.

It is easy to show that

$$
\frac{d^{k}}{d x^{k}} h(x, x)=\sum_{p=1}^{k} C_{k}^{p} \frac{\partial^{k} h(x, x)}{\partial x_{1}^{k-p} \partial x_{2}^{p}}+\frac{\partial^{k} f}{\partial x_{1}^{k}}
$$

The result follows by virtue of Lemma 3.1. As an application of Lemma 3.1, take $\phi \in C^{\infty}, 1 \leq k$, and define

$$
\tilde{\Psi}^{k}\left(v^{0}, \ldots, v^{k-1}\right)=\sum_{\substack{\alpha \in A_{k} \\ \alpha \neq(0, \ldots, 0,1)}} a_{\alpha}^{k} \phi^{(|\alpha|)}\left(v^{0}\right) \prod_{j=1}^{k}\left(j ! v^{j}\right)^{\alpha_{j}}
$$

Then we can write, for any $q \geq k$

$$
\left.k ! \frac{d^{k}}{d h^{k}} \phi\left(\sum_{j=0}^{q} v^{j} h^{j}\right)\right|_{h=0}=\phi^{\prime}\left(v^{0}\right) v^{k}+\tilde{\Psi}^{k}\left(v^{0}, \ldots, v^{k-1}\right) .
$$

The proof is easily obtained, by writing

$$
g(h)=\sum_{i=0}^{q} v^{i} h^{i}
$$

and computing $d^{k} \phi(g(h)) / d h^{k}$. We shall use more often the notation $\Psi^{k}$ for a function slightly different of $\tilde{\Psi}^{k}$. We postpone its definition until needed (see (3.27).

3.2. Geometrical preliminaries. Let $\Gamma$ be a compact smooth manifold of dimension $N-1$ embedded in $\mathbb{R}^{N}$. $\Gamma$ is not necessarily connected. The manifold $\Gamma$ is equipped with the metric induced by the metric of $\mathbb{R}^{N}$. There is an intrinsic operator $\Delta^{\Gamma}$ defined on $C^{2}(\Gamma)$ as follows: let $\left(X_{\tau}, \tau\right)$ be a local chart on $\Gamma$, with $\tau\left(X_{\tau}\right)$ an open subset of $\mathbb{R}^{N-1}$. In local coordinates, the contravariant metric tensor is denoted $g_{\tau}^{j k}$ and $g_{\tau}=\operatorname{det}\left(g^{j k}\right)$; then

$$
\Delta^{\Gamma} v=\frac{1}{\sqrt{g_{\tau}}} \sum_{j} \frac{\partial}{\partial x^{j}}\left(\sqrt{g_{\tau}} g_{\tau}^{j k} \frac{\partial}{\partial x^{k}}\left(v \circ \tau^{-1}\right)\right) \circ \tau .
$$

It is well known that $\Delta^{\Gamma}$ depends smoothly on $\Gamma$ and the metric of $\Gamma$.

Let $\nu(\sigma)$ be a continuous normal at $\sigma$ to $\Gamma$, and define a mapping $Y$ from $\mathbb{R} \times \Gamma$ to $\mathbb{R}^{N}$ by $Y(\lambda, \sigma)=\sigma+\lambda \nu(\sigma)$. Let $\bar{\lambda}$ be strictly smaller than the infimum of the radii of curvature of $\Gamma$. Then, $Y$ is a diffeomorphism from $[-\bar{\lambda}, \bar{\lambda}] \times \Gamma$ 


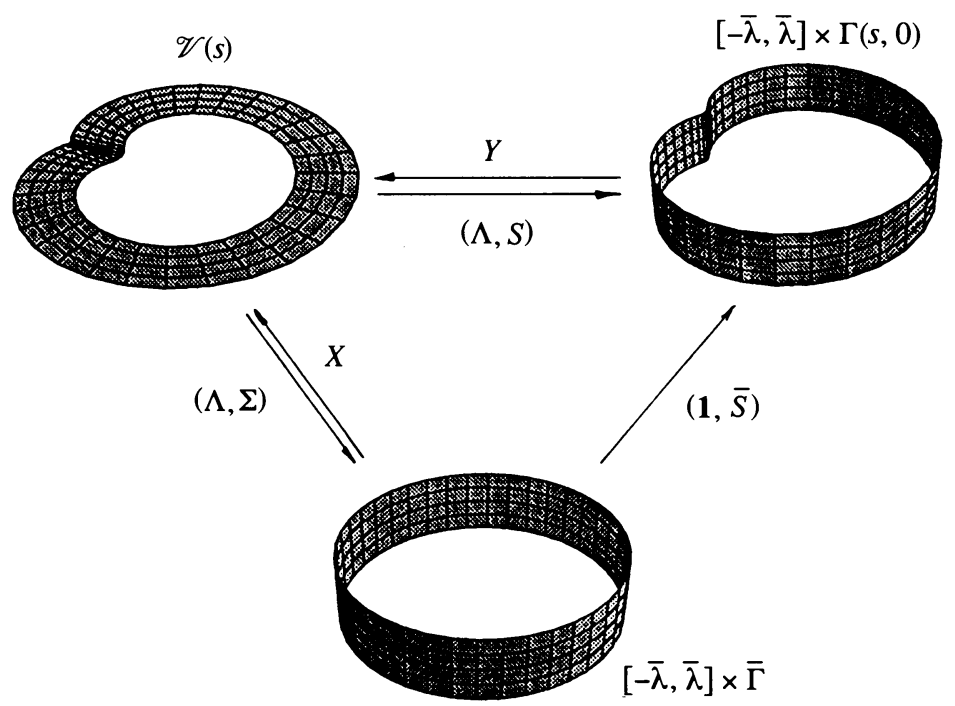

FIGURE 3.1.Tubular neighbourhoods, cylinders and all that

to a tubular neighborhood $\mathscr{V}$ of $\Gamma$; its inverse is denoted $(\Lambda(x), S(x))$. It is clear that $|\nabla \Lambda|=1$ on $\mathscr{V}$. We choose the direction of $\nu$ on each connected component of $\Gamma$ in such a way that $\Lambda$ can be extended as a smooth function on $\mathbb{R}^{N}$ which vanishes only on $\Gamma$.

Define for any $\lambda$ the manifold parallel to $\Gamma$

$$
\Gamma(\lambda)=\{Y(\lambda, \sigma) / \sigma \in \Gamma\} .
$$

If $|\lambda| \leq \bar{\lambda}, \Gamma(\lambda)$ is smooth. Then, the $N$-dimensional Laplace operator on $\mathscr{V}$ can be decomposed as $\Delta^{\perp}+\Delta^{\Gamma(\lambda)}$. More precisely, let $u$ be a smooth function on $\mathscr{V}$, and let

$$
v(\lambda, \sigma)=u(Y(\lambda, \sigma))
$$

Then

$$
(\Delta u)(Y(\lambda, \sigma))=\frac{\partial^{2} v}{\partial \lambda^{2}}+\frac{\partial v}{\partial \lambda} \Delta \Lambda(Y(\lambda, \sigma))+\left(\Delta^{\Gamma(\lambda)} v(\lambda, .)\right)(\sigma) .
$$

The above considerations carry over to the case in which the surface $\Gamma$ depends on parameters: in our case, since we expect that the (unknown) interface depends only on the slow time $s=h^{2} t$ and $h$, we shall denote it by $\Gamma(s ; h)$; it will be smooth, and we expect that there exist constants $T^{*}$ and $h^{*}$ to be determined later such that $\Gamma(s ; h)$ will stay diffeomorphic to itself for $s \leq T^{*}$ and for $h \leq h^{*}$. Hence, and in all the article, we will mention no more those limits, and the reader will understand that they hold whenever we write " for all $s$ and $h$ ". Let $\bar{\lambda}$ be strictly smaller than the infimum of the curvature radii of $\Gamma(s ; h)$, for all $h$. Let $\mathscr{V}^{0}(s)=\left\{x \in \mathbb{R}^{N} / \operatorname{dist}(x, \Gamma(s ; 0)) \leq \bar{\lambda}\right\}$. By possibly redefining $\bar{\lambda}$, we see that

$$
\left\{x \in \mathbb{R}^{N} / \operatorname{dist}(x, \Gamma(s ; h)) \leq \bar{\lambda}\right\} \subset \mathscr{V}^{0}(s), \quad \text { for all } h .
$$

In $\mathscr{V}^{0}(s)$, the normal (respectively tangential) coordinate $\Lambda(x, s ; h)$ (resp. $S(x, s ; h))$ is smooth; the diffeomorphism $Y(\lambda, \sigma, s ; h)$ and the parallel hypersurface $\Gamma(\lambda, s ; h)$ are defined as in the previous subsection. 
Let $\bar{\Gamma}$ be a reference manifold, assuming it is diffeomorphic to $\Gamma(s ; h)$ for all $s$ and all $h$. There is a diffeomorphism $\bar{S}(., s ; h)$ from $\bar{\Gamma}$ to $\Gamma(s ; h)$. We shall denote

$$
\begin{aligned}
X(\lambda, \sigma, s ; h) & =Y(\lambda, \bar{S}(\sigma, s ; h), s ; h), \\
\Sigma(x, s ; h) & =\bar{S}(., s ; h)^{-1} S(x, s ; h) .
\end{aligned}
$$

With these notations,

$$
\begin{gathered}
X(\Lambda(x, s ; h), \Sigma(x, s ; h), s ; h)=x, \quad \forall x \in \mathscr{V}^{0}(s), \forall s, \forall h ; \\
|\nabla \Lambda(x, s ; h)|=1, \quad \forall x \in \mathscr{V}^{0}(s), \forall s, \forall h .
\end{gathered}
$$

It turns out that a purely geometrical normalization conditions determines the parameterization $X(\lambda, \sigma, s ; h)$ of $\Gamma(s ; h)$ in terms of the normal coordinate $\Lambda(x, s ; h):$ more precisely, we have

Lemma 3.3. Assume $\Lambda(x, s ; h)$ is given, $\bar{S}(\sigma, 0 ; h)$ is independent of $h$, and the parametrization $X$ satisfies

$$
\frac{\partial X}{\partial s}(0, \sigma, s ; h) \text { is parallel to } \nabla \Lambda(X(0, \sigma, s ; h) s ; h), \quad \forall x \in \mathscr{V}^{0}(s), \forall s .
$$

Then the parametrization of $\Gamma(s ; h)$ is defined up to a reparametrization of $\bar{\Gamma}$ which is independent of $s$ and $h$.

Proof. Let $m(\sigma, s ; h)$ be a reparametrization of $\bar{\Gamma}$, i.e., a diffeomorphism of $\bar{\Gamma}$ to itself depending smoothly on $s$ and $h$, and let

$$
\tilde{X}(\lambda, \sigma, s ; h)=X(\lambda, m(\sigma, s ; h), s ; h) .
$$

Then (3.10) implies that

$$
\frac{\partial X}{\partial s}(0, m(\sigma, s ; h), s ; h) \text { is parallel to } \nabla \Lambda(\tilde{X}(0, \sigma, s ; h), s ; h) .
$$

On the other hand,

$$
\begin{aligned}
\frac{\partial \tilde{X}}{\partial s}(0, \sigma, s ; h)= & \frac{\partial m}{\partial s}(\sigma, s ; h) \cdot X(0, m(\sigma, s ; h), s ; h) \\
& +\frac{\partial X}{\partial s}(0, m(\sigma, s ; h), s ; h)
\end{aligned} .
$$

Therefore, the tangential vector $\partial m / \partial s(\sigma, s ; h) \cdot X(0, m(\sigma, s ; h), s ; h)$ has to be parallel to $\nabla \Lambda(\tilde{X}(0, \sigma, s ; h), s ; h)$ which is a normal vector. This implies $\partial m(\sigma, s ; h) / \partial s=0$, and $m(\sigma, s ; h)=m(\sigma, 0 ; h)$. In other words, $m$ is fixed by its initial condition. But we assumed $m(\sigma, 0 ; h)=m(\sigma, 0 ; 0)$. Thus $X(\lambda, \sigma, s ; h)$ is determined up to a constant reparametrization of $\bar{\Gamma}$, and so is $\Sigma(x, s ; h)$. This proves the claim.

Let $w$ be a smooth function of $(\mu, \sigma, t, s ; h) \in[-\bar{\lambda} / h, \bar{\lambda} / h] \times \Gamma(s ; h) \times$ $\mathbb{R}^{+} \times\left[0, T^{*}\right] \times\left[0, h^{*}\right]$, and let

$$
u(x, t ; h)=w\left(\frac{\Lambda\left(x, h^{2} t ; h\right)}{h}, S\left(x, h^{2} t ; h\right), t, h^{2} t ; h\right) .
$$


We have a formula analogous to (3.7), which involves $h$ :

$$
\begin{aligned}
h^{2} \Delta u(Y(\lambda, \sigma, s ; h), t ; h)= & \frac{\partial^{2} w}{\partial \mu^{2}}(\mu, \sigma, t, s ; h)|\nabla \Lambda(Y(h \mu, \sigma, s ; h), s ; h)|^{2} \\
& +h \frac{\partial w}{\partial \mu}(\mu, \sigma, t, s ; h) \Delta \Lambda(Y(\mu h, \sigma, s ; h), s ; h) \\
& +h^{2}\left(\Delta^{\Gamma(\mu h, s ; h)} w(\mu, ., t, s ; h)\right)(\sigma) .
\end{aligned}
$$

Let $v$ be the pull-back of $w$ by $X(\mu h, ., s ; h)$ :

$$
v(\mu, \sigma, t, s ; h)=w(\mu, X(\mu h, \sigma, s ; h), t, s ; h)=\left(X(\mu h, ., s ; h)_{*} w\right)(\sigma) .
$$

We define an elliptic differential operator $B(\mu, s ; h)$ on the fixed manifold $\bar{\Gamma}$ by pulling back $\Delta^{\Gamma(\mu h, s ; h)}$ to $\bar{\Gamma}$ using $X(\mu h, ., s ; h)$ :

$$
\begin{aligned}
B(\mu, & ; h) v(\mu, ., t, s ; h) \\
& =\Delta^{\Gamma(\mu h, s ; h)} w(\mu, ., t, s ; h) \\
& =X(\mu h, ., t, s ; h)_{*} \Delta^{\Gamma(\mu h, s ; h)} X(\mu h, ., s ; h)_{*}^{-1} v(\mu, ., t, s: h) .
\end{aligned}
$$

It appears from this formula that $B$ depends smoothly on $s, \mu$ and $h$, and is uniformly elliptic as long as $\Gamma(s ; h)$ is diffeomorphic to $\bar{\Gamma}$. It is convenient, at this point to introduce the notations

$$
\begin{aligned}
& L(\mu, \sigma, s ; h)=\left(\frac{\partial \Lambda}{\partial s}-\Delta \Lambda\right)(X(\mu h, \sigma, s ; h), s ; h) \\
& T(\mu, \sigma, s ; h)=\frac{\partial \Sigma}{\partial s}(X(h \mu, \sigma, s ; h), s ; h)
\end{aligned}
$$

Here, $\partial \Sigma / \partial s$ has an obvious meaning as an element of the tangent bundle of $\bar{\Gamma}$.

We look for a solution $u$ of (2.2) with initial condition (3.1) of the form

$$
u(x, t ; h)=v\left(\frac{\Lambda\left(x, h^{2} t ; h\right)}{h}, \Sigma\left(x, h^{2} t ; h\right), t, h^{2} t ; h\right) .
$$

Such a solution must satisfy the equation

$$
\frac{\partial v}{\partial t}-\frac{\partial^{2} v}{\partial \mu^{2}}+h L \frac{\partial v}{\partial \mu}+h^{2}\left(T \cdot v-B v+\frac{\partial v}{\partial s}\right)+\phi(v)=0
$$

As usual, the notation $T \cdot v$ denotes the action of the vector field $T$ on $v$. 3.3. Setting up the asymptotic expansion. The functions $v, \Lambda$ and $\Sigma$ are expanded in powers of $h$ as:

$$
\begin{gathered}
v(\mu, \sigma, t, s ; h) \sim \sum_{j \geq 0} v^{j}(\mu, \sigma, t, s) h^{j}, \\
\left\{\begin{array}{l}
\Lambda(x, s ; h) \sim \sum_{j \geq 0} \Lambda^{j}(x, s) h^{j}, \\
\Sigma(x, s ; h) \sim \sum_{j \geq 0} \Sigma^{j}(x, s) h^{j} .
\end{array}\right.
\end{gathered}
$$


Whenever necessary, $\Lambda^{j}$ (respectively, $\Sigma^{j}$ ) will be identified to a vector field, i.e., an operator of derivation in the $\lambda$ (respectively, $\sigma$ ) variable. In such case, we shall write $\Lambda^{j}$. (respectively, $\Sigma^{j} \cdot$ ).

We shall make use of the following auxiliary expansions

$$
\left\{\begin{aligned}
X(\lambda, \sigma, s ; h) & \sim \sum_{j \geq 0} X^{j}(\lambda, \sigma, s) h^{j}, \\
L(\mu, \sigma, s ; h) & \sim \sum_{j \geq 0} L^{j}(\mu, \sigma, s) h^{j}, \\
T(\mu, \sigma, s ; h) & \sim \sum_{j \geq 0} T^{j}(\mu, \sigma, s) h^{j}, \\
B(\mu, s ; h) & \sim \sum_{j \geq 0} B^{j}(\mu, s) h^{j} .
\end{aligned}\right.
$$

All the operators $B^{j}(s)$ are second order partial differential operators over $\bar{\Gamma}$ with smooth coefficients. In particular, they are all dominated by $\bar{B}=B^{0}(0)=$ $\Delta^{\Gamma}$.

If convenient we shall write

$$
\begin{aligned}
& v \text { or } v(h) \text { in place of } v(., ., ., . ; h), \\
& \Lambda \text { or } \Lambda(h) \text { in place of } \Lambda(., . ; h), \text { etc. }
\end{aligned}
$$

To write the equations for $v^{j}, \Lambda^{j}$, etc., we shall treat the slow time variable $s=h^{2} t$ and the fast time variable $t$ as independent quantities. This formal procedure, which is a standard feature of multiscale expansions, will be justified by the results of $\S 4$.

Using the expansions for $v(h), L(h), S(h), T(h)$ and formula (3.19), we obtain the equation satisfied by the $k$ th order term of the development of $v(h)$ as follows. The operator $A$ on $L^{2}(\mathbb{R})$ has been defined at (1.6); we denote by $\Pi$ the orthogonal projection on $\operatorname{ker} A=\mathbb{R} \theta$.

Initially, $\Gamma(0 ; h)=\Gamma_{0}$ for all $h$; therefore, $\Lambda(x, 0 ; h)=\Lambda_{0}(x)$. It is natural to take $\bar{S}(\sigma, 0 ; h)$ independent of $h$, and we will make this assumption.

For $k=0$ the equation is

$$
\frac{\partial}{\partial t} v^{0}-\frac{\partial^{2} v^{0}}{\partial \mu^{2}}+\phi\left(v^{0}\right)=0
$$

with initial condition

$$
v^{0}(\mu, \sigma, 0, s)=\Theta(\mu)
$$

while for $k \geq 1$ the equation is

$$
\begin{aligned}
\left(\frac{\partial}{\partial t}+A\right) v^{k}=- & \left\{\frac{\partial v^{k-2}}{\partial s}+\sum_{j=0}^{k-1} \frac{\partial v^{k-j-1}}{\partial \mu} L^{j}\right. \\
& \left.\quad+\sum_{j=0}^{k-2}\left(T^{j} \cdot v^{k-j-2}+B^{j} v^{k-j-2}\right)+\tilde{\Psi}^{k}\left(v^{0}, \ldots, v^{k-1}\right)\right\}
\end{aligned}
$$

with initial condition

$$
v^{k}(\mu, \sigma, 0, s)=0
$$


Let us consider first the equation for $v^{0}$. With the initial condition (3.21), $\boldsymbol{\Theta}(\mu)$ is a solution, so that, by uniqueness:

$$
v^{0}(\mu, \sigma, t, s)=\Theta(\mu) .
$$

The next equation reads

$$
\left(\frac{\partial}{\partial t}+A\right) v^{1}=-\frac{\partial v^{0}}{\partial \mu} L^{0}
$$

where

$$
L^{0}(\mu, \sigma, s)=\left(\frac{\partial \Lambda^{0}}{\partial s}-\Delta \Lambda^{0}\right)\left(X^{0}(0, \sigma, s), s\right) .
$$

To obtain a bounded solution for all $t \geq 0$, we require

$$
(\mathbb{I}-\Pi) P^{1}=0
$$

that is

$$
\int\left(-\frac{\partial v^{0}}{\partial \mu} L^{0}\right) L^{0} d \mu=0
$$

or, since $L^{0}$ is independent of $\mu$ :

$$
\begin{gathered}
\left(\frac{\partial}{\partial s}-\Delta\right) \Lambda^{0}\left(X^{0}(0, \sigma, s), s\right)=0, \\
\Lambda^{0}(x, 0)=\Lambda_{0}(x) \quad \text { for } x \in \Gamma_{0} .
\end{gathered}
$$

Observe that this partial differential equation holds only over some unknown submanifold of $\mathbb{R}^{N}$, denoted henceforth as

$$
\Gamma^{0}(s)=\left\{x \in \mathbb{R}^{N} / \Lambda^{0}(x)=0\right\}=\left\{X^{0}(0, \sigma, s) / \sigma \in \bar{\Gamma}\right\} .
$$

The initial condition is $\Gamma^{0}(0)=\Gamma_{0}$. Equation (3.25) is certainly insufficient to solve for $\Lambda^{0}$. We couple (3.25) with the constraint

$$
\left|\nabla \Lambda^{0}(x, s)\right|=1, \quad \forall x \in \mathscr{V}^{0}(s), \forall s,
$$

which is but the lowest order term of (3.9).

We have seen in the introduction that (3.24)-(3.26) describes motion by mean curvature of the hypersurface $\Gamma^{0}(s)$, parameterized by $\sigma \mapsto X^{0}(0, \sigma, s)$. According to the discussion of subsection 1.2, the motion by mean curvature has a smooth solution locally in time, i.e., for $s<T_{\text {sing }}$. From now on, we shall fix $T^{*}$ to be any positive number strictly less than $T_{\text {sing }}$.

By Lemma 3.3 we know that the parametrization of $\Gamma^{0}(s)=\Gamma(s ; 0)$ by $\bar{\Gamma}$ is fixed. As a consequence of the same lemma is that we can determine $\Sigma^{j}$ and $X^{j}$ in terms of $\Lambda^{k}$ (for $k \leq j$ ) and $X^{k}, \Sigma^{k}$ (for $\left.k \leq j-1\right)$ :

Lemma 3.4. There is a matrix $G$ with smooth coefficients depending only on $X^{0}$ and its derivatives such that

$$
\frac{\partial X^{j}}{\partial s}=G X^{j}+\tilde{Q}^{j}
$$

where $\tilde{Q}^{j}$ is polynomial in $\Lambda^{n}$ for $n \leq j$ and its derivatives and in $X^{n}, \Sigma^{n}$ for $n \leq j-1$ and their derivatives.

Proof. Let $\sigma_{1}, \ldots \sigma_{N-1}$ be local coordinates on $\bar{\Gamma}$. The parallelism relation (3.10) can be translated into

$$
\frac{\partial X}{\partial s}(\lambda, \sigma, s ; h) \frac{\partial X}{\partial \sigma^{k}}(\lambda, \sigma, s ; h)=0, \quad \forall k \in\{1, \ldots N-1\} .
$$


We differentiate $j$ times this relation with respect to $h$ and we obtain

$$
\frac{\partial X^{j}}{\partial \sigma^{k}} \frac{\partial X^{0}}{\partial s}+\frac{\partial X^{0}}{\partial \sigma^{k}} \frac{\partial X^{j}}{\partial s}+\bar{Q}_{k}^{j}=0
$$

where $\bar{Q}_{k}^{j}$ is polynomial in the $X^{n}$ 's and their derivatives for $n \leq j-1$.

On the other hand, differentiating $j$ times with respect to $h$ gives

$$
X^{j}+\sum_{k} \frac{\partial X^{0}}{\partial \sigma^{k}} \Sigma_{k}^{j}+Q^{j}=0
$$

where $Q^{j}$ is polynomial in $\Lambda^{n}$ for $n \leq j$ and its space derivatives, and in $\Sigma^{n}$ and $X^{n}$ for $n \leq j-1$ and their derivatives.

We differentiate (3.29) with respect to $\sigma^{m}$ and obtain

$$
\frac{\partial X^{j}}{\partial \sigma^{m}}+\sum_{k} \frac{\partial^{2} X^{0}}{\partial \sigma^{k} \partial \sigma^{m}} \Sigma_{k}^{j}+\sum_{k} \frac{\partial X^{0}}{\partial \sigma^{k}} \frac{\partial \Sigma_{k}^{j}}{\partial \sigma^{m}}+\frac{\partial Q_{k}^{j}}{\partial \sigma^{m}}=0
$$

We multiply this relation scalarly by $\left(\partial X^{0} / \partial s\right)$ and with the help of (3.29) we get the relation

$$
\frac{\partial X^{j}}{\partial s} \frac{\partial X^{0}}{\partial \sigma^{m}}=\sum_{k} \frac{\partial^{2} X^{0}}{\partial \sigma^{k} \partial \sigma^{m}} \Sigma_{k}^{j}+\frac{\partial Q_{k}^{j}}{\partial \sigma^{m}} \frac{\partial X^{0}}{\partial s}-\bar{Q}_{m}^{j}
$$

because $\left(\partial X^{0} / \partial \sigma^{k}\right)\left(\partial X^{0} / \partial s\right)$ vanishes.

On the other hand, we differentiate (3.29) with respect to $s$ and multiply scalarly by $\left(\partial X^{0} / \partial \lambda\right)$; since $\left(\partial X^{0} / \partial \sigma^{k}\right)\left(\partial X^{0} / \partial \lambda\right)$ vanishes, this yields

$$
\frac{\partial X^{j}}{\partial s} \frac{\partial X^{0}}{\partial \lambda}+\sum_{k} \frac{\partial^{2} X^{0}}{\partial \sigma^{k} \partial s} \Sigma_{k}^{j}+\frac{\partial Q_{k}^{j}}{\partial s} \frac{\partial X^{0}}{\partial \lambda}=0 .
$$

Relations (3.29) for $k=1$ to $N-1$ are a system of $N$ equations in the $N-1$ unknowns $\Sigma_{k}^{k}$. This system is of rank $N-1$ because the $N-1$ vectors $\left(\partial X^{0} / \partial \sigma^{k}\right)_{1 \leq k \leq N-1}$ are linearly independent. Therefore

$$
\left(\Sigma_{k}^{j}\right)_{1 \leq k \leq N-1}=F\left(Q^{j}+X^{j}\right)
$$

where $F$ is a matrix with smooth coefficients depending only on $X^{0}$. Together with (3.32), (3.30), (3.31) can be written in the form (3.27), where $\tilde{Q}^{j}$ depends only on the $X^{n}$ 's and their space derivatives for $n \leq j-1$, the $\lambda^{n}$ 's and their space derivatives for $n \leq j$, and $G$ is a matrix with smooth coefficients depending only on $X^{0}$ and its first derivatives.

Corollary 3.5. If $X^{n}, \Sigma^{n}(n \leq j-1)$ and $\Lambda^{n}(n \leq j)$ are smooth, then $X^{j}$ and $\Sigma^{j}$ are smooth.

Proof. Recall that $X^{j}(0, \sigma, 0)=0$. Then we integrate (3.27), and it is clear from (3.32) that $\Sigma^{j}$ is smooth.

Once (3.24) $-(3.26)$ is solved, the equation for $v_{1}$ reads

$$
\left(\frac{\partial}{\partial t}+A\right) v^{1}=0
$$


which together with the initial condition $v^{1}(\mu, \sigma, 0, s)=0$ implies $v^{1} \equiv 0$. As a consequence, $\tilde{\Psi}^{k}$ depends only on $v^{0}, \ldots, v^{k-2}$ for $k \geq 2$, since the only $\alpha \in A_{k}$ having a $(k-1)$ th nonzero entry is $(1,0, \ldots, 0,1,0)$. From now on, we define

$$
\Psi^{k}\left(v^{0}, v^{2}, \ldots, v^{k-2}\right)=\tilde{\Psi}^{k}\left(v^{0}, 0, v^{2}, \ldots, v^{k-2}, v^{k-1}\right) .
$$

To solve the $k$ th equation for $k \geq 1$ the procedure is similar: define $-P^{k}$ as the right-hand side of (3.22), namely

$$
\begin{aligned}
P^{k}= & \sum_{j=0}^{k-1} \frac{\partial v^{k-j-1}}{\partial \mu} L^{j}+\sum_{j=0}^{k-2}\left(T^{j} \cdot v^{k-j-2}+B^{j} v^{k-j-2}\right) \\
& +\Psi^{k}\left(v^{0}, \ldots, v^{k-2}\right)+\frac{\partial v^{k-2}}{\partial s} .
\end{aligned}
$$

As $L^{0}$ vanishes, $P^{k}$ does not involve $L^{k}$. Therefore $P^{k}$ depends on $v^{j}$, $\partial v^{j} / \partial \mu, B^{l}, T^{j}$ only for $j, l \leq k-2$, and $L^{j}$ for $j \leq k-1$.

3.4. The recursion algorithm: equations for the $\Lambda^{j}$ 's. We need to understand the $L^{l}$ 's in terms of the $\Lambda^{j}$ 's and other slow quantities. The relevant information is contained in Lemma 3.6. We need a definition: the trace of the third fundamental form of $\Gamma^{0}(s)$, or equivalently of the square of the Weingarten map, is denoted $C(\sigma, s)$; it is given by

$$
C(\sigma, s)=\sum_{j=1}^{N-1} \kappa_{j}^{2}(\sigma, s),
$$

where $\left(\kappa_{j}\right)_{1 \leq j \leq N-1}$ denotes the scalar curvatures at the point $\sigma$ and at the time $s$.

Lemma 3.6. For any $l \geq 1$,

$$
L^{l}(\mu, \sigma, s)=\left(\frac{\partial}{\partial s}-\Delta-C(\sigma, s)\right) \Lambda^{l}(x, s)+Q_{0}^{l}(\mu, \sigma, s),
$$

where $Q_{0}^{l}$ is polynomial with respect to $\mu$ and with respect to $\Lambda^{j}, \Sigma^{j}, X^{j}$ (and their derivatives in $x$ and $s, x, \lambda$ respectively) for $j \leq l-1$, and $x$ is evaluated on $\Gamma^{0}(s)$.

Proof. Let us define

We have

$$
\begin{aligned}
M(x, s ; h) & =\left[\left(\frac{\partial}{\partial s}-\Delta\right) \Lambda\right](x, s ; h), \\
M^{j}(x, s) & =\left[\left(\frac{\partial}{\partial s}-\Delta\right) \Lambda^{j}\right](x, s) .
\end{aligned}
$$

$$
L(\mu, \sigma, s ; h)=M(X(h \mu, \sigma, s ; h), s ; h) .
$$

To calculate the successive derivatives of $L$ with respect to $h$, we shall apply Lemma 3.2:

$$
\begin{aligned}
L^{l} l ! & =\left.\frac{\partial^{l}}{\partial h^{l}}(\mu, \sigma, s ; h)\right|_{h=0}=l ! M^{l}\left(X^{0}(0, \sigma, s), s\right) \\
& +\sum_{p=1}^{l} C_{l}^{p} \sum_{\alpha \in A_{p}} a_{\alpha}^{p}(l-p) ! D_{x}^{|\alpha|} M^{l-p} \bigotimes_{j=1}^{p}\left(\left.\frac{\partial^{j} X}{\partial h^{j}}(h \mu, \sigma ; h)\right|_{h=0}\right)^{\otimes \alpha_{j}} .
\end{aligned}
$$


Let us define $Q_{1}^{l}$ by

$$
L^{l}(\mu, \sigma, s)=M^{l}\left(X^{0}(0, \sigma, s), s\right)+Q_{1}^{l} .
$$

To obtain a more explicit expression of $Q_{1}^{l}$ we calculate

$$
\left.\frac{\partial^{j} X}{\partial h^{j}}(h \mu, \sigma ; h)\right|_{h=0}=\sum_{s=0}^{j} C_{j}^{s} s ! \frac{\partial^{j-s} X^{s}}{\partial \lambda^{j-s}} \mu^{j-s} .
$$

Careful attention to the indices shows that

$$
Q_{1}^{l}=\nabla M^{0} X^{l}+Q_{2}^{l},
$$

where $Q_{2}^{l}$ depends polynomially on $X^{q}, M^{q}$ (and their respective derivatives in $x$ and $\lambda$ ) only for $q \leq l-1$. Then

$$
X^{l}=-\frac{\partial X^{0}}{\partial \lambda} \Lambda^{l}+Q_{3}^{l},
$$

where $Q_{3}^{l}$ is a sum of differential monomials of the form

$$
\Lambda^{p_{1}} \otimes \cdots \otimes \Lambda^{p_{r}} \otimes \Sigma^{q_{1}} \otimes \cdots \otimes \Sigma^{q_{m}} \cdot X^{k}
$$

where $p_{1}+\cdots+p_{r}+q_{1}+\cdots+q_{m}+k \leq l$, and $k \leq l-1$. Here the symbols $\Lambda^{p_{i}}$ and $\Sigma^{q_{j}}$ denote differential operators respectively in $\lambda$ and $\sigma$; the tensor product of the $\Lambda^{p_{i}}$ 's has a clear meaning because it differentiates in one dimension; the tensor product of the $\Sigma^{q_{j}}$ 's understates the covariant structure of $\Gamma^{0}(s)$ defined by the obvious Riemannian connection on $\Gamma^{0}(s)$ induced by the metric of $\mathbb{R}^{N}$.

Relation (3.33) follows simply by differentiating (3.10) $l$ times with respect to $h$ and taking its value at $h=0$.

Denote by $\nu(\sigma, s)$ the exterior unit normal at time $s$ to $\Gamma^{0}(s)$; then by construction (see subsection 3.2)

$$
X^{0}(\lambda, \sigma, s)=X^{0}(0, \sigma, s)+\lambda \nu(\sigma, s)
$$

and

$$
\frac{\partial}{\partial \lambda} X^{0}(\lambda, \sigma, s)=\nu(\sigma, s)=\nabla \Lambda^{0}(0, \sigma, s) .
$$

From (3.31), (3.33), (3.34) we conclude

$$
L^{l}=M^{l}-\nabla \Lambda^{0} \nabla M^{0} \Lambda^{l}+Q_{0}^{l}
$$

where $Q_{0}^{l}$ has the required property; recalling the definition of $M^{l}$ :

$$
L^{l}=\left(\partial_{s}-\Delta\right) \Lambda^{l}-\left[\nabla \Lambda^{0} \nabla M^{0}\right] \Lambda^{l}+Q_{0}^{l} .
$$

To compute $\nabla \Lambda^{0} \nabla M^{0}=\nabla \Lambda^{0} \nabla(\partial / \partial s-\Delta) \Lambda^{0}$, observe that

$$
\nabla \Lambda^{0} \nabla \frac{\partial}{\partial s} \Lambda^{0}=\frac{1}{2} \frac{\partial}{\partial s}\left|\nabla \Lambda^{0}\right|^{2}=0 ;
$$

as to $-\nabla \Lambda^{0} \nabla \Delta \Lambda^{0}$, this term can be written as

$$
-\sum_{j} \frac{\partial}{\partial x_{j}}\left(\frac{\partial}{\partial x_{j}} \nabla \Lambda^{0} \nabla \Lambda^{0}\right)+\sum_{j}\left|\frac{\partial \nabla \Lambda^{0}}{\partial x_{j}}\right|^{2}=\sum_{j}\left|\frac{\partial \nabla \Lambda^{0}}{\partial x_{j}}\right|^{2} .
$$


The last expression is equal to the sum $C(\sigma, s)$ of the squares of the principal curvatures of $\Gamma^{0}(s)$, as a direct calculation in local coordinates can show.

A consequence of the above result is that we can write

$$
P^{k}=\theta\left(\frac{\partial}{\partial s}-\Delta-C(\sigma, s)\right) \Lambda^{k-1}+P_{1}^{k},
$$

where $P_{1}^{k}$ depends only on quantities $v^{j}, \Lambda^{j}$, etc. of order $j \leq k-2$. An orthogonality condition similar to (3.23) will lead to a partial differential equation on $\Gamma^{0}(s)$, as will be shown in subsection 3.4 below; as at order 0 , this is not enough to solve for $\Lambda^{l}$. Therefore, one needs the analogue of (3.25): this is simply the term of order $l$ of (3.11) squared, i.e.

$$
\sum_{p=0}^{l} \nabla \Lambda^{l-p} \nabla \Lambda^{p}=0 \quad \text { on } \mathscr{V}^{0}(s) .
$$

The next lemma ensures the unique solvability of equations of the form

$$
\begin{array}{r}
\left(\frac{\partial}{\partial s}-\Delta-C(\sigma, s)\right) \Lambda^{l}(x, s)=R^{l}(x, s), \\
\forall x \in \Gamma^{0}(s), \forall s \in\left[0, T^{*}\right] ;
\end{array}
$$

Lemma 3.7. Problem (3.37), (3.38), (3.39) is equivalent to a linear uniformly parabolic problem on $\bar{\Gamma} \times\left[0, T^{*}\right]$, with smooth coefficients depending on $s$ and $\sigma$. Therefore, it admits a unique solution whenever $\tilde{\boldsymbol{R}}^{l}, R^{l}$ are sufficiently regular functions.

Proof. Let

$$
G^{l}(\lambda, \sigma, s)=\Lambda^{l}\left(X^{0}(\lambda, \sigma, s), s\right)-\Lambda^{l}\left(X^{0}(0, \sigma, s), s\right) .
$$

Then, we use (3.38) to see that

$$
\begin{aligned}
\frac{\partial G^{l}}{\partial \lambda}(\lambda, \sigma, s) & =\nabla \Lambda^{l}\left(X^{0}(\lambda, \sigma, s), s\right) \frac{\partial X^{0}}{\partial \lambda}(0, \sigma, s) \\
& =\nabla \Lambda^{l}\left(X^{0}(\lambda, \sigma, s), s\right) \nabla \Lambda^{0}\left(X^{0}(\lambda, \sigma, s), s\right) \\
& =\tilde{R}^{l}\left(X^{0}(\lambda, \sigma, s), s\right)
\end{aligned}
$$

and it is clear that $G^{l}$ is completely determined by

$$
G^{l}(\lambda, \sigma, s)=\int_{0}^{\lambda} \tilde{R}^{l}\left(X^{0}\left(\lambda^{\prime}, \sigma, s\right), s\right) d \lambda^{\prime} .
$$

Let

$$
F(\sigma, s)=\Lambda^{l}(\lambda, \sigma, s)-G^{l}(\lambda, \sigma, s) .
$$

If we substitute in (3.37) the decomposition $\Lambda^{l}=F^{l}+G^{l}$, we obtain

$$
\left(\frac{\partial}{\partial s}-B(0, s ; 0)-C\right) F^{l}=R^{l}-\left(\frac{\partial}{\partial s}-\Delta-C\right) G^{l} \text {. }
$$


Here, of course, $B(0, s ; 0)$ is the pull-back of $\Delta^{\Gamma^{0}(s)}$ to $\bar{\Gamma}$ via $X^{0}(0, \ldots, s)$, which is clearly a uniformly elliptic operator for $s \leq T^{*}$.

With the initial condition (3.39), (3.40) possesses a unique solution. This can be proved by appealing to results on elliptic operators on manifolds (see for instance [Gi] or [Jo]) and the theorem of Kato and Tanabe on the solution of evolution equation with variable coefficients [KaTa], or alternatively the variational approach of Lions [Li].

3.5. The recursion algorithm: equations for the $v^{j}$ 's. As we have seen, $P^{k}$ contains an operator acting on $\Lambda^{k-1}$ plus other terms containing $v^{j}$ (for $j \leq$ $k-2)$. Therefore, $P^{j}$ depends on the fast time variable $t$. Requiring, as we did in case $k=1$ (see (3.23)), that $P^{k}$ be orthogonal to $\theta$ would lead to an equation for $\Lambda^{k-1}$ with source terms depending on the fast time variable. To circumvent this difficulty, we define the secular part of $v^{k}$ as

$$
\hat{v}^{k}(\mu, \sigma, s)=\lim _{t \rightarrow \infty} v^{k}(\mu, \sigma, t, s),
$$

provided that this limit exists. Let $\Pi$ be the orthogonal projection on $\theta \mathbb{R}$ in $L^{2}(\mathbb{R})$, and let

$$
\begin{aligned}
\hat{P}^{k}= & \frac{\partial \hat{v}^{k-2}}{\partial s}+\sum_{j=0}^{k-1} \frac{\partial \hat{v}^{k-j-1}}{\partial \mu} L^{j}+\sum_{j=0}^{k-2}\left(T^{j} \cdot \hat{v}^{k-j-2}+B^{j} \hat{v}^{k-j-2}\right) \\
& +\Psi\left(\hat{v}^{0}, \ldots, \hat{v}^{k-2}\right) .
\end{aligned}
$$

We shall require that

$$
\Pi \hat{P}^{k}=0,
$$

and we shall show in $\S 4$ that this weaker requirement ensures that $v^{k}$ is bounded in suitable functional spaces, for all $t \geq 0$.

The only term involving $L^{k-1}$ in $\hat{P}^{k}$ is $\theta L^{k-1}$, because $v^{0}=\hat{v}^{0}=\theta$. We have seen at Lemma 3.7 that

$$
L^{k-1}=\left.\left(\frac{\partial}{\partial s}-\Delta-C(\sigma, s)\right) \Lambda^{k-1}\right|_{x \in \Gamma^{0}(s)}+Q^{k-1}
$$

Let

$$
\hat{P}_{1}^{k}=\hat{P}^{k}-\left.\theta\left(\frac{\partial}{\partial s}-\Delta-C(\sigma, s)\right) \Lambda^{k-1}\right|_{x \in \Gamma^{0}(s)} .
$$

Then (3.43) is equivalent to

$$
\left.\left(\frac{\partial}{\partial s}-\Delta-C(\sigma, s)\right) \Lambda^{k-1}\right|_{x \in \Gamma^{0}(s)}=-\frac{\left(\hat{P}_{1}^{k}, \theta\right)}{|\theta|_{2}^{2}},
$$

and simultaneously we impose

$$
\begin{gathered}
\nabla \Lambda^{k-1} \nabla \Lambda^{0}=-\frac{1}{2} \sum_{p=1}^{k-2} \nabla \Lambda^{k-p-1} \nabla \Lambda^{p} \text { on } \mathscr{V}^{0}(s), \\
\Lambda^{k-1}(., 0)=0 \text { on } \bar{\Gamma} .
\end{gathered}
$$


Finally, we define $v^{k}$ as the solution of

$$
\left\{\begin{array}{c}
\left(\partial_{t}+A\right) v^{k}=-P^{k} \\
v^{k}(\mu, \sigma, 0, s)=0
\end{array}\right.
$$

According to Lemma 3.7, the system (3.45), (3.46), (3.47) possesses a unique solution for $s \leq T^{*}$, provided that the right-hand side is known. But, by definition, $\hat{P}_{1}^{k}$ depends only on $\hat{v}^{j}, L^{j}, B^{j}$ and $T^{j}$ for $j \leq k-2$; therefore, the resolution of (3.45), (3.46), (3.47) and (3.48) for all $k$ will be possible inductively, at least at the formal level.

As mentioned in the Introduction, $v^{2}$ does not vanish in general; in fact, we can apply the results of this section to obtain

$$
\Lambda^{1}=0, \quad\left(\frac{\partial}{\partial t}+A\right) v^{2}=C(\sigma, s) \mu \theta(\mu) .
$$

Unless $\Gamma^{0}(s)$ is flat at some point, there is a second term in the expansion.

\section{ESTIMATES ON THE ASYMPTOTIC EXPANSION}

It is our purpose now to establish estimates in $L^{2}(\mathbb{R})$ and pointwise estimates on $v^{k}$ and its derivatives $\bar{B}^{\alpha_{1}} \partial^{\alpha_{2}} v^{k} / \partial s^{\alpha_{2}}$ and $\bar{B}^{\alpha_{1}} \partial^{\alpha_{2}+1} v^{k} / \partial s^{\alpha_{2}} \partial \mu$. The hilbertian estimates will enable us to see that the expansion is, indeed, asymptotic, namely that the $v^{k}$ and all their derivatives in the tangential variables $\sigma$ and $s$ are bounded in $L^{\infty}(\mathbb{R})$ uniformly with respect to $\mu, \sigma, t, s \in$ $\mathbb{R} \times \bar{\Gamma} \times \mathbb{R}^{+} \times\left[0, T^{*}\right]$ (subsection 4.1)

In subsection 4.2 , we consider first the following auxiliary problem: let $f(x, t)$ decrease exponentially to zero as $|x|$ tends to infinity, uniformly in time, and let $v$ be the solution of $\partial v / \partial t+A v=f$, where $A$ was defined at (1.7). If we assume that $|v(., t)|_{L^{2}(\mathbb{R})}$ is bounded independently of time, we show that $v(x, t)$ decreases exponentially to 0 as $|x|$ tends to infinity, uniformly in time.

Then we use this result to show that all the $v^{k}$ and their tangential derivatives decrease to 0 as $|\mu|$ tends to infinity, uniformly with respect to $(\sigma, t, s) \in$ $\bar{\Gamma} \times \mathbb{R}^{+} \times\left[0, T^{*}\right]$. Hence, we may extend the asymptotic expansion of $u$, which is defined only in a tubular neighborhood of $\Gamma^{0}(s)$ into a smooth function defined over all of $\mathbb{R}^{N}$ for $t \leq T^{*} h^{-2}$.

Finally, in subsection 4.3 , we estimate in $L^{2}\left(\mathbb{R}^{n}\right)$ and in $L^{\infty}\left(\mathbb{R}^{n}\right)$ the remainder

$$
\rho^{q, h}=\left(\frac{\partial}{\partial t}-\Delta\right) u^{q, h}+\phi\left(u^{q, h}\right),
$$

where $u^{q, h}$ is the asymptotic expansion with $q$ terms.

4.1. First estimates over $\Lambda^{k}, \Sigma^{k}, v^{k}$ and their derivatives in $\sigma$ and $s$. The construction algorithm for the $\Lambda^{k}, \Sigma^{k}$ and $v^{k}$ has been described in subsections 3.3 to 3.5. We shall need more information on the operator $A$ : let $\Pi$ be the orthogonal projection in $L^{2}(\mathbb{R})$ on $\mathbb{R} \theta$, and let the operator $A_{1}$ be the restriction of $A$ to $(\operatorname{ker} A)^{\perp}=\operatorname{ker} \Pi$. There is a strictly positive constant $\omega$ such that the operator norms of $A_{1}^{-1}$ and of $\exp \left(-t A_{1}\right)$ in $L^{2}(\mathbb{R})$ are bounded as follows:

$$
\left\|A_{1}^{-1}\right\| \leq \omega \quad \text { and } \quad\left\|\exp \left(-t A_{1}\right)\right\| \leq e^{-\omega t}, \quad \forall t \geq 0 .
$$


Moreover, there is a constant $C$ such that

$$
\left\|A_{1}^{1 / 2} \exp \left(-t A_{1}\right)\right\| \leq C \frac{e^{-\omega t}}{\sqrt{t}}, \quad t>0 .
$$

Theorem 4.1. Let $\Lambda^{k}, \Sigma^{k}$ and $v^{k}$ be constructed as in subsections 3.3 to 3.5. Then, the following conditions hold for all $j$ :

$$
\Lambda^{j} \text { is of class } C^{\infty} \text { over } \mathscr{V}^{0}(s) \times\left[0, T^{*}\right] \text {; }
$$$$
\Sigma^{j} \text { is of class } C^{\infty} \text { over } \mathscr{V}^{0}(s) \times\left[0, T^{*}\right] \text {; }
$$

$$
\left\{\begin{array}{l}
\forall \alpha=\left(\alpha_{1}, \alpha_{2}\right) \in \mathbb{N}^{2}, \text { there exist constants } \gamma_{j}<\omega \text { and } C_{j}(\alpha) \text { such that } \\
\left|\bar{B}^{\alpha_{1}} \frac{\partial^{\alpha_{2}} v^{j}}{\partial s^{\alpha_{2}}}(\mu, \sigma, t, s)\right|+\left|\bar{B}^{\alpha_{1}} \frac{\partial^{\alpha_{2}+1} v^{j}}{\partial s^{\alpha_{2}} \partial \mu}(\mu, \sigma, t, s)\right| \leq C_{j}(\alpha) \exp \left(\gamma_{j}|\mu|\right) \\
\forall(\mu, \sigma, t, s) \in \mathbb{R} \times \bar{\Gamma} \times \mathbb{R}^{+} \times\left[0, T^{*}\right] .
\end{array}\right.
$$

Proof. The proof is by induction. For $j=0,(4.5)_{0}$ and $(4.6)_{0}$ hold thanks to the results about motion by mean curvature; as $v^{0}=\theta$ and $v^{1}=0,(4.5)_{j}$ and $(4.6)_{j}$ are satisfied trivially for $j=0$ or 1 .

Assume that $(4.5)_{j}$ and $(4.6)_{j}$ hold for all $j \leq k-2$ and that $(4.5)_{j}$ and $(4.6)_{j}$ hold for all $j \leq k-1$. The induction hypothesis implies that the source terms in (3.45) and (3.46) are smooth. It follows from Lemma 3.7 that system (3.45), (3.46), and (3.47) has a smooth solution on $\mathscr{V}^{0}(s) \times\left[0, T^{*}\right]$. We can use now Corollary 3.5 to see that $\Sigma^{k-1}$ is smooth over $\mathscr{V}^{0}(s) \times\left[0, T^{*}\right]$.

Let us prove now that $(4.5)_{k}$ and $(4.6)_{k}$ hold. Define $\tilde{v}^{k}$ by

$$
\left(\partial_{t}+A\right) \tilde{v}^{k}=\hat{P}^{k}, \quad v^{k}(\mu, \sigma, 0, s)=0 .
$$

The orthogonality condition (3.43) implies that $\tilde{v}^{k}(t)$ is orthogonal to $\theta$ for all $t$; moreover, the source term in (4.7) does not depend on $t$, and (4.7) can be integrated readily as

$$
\tilde{v}^{k}(t)=-A_{1}^{-1}\left(\mathbb{I}-\exp \left(-t A_{1}\right)\right) \hat{P}^{k}
$$

Assumptions $(4.3)_{j},(4.4)_{j},(4.5)_{j}$ for $j \leq k-1$ imply that $\hat{P}^{k}(., \sigma, t, s)$ is bounded in $L^{2}(\mathbb{R})$, uniformly with respect to $\sigma, t$ and $s$. Therefore, it follows from (4.1) that $\tilde{v}^{k}$ is bounded in $L^{2}(\mathbb{R})$, uniformly with respect to $\sigma, t$ and $s$.

Let us prove now that $v^{k}$ is bounded in $L^{2}(\mathbb{R})$, uniformly with respect to $\sigma, t$ and $s$. We subtract (4.7) from (3.48) and obtain

$$
\left\{\begin{array}{l}
\left(\partial_{t}+A\right)\left(v^{k}-\tilde{v}^{k}\right)=\hat{P}^{k}-P^{k} \\
\left(v^{k}-\tilde{v}^{k}\right)(\mu, \sigma, 0, s)=0
\end{array}\right.
$$

whose solution is given by

$$
\left(v^{k}-\tilde{v}^{k}\right)(t)=\int_{0}^{t} \Pi\left(\hat{P}^{k}-P^{k}\right)\left(t^{\prime}\right) d t^{\prime}+\int_{0}^{t} \exp \left(-\left(t-t^{\prime}\right) A_{1}\right)\left(\hat{P}^{k}-P^{k}\right)\left(t^{\prime}\right) d t^{\prime}
$$


The induction hypotheses $(4.3)_{j},(4.4)_{j},(4.5)_{j}$ and $(4.6)_{j}$ for $j \leq k-1$ imply that $\hat{P}^{k}-P^{k}$ is bounded in $L^{2}(\mathbb{R})$, uniformly with respect to $\sigma, t$ and $s$. Thanks to (4.1), we can see that the second integral in (4.8) is bounded for all time $t$.

Therefore, we have only to estimate the first integral in (4.8), namely

$$
\int_{0}^{t} \Pi\left(\hat{P}^{k}-P^{k}\right)\left(t^{\prime}\right) d t^{\prime}
$$

Thanks to Sobolev estimates and the induction hypothesis, the $v^{j}$ 's are bounded for $j \leq k-1$ uniformly in $\mu, \sigma, t, s$. As $P^{k}$ depends polynomially over the $v^{j}$ 's and their derivatives for $j \leq k-2$, there exists a $\delta^{k}(0)>0$ such that

$$
\left|\hat{P}^{k}(., \sigma, s)-P^{k}(., \sigma, t, s)\right|_{2} \leq C \exp \left(-t \delta_{k}(0)\right),
$$

and we conclude immediately that (4.9) is bounded in $L^{2}(\mathbb{R})$, uniformly with respect to $\sigma, t$ and $s$.

Let us show now that $\partial v^{k} / \partial \mu$ is bounded in $L^{2}(\mathbb{R})$, uniformly with respect to $\sigma, t$ and $s$. We observe that for all $u$ in $H^{1}(\mathbb{R})$,

$$
\left|\frac{\partial v^{k}}{\partial \mu}\right|_{2} \leq C\left(\left|A^{1 / 2} u\right|_{2}+|u|_{2}\right)
$$

thus, it is enough to study $A^{1 / 2} v^{k}$ :

$$
A^{1 / 2} v^{k}(t)=-\int_{0}^{t} A_{1}^{1 / 2} \exp \left(-\left(t-t^{\prime}\right) A_{1}\right)(\mathbb{I}-\Pi) P^{k}\left(t^{\prime}\right) d t^{\prime} .
$$

Recalling property (4.2) of $A_{1}$, we can see that $A^{1 / 2} v^{k}(t)$ is bounded in $L^{2}(\mathbb{R})$, uniformly with respect to $\sigma, t$ and $s$. In particular, $v^{k}$ is bounded in $L^{\infty}\left(\mathbb{R} \times \bar{\Gamma} \times \mathbb{R}^{+} \times\left[0, T^{*}\right]\right)$.

To show that $v^{k}$ converges in $L^{2}(\mathbb{R})$ exponentially in time to a certain limit $\hat{v}^{k}$, as $t$ tends to infinity, we decompose $v^{k}(t)$ on $\mathbb{R} \theta$ and its orthogonal subspace:

$$
v^{k}(t)=-\int_{0}^{t} \exp \left(-A_{1}\left(t-t^{\prime}\right)\right)(\mathbb{I}-\Pi) P^{k}\left(t^{\prime}\right) d t^{\prime}+\int_{0}^{t} \Pi P^{k}\left(t^{\prime}\right) d t^{\prime} .
$$

The first integral in (4.11) is rewritten as follows:

$$
\begin{aligned}
\int_{0}^{t} \exp \left(-A_{1}\left(t-t^{\prime}\right)\right)(\mathbb{I}-\Pi) \hat{P}^{k}\left(t^{\prime}\right) d t^{\prime} \\
\quad+\int_{0}^{t} \exp \left(-A_{1}\left(t-t^{\prime}\right)\right)(\mathbb{I}-\Pi)\left(P^{k}\left(t^{\prime}\right)-\hat{P}^{k}\right) d t^{\prime} \\
=A_{1}^{-1}\left(\mathbb{I}-\exp \left(-A_{1} t\right)\right)(\mathbb{I}-\Pi) \hat{P}^{k} \\
\quad+\int_{0}^{t} \exp \left(-A_{1}\left(t-t^{\prime}\right)\right)(\mathbb{I}-\Pi)\left(P^{k}\left(t^{\prime}\right)-\hat{P}^{k}\right) d t^{\prime} \\
=A_{1}^{-1}(\mathbb{I}-\Pi) \hat{P}^{k}+\text { remainder },
\end{aligned}
$$

We deduce from the induction hypotheses that there exists a constant $\delta_{k}(0)$ such that

$$
\sup _{\sigma, s}\left|\hat{P}^{k}(., \sigma, t)-P^{k}(., \sigma, t, s)\right|_{2} \leq C \exp \left(-t \delta_{k}(0)\right) .
$$


This $\delta_{k}(0)$ is the minimum of a finite collection of $\gamma_{j}(\alpha)$, where $j$ runs between 0 and $k-1$, and the multiindices $\alpha$ are determined in terms of the dimension $N-1$ of $\Gamma$ through Sobolev estimates. Then the $L^{2}$ norm of the remainder is estimated by

$$
C\left(\exp (-\omega t)+\frac{\exp \left(-\delta_{k}(0) t\right)-\exp (-\omega t)}{\omega-\delta_{k}(0)}\right) \leq C \exp \left(-\delta_{k}(0) t\right) .
$$

The second integral in (4.11) can be rewritten as

$$
\int_{0}^{t} \Pi\left[P^{k}\left(t^{\prime}\right)-\hat{P}^{k}\right] d t^{\prime}
$$

which trivially converges as $t$ tends to infinity; moreover,

$$
\left|\int_{t}^{\infty} \Pi\left[P^{k}\left(t^{\prime}\right)-\hat{P}^{k}\right] d t^{\prime}\right| \leq \int_{t}^{\infty} C \exp \left(-\delta_{k}(0) t^{\prime}\right) d t^{\prime}=C \frac{\exp \left(-\delta_{k}(0) t\right)}{\delta_{k}(0)} .
$$

Therefore, $v^{k}$ converges exponentially in $t$ in $L^{2}(\mathbb{R})$, uniformly with respect to $\sigma$ and $s$, and its limit is

$$
\hat{v}^{k}=-A_{1}^{-1}(\mathbb{I}-\Pi) \hat{P}^{k}-\int_{0}^{\infty} \Pi P^{k}\left(t^{\prime}\right) d t^{\prime}
$$

We apply the same type of argument to $A^{1 / 2} v^{k}$ :

$$
\begin{aligned}
-A^{1 / 2} v^{k}(t)= & \int_{0}^{t} A_{1}^{1 / 2} \exp \left(-A_{1}\left(t-t^{\prime}\right)\right)(\mathbb{I}-\Pi) P^{k}\left(t^{\prime}\right) d t^{\prime} \\
= & \int_{0}^{t} A_{1}^{1 / 2} \exp \left(-A_{1}\left(t-t^{\prime}\right)\right)(\mathbb{I}-\Pi) \hat{P}^{k}\left(t^{\prime}\right) d t^{\prime} \\
& +\int_{0}^{t} A_{1}^{1 / 2} \exp \left(-A_{1}\left(t-t^{\prime}\right)\right)(\mathbb{I}-\Pi)\left(P^{k}\left(t^{\prime}\right)-\hat{P}^{k}\right) d t^{\prime} \\
= & A_{1}^{-1 / 2}\left(\mathbb{I}-\exp \left(-A_{1} t\right)\right)(\mathbb{I}-\Pi) \hat{P}^{k} \\
& +\int_{0}^{t} A_{1}^{1 / 2} \exp \left(-A_{1}\left(t-t^{\prime}\right)\right)(\mathbb{I}-\Pi)\left(P^{k}\left(t^{\prime}\right)-\hat{P}^{k}\right) d t^{\prime} \\
= & A_{1}^{-1 / 2}(\mathbb{I}-\Pi) \hat{P}^{k}+\text { remainder. }
\end{aligned}
$$

The remainder is estimated by

$$
C\left(\exp (-\omega t)+\int_{0}^{t} \frac{\exp \left(-\omega\left(t-t^{\prime}\right)+\delta_{k}(0) t^{\prime}\right)}{\sqrt{t-t^{\prime}}} d t^{\prime}\right) \leq C \exp \left(-\delta^{\prime} t\right),
$$

where $\delta^{\prime}$ is any strictly positive number which is strictly smaller than $\delta_{k}(0)$.

Let us consider now $\bar{B}^{\alpha_{1}} \partial^{\alpha_{2}} v^{j} / \partial s^{\alpha_{2}}$. As a consequence of the induction hypotheses, there exists a $\delta_{k}(\alpha)$ such that

$$
\left|\bar{B}^{\alpha_{1}} \frac{\partial^{\alpha_{2}} v^{j}}{\partial s^{\alpha_{2}}}\left(\hat{P}^{k}(., \sigma, s)-P^{k}(., \sigma, t, s)\right)\right|_{2} \leq C \exp \left(-t \delta_{k}(\alpha)\right),
$$

The constant $\delta_{k}(\alpha)$ is similar to the constant $\delta_{k}(0)$ : it is an minimum of a finite collection of $\gamma_{k}(\beta)$, where $j \leq k-1$ and $\beta$ runs in a finite set of multiindices 
depending upon $N-1$, the dimension of $\bar{\Gamma}$. As $\bar{B}^{\alpha_{1}} \partial^{\alpha_{2}} v^{j} / \partial s^{\alpha_{2}}$ commutes with $A$, for all $\alpha$, we may write

$$
\left(\frac{\partial}{\partial t}+A\right) \bar{B}^{\alpha_{1}} \frac{\partial^{\alpha_{2}} v^{j}}{\partial s^{\alpha_{2}}}=\bar{B}^{\alpha_{1}} \frac{\partial^{\alpha_{2}} v^{j}}{\partial s^{\alpha_{2}}} P^{k}
$$

and apply the same arguments as above. This proves the desired estimates.

4.2. Pointwise estimates of $v^{k}$ and its derivatives. In this section, we prove the following result:

Theorem 4.2. For all $j \geq 0$, and all $\alpha=\left(\alpha_{1}, \alpha_{2}\right) \in \mathbb{N}^{2}$, there exist constants $\gamma_{j}^{\prime}(\alpha)>0$ and $C_{j}(\alpha)$ such that for all $(\mu, \sigma, t, s) \in \mathbb{R} \times \bar{\Gamma} \times \mathbb{R}^{+} \times\left[0, T^{*}\right]$ the following inequality holds

$$
\left|\bar{B}^{\alpha_{1}} \frac{\partial^{\alpha_{2}} v^{j}}{\partial s^{\alpha_{2}}}(\mu, \sigma, t, s)\right|+\left|\frac{\bar{B}^{\alpha_{1}} \partial^{\alpha_{2}+1} v^{j}}{\partial s^{\alpha_{2}} \partial \mu}(\mu, \sigma, t, s)\right| \leq C_{j}(\alpha) \exp \left(-\gamma_{j}^{\prime}(\alpha)|\mu|\right) .
$$

The proof is in several steps, and uses precise analytical results on the fundamental solution of

$$
\frac{\partial v}{\partial t}-\frac{\partial^{2} v}{\partial x^{2}}-Z(x) v=0
$$

where $Z$ is a bounded function over $\mathbb{R}$. They look very much like the results of Friedman [Fr] who, however, does not prove estimates in unbounded domains for large times. We recall the notation

$$
\mathscr{E}(x, t)=\frac{1}{\sqrt{4 \pi t}} \exp \left(-\frac{|x|^{2}}{4 t}\right)
$$

for the fundamental solution of the heat equation, and we observe that the fundamental solution of (4.15) is invariant by translations in time.

Lemma 4.3. Let $Z$ be a bounded function over $\mathbb{R}$, and let $\mathscr{F}(x, y, t)$ be the fundamental solution of (4.15). Then

$$
|\mathscr{F}(x, y, t)| \leq \mathscr{E}(x-y, t) \exp \left(t|Z|_{\infty}\right),
$$

and for all $\kappa>0$, there exists a constant $K$ such that

$$
\left|\frac{\partial \mathscr{F}}{\partial x}\right| \leq \frac{K}{\sqrt{t}} \mathscr{E}(x-y, t(1+\kappa)) \exp \left(t\left(|Z|_{\infty}+\kappa\right)\right) .
$$

Proof. We proceed along the lines of [Fr, Chapter 1], and use the parametrix method of E. E. Levi: we look for an $\mathscr{F}$ of the form

$$
\mathscr{F}(x, y, t)=\mathscr{E}(x,-y, t)+\int_{0}^{t} \int \mathscr{E}(x-\eta, t-\tau) \Psi(\eta, y, \tau) d \eta d \tau .
$$

The fundamental solution satisfies (4.15), with $y$ as a parameter. According to [Fr] and the functional justification given therein, $\Psi$ satisfies the integral equation

$$
\Psi(x, y, t)=Z(x) \mathscr{E}(x-y, t)+\int_{0}^{t} \int Z(x) \mathscr{E}(x-\eta, t-\tau) \Psi(\eta, y, \tau) d \eta d \tau .
$$


Let

$$
\left\{\begin{array}{l}
\Psi^{0}(x, y, t)=Z(x) \mathscr{E}(x-y, t), \\
\Psi_{k+1}(x, y, t)=\int_{0}^{t} \int Z(x) \mathscr{E}(x-\eta, t-\tau) \Psi_{k}(\eta, y, \tau) d \eta d \tau .
\end{array}\right.
$$

It is proved in $[\mathrm{Fr}]$ that the series

$$
\Psi(x, y, t)=\sum_{k \geq 0} \Psi_{k}(x, y, t)
$$

converges and estimates on bounded time intervals are given therein. For later purposes, we have to know precisely how these estimates increase in time. The following inequality holds

$$
\left|\Psi_{k}(x, y, t)\right| \leq|Z|_{\infty}^{k+1} \mathscr{E}(x-y, t) \frac{t^{k}}{k !} .
$$

This relation is clear for $k=0$; assume that it is true at order $k$; then, at order $k+1$,

(4.22) $\left|\Psi_{k+1}(x, y, t)\right| \leq \int_{0}^{t} \int|Z(x)| \mathscr{E}(x-\eta, t-\tau)|Z|_{\infty}^{k+1} \mathscr{E}(\eta-y, \tau) \frac{t^{k}}{k !} d \eta d \tau$.

The following identity expresses the semigroup property of the heat equation:

$$
\int \mathscr{E}\left(x-\eta, t_{1}\right) \mathscr{E}\left(\eta-y, t_{2}\right) d \eta=\mathscr{E}\left(x-\eta, t_{1}+t_{2}\right), \quad \forall t_{1}, t_{2} \geq 0, \forall x, y \in \mathbb{R} .
$$

Therefore, the right-hand side of (4.22) is estimated by

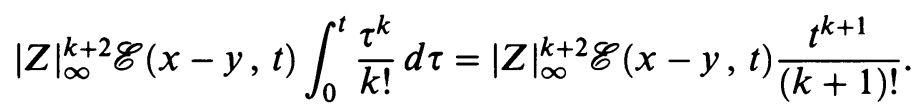

This proves (4.22). From (4.21) we deduce that

$$
|\Psi(x, y, t)| \leq|Z|_{\infty} \exp \left(t|Z|_{\infty}\right) \mathscr{E}(x-y, t) .
$$

This relation and (4.18) imply the first estimate (4.16).

To prove the second estimate, we observe that for all $\kappa>0$, there exists a constant $K$ such that

$$
\left|\frac{\partial \mathscr{E}}{\partial x}(x, t)\right| \leq \frac{K}{\sqrt{t}} \mathscr{E}(x,(1+\kappa) t) .
$$

We differentiate (4.18) with respect to $x$ :

(4.25) $\frac{\partial \mathscr{F}}{\partial x}(x, y, t)=\frac{\partial \mathscr{E}}{\partial x}(x-y, t)+\int_{0}^{t} \int \frac{\partial \mathscr{E}}{\partial x}(x-\eta, t-\tau) \Psi(\eta, y, \tau) d \eta d \tau$.

Therefore, to obtain an estimate over $\partial_{x} \mathscr{F}$, it suffices to estimate

$$
\int_{0}^{t} \int\left|\frac{\partial \mathscr{E}}{\partial x}(x-\eta, t-\tau)\right|\left|\Psi^{k}(\eta, y, \tau)\right| d \eta d \tau
$$

From (4.22), (4.23), and (4.24), we can see that (4.26) is estimated by

$$
\mathscr{E}(x-y,(1+\kappa) t) K \sqrt{1+\kappa}|Z|_{\infty}^{k+1} \int_{0}^{t} \frac{\tau^{k}}{k ! \sqrt{t-\tau}} d \tau .
$$


We calculate the above time integral using the Euler functions $\mathbf{B}$ and $\boldsymbol{\Gamma}$ :

$$
\int_{0}^{t} \frac{\tau^{k}}{k ! \sqrt{t-\tau}} d \tau=\frac{t^{k+1 / 2}}{k !} \int_{0}^{1} \frac{t^{\prime k}}{\sqrt{1-t^{\prime}}} d t^{\prime}=\frac{t^{k+1 / 2} \mathbf{B}(1 / 2, k+1)}{k !}=\frac{t^{k+1 / 2} \Gamma(1 / 2)}{\Gamma(k+3 / 2)} \text {. }
$$

We obtain a first estimate for $\partial_{x} \mathscr{F}$ :

$$
\left|\frac{\partial \mathscr{F}(x, y, t)}{\partial x}\right| \leq \frac{K(1+\kappa)}{\sqrt{t}} \mathscr{E}(x-y,(1+\kappa) t)\left(1+\Gamma(1 / 2) \sum_{k \geq 0} \frac{\left(t|Z|_{\infty}\right)^{k+1}}{\Gamma(k+3 / 2)}\right) \text {. }
$$

Consider the function

$$
g(z)=1+\sum_{k=1}^{\infty} \frac{\Gamma(1 / 2) z^{k}}{\Gamma(k+1 / 2)}
$$

Stirling's formula implies that

$$
\frac{\Gamma(k+1)}{\Gamma(k+1 / 2)} \sim k^{1 / 2} \text { as } k \rightarrow \infty .
$$

Thus, for all $\kappa$, there is a constant $K^{\prime} \geq 1$ such that

$$
\frac{\Gamma(1 / 2) \Gamma(k+1)}{\Gamma(k+1 / 2)} \leq K^{\prime}(1+\kappa)^{k}, \quad \forall k \geq 1 .
$$

Hence, the estimate relative to $g$ :

$$
|g(z)| \leq K^{\prime} \exp ((1+\kappa)|z|), \quad \forall z \in \mathbf{C},
$$

and therefore

$$
\left|\frac{\partial \mathscr{F}(x, y, t)}{\partial x}\right| \leq \frac{K \sqrt{1+\kappa}}{\sqrt{t}} \mathscr{E}(x-y,(1+\kappa) t) K^{\prime} \exp \left(t|Z|_{\infty}(1+\kappa)\right),
$$

which concludes the proof.

We prove now a useful corollary:

Corollary 4.4. Let $V$ be a bounded function over $\mathbb{R}$; assume that there exists a strictly positive number $L$ such that $V(x) \geq L, \forall x \in \mathbb{R}$. Then, the fundamental solution $\mathscr{G}(x, y, t)$ of

$$
\left(\partial_{t}-\partial_{x x}+V\right) u=0
$$

satisfies the estimate

$$
|\mathscr{G}(x, y, t)| \leq \exp (-L t) \mathscr{E}(x-y, t) .
$$

Moreover for all $\kappa>0$, there exists a $K$ such that

$$
\left|\frac{\partial \mathscr{G}}{\partial x}(x, y, t)\right| \leq \frac{K}{\sqrt{t}} \exp (-(L-q \kappa) t) \mathscr{E}(x-y, t(1+\kappa)),
$$

where $q=\left(|V|_{\infty}-L\right) / 2 L$.

Proof. Let

$$
L^{\prime}=\frac{L+|V|_{\infty}}{2}
$$

and let

$$
\mathscr{F}(x, y, t)=\mathscr{G}(x, y, t) \exp \left(L^{\prime} t\right) .
$$


Then, $\mathscr{F}$ is the fundamental solution of

$$
\left(\partial_{t}-\partial_{x x}+V-L^{\prime}\right) v=0
$$

to which we may apply Lemma 4.3:

$$
\begin{gathered}
|\mathscr{F}(x, y, t)| \leq \mathscr{E}(x-y, t) \exp \left(t\left|V-L^{\prime}\right|_{\infty}\right) \\
\left|\frac{\partial \mathscr{F}}{\partial x}\right| \leq \frac{K}{\sqrt{t}} \mathscr{E}(x-y, t(1+\kappa)) \exp \left(t(1+\kappa)\left|V-L^{\prime}\right|_{\infty}\right) .
\end{gathered}
$$

With our choice of $L^{\prime},\left|V-L^{\prime}\right|_{\infty}-L^{\prime}=-L$, and going back to $\mathscr{G}$, we obtain the desired estimate.

The next lemma gives sufficient conditions for exponential decrease with respect to space of solutions of the parabolic equation

$$
v_{t}+A v=f, \quad v(0)=0 .
$$

Lemma 4.5. Let us fix $L=\phi^{\prime}(1) / 3$, and assume that there exists $\gamma \in(0, \sqrt{L})$ such that

$$
|f(x, t)| \leq e^{-\gamma|x|}, \quad \forall x \in \mathbb{R}, \forall t \in \mathbb{R}^{+},
$$

and that the solution of (4.31) satisfies the estimate

$$
\int|v(x, t)|^{2} d x \leq 1, \quad \forall t \in \mathbb{R}^{+} .
$$

Then, for all $\kappa>0$, there exists a constant $C$ such that

$$
|v(x, t)|+\left|v_{x}(x, t)\right| \leq C \exp (-(\gamma-\kappa)|x|) .
$$

Proof. Let $W=\phi^{\prime}(\theta)$; we rewrite (4.31) as

$$
v_{t}-v_{x x}+(W-L)_{+} v+L v=f+(W-L)_{-} v .
$$

If we let $V=L+(W-L)_{+}$, we may apply Corollary 4.4 to $v$, and we obtain

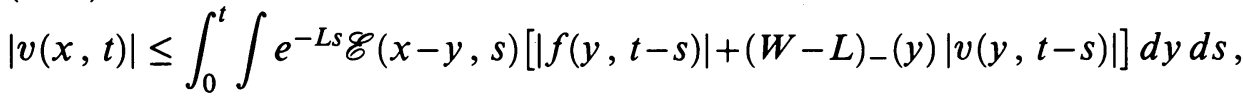
and

$$
\begin{array}{r}
\left|v_{x}(x, t)\right| \leq K \int_{0}^{t} \int \frac{1}{\sqrt{s}} e^{-(L(1-\kappa)) s} \mathscr{E}(x-y, s(1+\kappa)) \\
\quad \times\left[|f(y, t-s)|+(W-L)_{-}(y)|v(y, t-s)|\right] d y d s .
\end{array}
$$

We shall analyze (4.35) and (4.36) together. Denote $s^{\prime}=s(1+\kappa)$, with $\kappa \geq 0$. From our assumptions,

$$
\int \mathscr{E}\left(x-y, s^{\prime}\right)|f(y, t-s)| d y \leq \int \mathscr{E}\left(x-y, s^{\prime}\right) e^{-\gamma|y|} d y .
$$

Let $z$ be a solution of

$$
\begin{aligned}
& z_{t}-z_{x x}=0, \quad x \in \mathbb{R}, t \geq 0, \\
& z(x, 0)=e^{-\gamma|x|}, \quad x \in \mathbb{R} .
\end{aligned}
$$


A simple calculation shows that

$$
\frac{\exp \left(L^{\prime} t-\gamma|x|\right)}{L^{\prime}-\gamma^{2}}
$$

is a supersolution for (4.37), provided that $L^{\prime}>\gamma^{2}$. Therefore,

Hence, if $L^{\prime}<L$,

$$
\int \mathscr{E}\left(x-y, s^{\prime}\right) e^{-\gamma|y|} d y \leq \frac{\exp \left(L^{\prime} s^{\prime}-\gamma|x|\right)}{L^{\prime}-\gamma^{2}}
$$

$$
\int_{0}^{t} \int e^{-L s} \mathscr{E}(x-y, s)|f(y, t-s)| d y d s \leq C e^{-\gamma|x|} .
$$

Similarly,

$$
\begin{gathered}
\int_{0}^{t} \int e^{-(L(1-\kappa)) s} s^{-1 / 2} \mathscr{E}(x-y, s(1+\kappa))|f(y, t-s)| d y d s \\
\quad \leq \int_{0}^{t} \frac{\exp \left(L^{\prime}(1+\kappa) s-\gamma|x|-L(1-\kappa) s\right)}{\sqrt{s}\left(L^{\prime}-\gamma^{2}\right)} d s \leq C e^{-\gamma|x|},
\end{gathered}
$$

if $L^{\prime}(1+\kappa)<L(1-\kappa)$.

We turn to the piece of the integral in (4.36) involving $v$ : with $X>0$ such that $[-X, X]$ contains the (bounded) support of $(W-L)_{-}$, by Cauchy-Schwarz inequality and our assumptions we obtain

$$
\begin{aligned}
& \int \mathscr{E}\left(x-y, s^{\prime}\right)(W-L)_{-}(y)|v(y, t-s)| d y \\
& \leq\left(4 \pi s^{\prime}\right)^{1 / 2}\left(\int_{-X}^{X} \exp \left(-|x-y|^{2} / 2 s^{\prime}\right) d y\right)^{1 / 2} .
\end{aligned}
$$

We have two cases:

$$
\begin{aligned}
& \left(\int_{-X}^{X} \exp \left(-|x-y|^{2} / 2 s^{\prime}\right) d y\right)^{1 / 2} \\
& \quad \leq \begin{cases}\sqrt{2 X} \exp \left(-(|x|-X)^{2} / 4 s^{\prime}\right) & \text { if }|x| \geq X+1, \\
\left(4 \pi s^{\prime}\right)^{1 / 4} & \text { if }|x| \leq X+1 .\end{cases}
\end{aligned}
$$

Consider the first case. If $|x| \geq X+1$ and $\alpha=0$ or $-1 / 2$,

$$
\begin{gathered}
\int_{0}^{t} \int \mathscr{E}\left(x-y, s^{\prime}\right)(W-L)_{-}(y)|v(y, t-s)| s^{\alpha} e^{-(L(1-\kappa)) s} d y d s \\
\leq C \int_{0}^{t} \exp \left(-\frac{(|x|-X)^{2}}{4 s(1+\kappa)}-(L(1-\kappa)) s\right) \frac{s^{\alpha-1 / 2}}{\sqrt{1+\kappa}} d s .
\end{gathered}
$$

Let

$$
s^{*}=\frac{|x|-X}{2 \sqrt{(L(1-\kappa))(1+\kappa)}} .
$$

Then, with the change of variable $s=s^{*} r$,

$$
\begin{gathered}
\int_{0}^{t} \exp \left(-\frac{(|x|-X)^{2}}{4 s(1+\kappa)}-(L(1-\kappa)) s\right) \frac{s^{\alpha-1 / 2}}{\sqrt{1+\kappa}} d s \\
\leq \frac{\left(s^{*}\right)^{\alpha+1 / 2}}{\sqrt{1+\kappa}} \int_{0}^{\infty} r^{\alpha-1 / 2} \exp \left[-y^{*}\left(r+\frac{1}{r}\right)\right] d r
\end{gathered}
$$


where

$$
y^{*}=\frac{1}{2}(|x|-X) \sqrt{\frac{L(1-\kappa)}{1+\kappa}} .
$$

By asymptotics for Laplace integrals, as $y^{*}$ tends to infinity

$$
\int_{0}^{\infty} r^{\alpha-1 / 2} \exp \left[-y^{*}\left(r+\frac{1}{r}\right)\right] d r \sim \sqrt{\frac{\pi}{y^{*}}} \exp \left(-2 y^{*}\right) .
$$

In particular, for all small enough $\kappa$, there exists a constant $C$ such that for $|x| \geq X+1$

$$
\begin{gathered}
\int_{0}^{t} \exp \left(-\frac{(|x|-X)^{2}}{4 s(1+\kappa)}-(L(1-\kappa)) s\right) \frac{s^{\alpha-1 / 2}}{\sqrt{1+\kappa}} d s \\
\leq C \exp \left(-(|x|-X) \sqrt{\frac{L(1-\kappa)}{1+\kappa}}\right)
\end{gathered}
$$

we recall that $\alpha \leq 0$.

In the second case $(|x| \leq X+1)$, we can see that

$$
\int_{0}^{\infty} \exp (-(L(1-\kappa)) s) s^{\alpha-1 / 4} d s \leq C
$$

where $C$ is bounded for all small enough $\kappa$.

If we put together (4.40) and (4.41), we can see that

$$
\begin{gathered}
\int_{0}^{t} \int \mathscr{E}(x-y, s)(W-L)_{-}(y)|v(y, t-s)| e^{-L s} d y d s \\
\leq C \exp (-|x| \sqrt{L}), \quad \forall x \in \mathbb{R}, \forall t \in \mathbb{R}^{+},
\end{gathered}
$$

and

$$
\begin{gathered}
\int_{0}^{t} \int \mathscr{E}(x-y, s(1+\kappa))(W-L)_{-}(y)|v(y, t-s)| e^{-(L(1-\kappa)) s} s^{-1 / 2} d y d s \\
\leq C \exp \left(-(|x|-X) \sqrt{\frac{L(1-\kappa)}{1+\kappa}}\right), \quad \forall x \in \mathbb{R}, \forall t \in \mathbb{R}^{+},
\end{gathered}
$$

Putting together (4.38), (4.39), (4.42) and (4.43), we obtain (4.34), and the lemma is proved.

We may see that this lemma is of a general nature and is easily generalized to the $N$-dimensional case.

Proof of Theorem 4.2. The proof is by induction, and it is an immediate application of Lemma 4.5 and Lemma 4.1 to (4.13): indeed, $P^{0}=P^{1}=0$ and $P^{2}=C(-\sigma, s) \theta(\mu)$ (see (3.49)) where $\theta(\mu)$ decays exponentially by (1.6).

4.3. Remainder estimates. Let $q>0$ and define for $x \in \mathscr{V}^{0}(s)$

$$
\tilde{u}^{q, h}(x, t)=\sum_{j=0}^{q} h^{j} v^{j}\left(\frac{\Lambda^{q-1, h}\left(x, h^{2} t ; h\right)}{h}, \Sigma^{q-2, h}\left(x, h^{2} t ; h\right), h^{2} t, t\right)
$$

with

$$
\Lambda^{q, h}(x, s)=\sum_{j=0}^{q} h^{j} \Lambda^{j}(x, s), \quad \Sigma^{q, h}(x, s ; h)=\sum_{j=0}^{q} h^{j} \Sigma^{j}(x, s),
$$


where $v^{j}, \Lambda^{j}$ are determined according to the algorithm described in the previous section.

The remainder $\tilde{\rho}^{q, h}$ is defined by

$$
\tilde{\rho}^{q, h}=\left(\frac{\partial}{\partial t}-\Delta\right) \tilde{u}^{q, h}+\phi\left(\tilde{u}^{q, h}\right) .
$$

Lemma 4.6. For every $q \geq 1$, there exists a constant $C$ depending on $q$ and the initial data $\Gamma_{0}$ such that

$$
\max _{\mathscr{V}^{0}\left(h^{2} t\right)}\left|\tilde{\rho}^{q, h}(x, t)\right| \leq C h^{q+1} .
$$

and

$$
\left(\int_{\mathscr{V}^{0}(s)}\left|\tilde{\rho}^{q, h}\right|^{2} d x\right)^{1 / 2} \leq C h^{q+3 / 2} .
$$

Proof. Denote by $\Sigma^{q, h}$ the expansion of $\Sigma$ with $q$ terms, $X^{q, h}$ the inverse diffeomorphism, and $L^{q, h}$ the analogue of $L$, with $\Lambda$ and $X$ respectively replaced by $\Lambda^{q, h}$ and $X^{q, h}$. Clearly, $L^{q, h}$ coincides with the expansion of $L$ up to order $q$. Similarly, $B^{q, h}$ and $T^{q, h}$ are defined from $X^{q, h}$ and $\Lambda^{q, h}$.

Then,

$$
\begin{aligned}
\left(\frac{\partial}{\partial t}-\Delta\right) \tilde{u}^{q, h}=\sum_{j=0}^{q} h^{j}\left[\frac{\partial v^{j}}{\partial \mu}\right. & -\frac{\partial^{2} v^{j}}{\partial \mu^{2}}+h L^{q-1, h} \frac{\partial v^{j}}{\partial \mu} \\
& \left.+h^{2}\left(T^{q-1, h} \cdot v^{j}-B^{q-1, h} v^{j}+\frac{\partial v^{j}}{\partial s}\right)\right] \\
= & \sum_{j=0}^{q} h^{j}\left[\frac{\partial v^{j}}{\partial \mu}-\frac{\partial^{2} v^{j}}{\partial \mu^{2}}+h L^{q-j-1, h} \frac{\partial v^{j}}{\partial \mu}\right. \\
& \left.+h^{2}\left(T^{q-j-2, h} \cdot v^{j}-B^{q-j-2, h} v^{j}+\frac{\partial v^{j}}{\partial s}\right)\right] \\
& +O\left(h^{q+1}\right),
\end{aligned}
$$

where $O\left(h^{q+1}\right)$ is uniform in $s \in\left[0, T^{*}\right], h \in\left[0, h^{*}\right]$ and $x \in \mathscr{V}^{0}(s)$, because

$$
h^{j}\left(L^{q, h}-L^{q-j-1, h}\right) \frac{\partial v^{j}}{\partial \mu}=O\left(h^{q}\right)
$$

with similar estimates for the other differences; they are an immediate consequence of Theorem 4.1. It follows from (3.6) and (3.28) that

$$
\begin{aligned}
\left(\frac{\partial}{\partial t}-\Delta\right) \tilde{u}^{q, h}+\phi\left(\tilde{u}^{q, h}\right) & \\
= & -\left[\phi\left(v^{0}\right)+h^{2} \phi^{\prime}\left(v^{0}\right) v^{2}+\cdots+h^{q}\left(\phi^{\prime}\left(v^{0}\right) v^{q}+\Psi\left(v^{0}, \ldots, v^{q-1}\right)\right)\right] \\
& +\phi\left(\sum_{k=0}^{q} h^{k} v^{k}\right)+\left.O\left(h^{q+1}\right)\right|_{h=0} .
\end{aligned}
$$




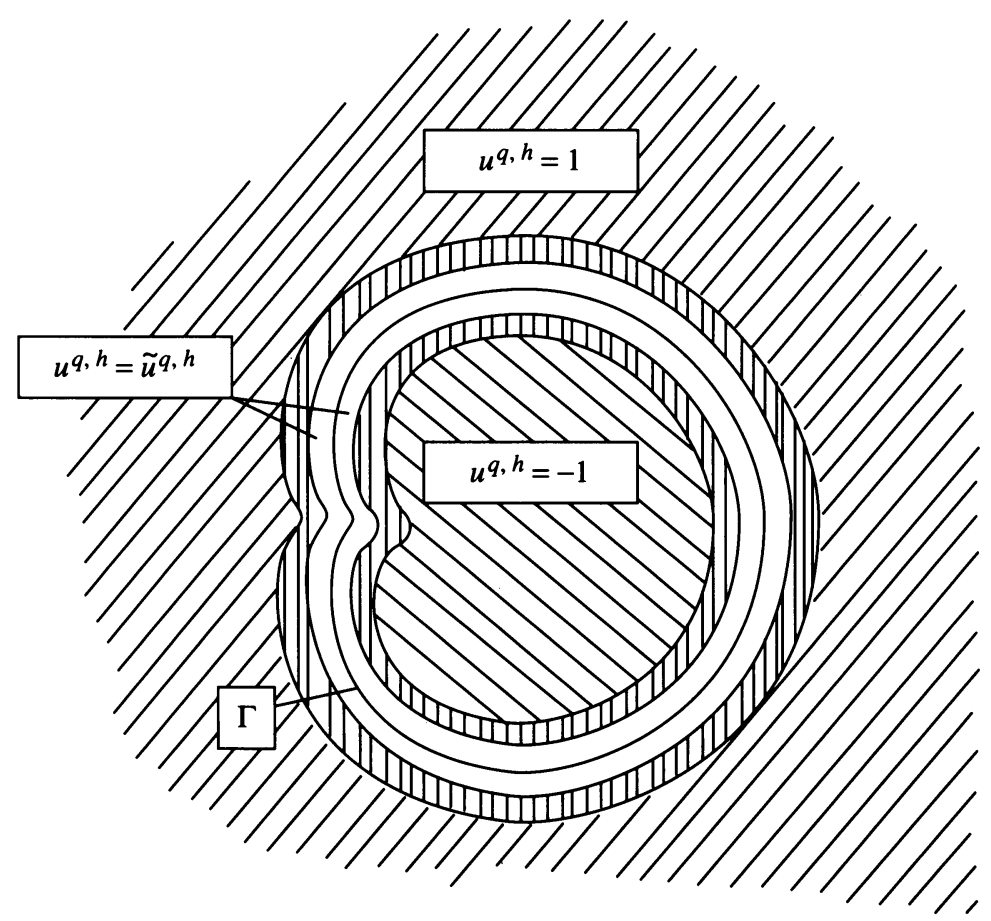

Figure 4.1. Patching $u^{q, h}$ with \pm 1 in a smooth fashion

By definition,

$$
\phi^{\prime}\left(v^{0}\right) v^{j}+\Psi^{j}\left(v^{0}, \ldots, v^{j-1}\right)=\left.j ! \frac{d^{j}}{d h^{j}} \phi\left(\sum_{k=0}^{q} h^{k} v^{k}\right)\right|_{h=0},
$$

and Taylor formula with integral remainder shows that

$$
\left(\frac{\partial}{\partial t}-\Delta\right) \tilde{u}^{q, h}+\phi\left(\tilde{u}^{q, h}\right)=O\left(h^{q+1}\right),
$$

where once again, $O\left(h^{q+1}\right)$ is uniform in $s \in\left[0, T^{*}\right], h \in\left[0, h^{*}\right]$ and $x \in$ $\mathscr{V}^{0}(s)$. Thus, we have obtained (4.45).

Let $J$ be the Jacobian of the transformation $(\lambda, \sigma) \mapsto X^{q, h}(\lambda, \sigma, s)$. Then, if $\tilde{\rho}_{1}^{q, h}$ denotes $\tilde{\rho}^{q, h}$ in coordinates $(\lambda, \sigma)$, we can write

$$
\begin{aligned}
\int_{\left|\Lambda^{q, h}(x, s)\right| \leq \bar{\lambda}}\left|\tilde{\rho}^{q, h}(x, s)\right|^{2} d x & =\int_{-\bar{\lambda} / h}^{\bar{\lambda} / h}\left|\tilde{\rho}_{1}^{q, h}(\mu, \sigma, s)\right|^{2}|J(\mu h, \sigma)| h d \mu d \sigma \\
& =O\left(h^{2 q+3}\right) .
\end{aligned}
$$

The conclusion is clear.

We now extend $\tilde{u}^{q, h}$ to all of $\mathbb{R}^{N}$ by using an appropriate partition of unity. Let $\omega$ be a smooth even function over $\mathbb{R}$ such that

$$
\omega(x)=\left\{\begin{array}{ll}
1 & \text { if }|x| \leq 1 / 2, \\
0 & \text { if }|x| \geq 1,
\end{array} \quad 0 \leq \omega(x)<1, \forall x \in \mathbb{R} .\right.
$$


We let

$$
u^{q, h}=\omega\left(\frac{\Lambda^{0}(x, s)}{h}\right) \tilde{u}^{q, h}(x, t)+\left(1-\omega\left(\frac{\Lambda^{0}(x, s)}{h}\right)\right) \operatorname{sgn}\left(\Lambda^{0}(x, s)\right) .
$$

The remainder relative to $u^{q, h}$ is

$$
\rho^{q, h}=\left(\frac{\partial}{\partial t}-\Delta\right) u^{q, h}+\phi\left(u^{q, h}\right) .
$$

For this global expansion in space, the following result holds:

Theorem 4.6. For every $q \geq 1$, there exists a constant $C$ depending on $q$, and the initial data $\Gamma_{0}$ such that

$$
\sup \left|\rho^{q, h}(x, t)\right| \leq C h^{q+1}
$$

and

$$
\left(\int\left|\rho^{q, h}\right|^{2} d x\right)^{1 / 2} \leq C h^{q+3 / 2} .
$$

Proof. It is enough to observe that the difference between $\omega \tilde{\rho}^{q, h}$ and $\rho^{q, h}$ contains derivatives of $v^{j}$, for $j \leq q-1$, which are at most of order 1 with respect to any of the variables $\mu, \sigma$ and $s$. Moreover, Theorem 4.2 shows that this difference has its support in a set where these derivatives are $O\left(e^{-C / h}\right)$. the conclusion is clear.

\section{Spectral estimates}

Throughout this section, the time dependence is understated. Let $\Gamma$ be a compact smooth manifold of dimension $N-1$ embedded in $\mathbb{R}^{N}$. We define the diffeomorphism $Y$, the normal coordinate $\Lambda$ and the tangential coordinate $S$ as in subsection 3.2; the latter are smooth on some tubular neighborhood $\mathscr{V}[\bar{\lambda}]$ of $\Gamma$, where for $r \in(0, \bar{\lambda}]$ we define $\mathscr{V}[r]$ of thickness $2 r$ by

$$
\mathscr{V}[r]=\left\{x \in \mathbb{R}^{N} /|\Lambda(x)| \leq r\right\} .
$$

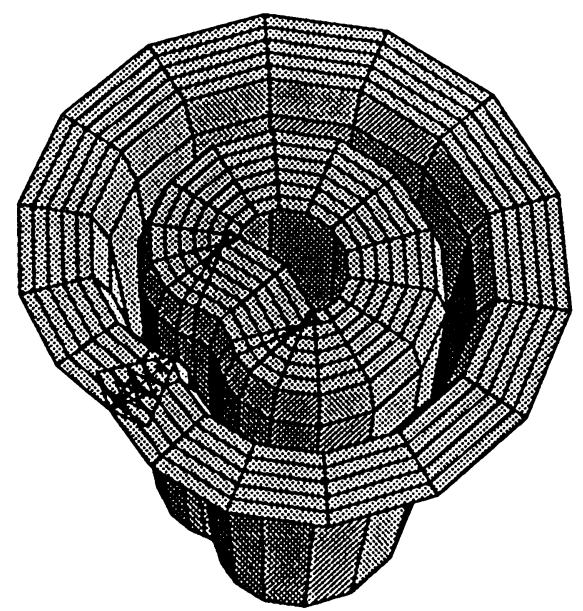

Figure 5.1. The potential $V^{h}$ in a neighbourhood of $\Gamma$ 
Define a potential

$$
V^{h}(x)=\phi^{\prime}\left(u^{0, h}(x)\right)
$$

where $u^{q, h}$ has been defined at (4.50). Let $\mathscr{A}^{h}$ be the selfadjoint operator in $L^{2}\left(\mathbb{R}^{N}\right)$ determined by

$$
D\left(\mathscr{A}^{h}\right)=H^{2}\left(\mathbb{R}^{N}\right) ; \quad \mathscr{A}^{h} v=-h^{2} \Delta v+V^{h} v .
$$

The lower bound of the spectrum of $\mathscr{A}^{h}$ is denoted $\chi^{h}$. In this section, we prove the following result:

Theorem 5.1. There exist nonnegative constants $C$ and $h^{*}$ depending only on $\Gamma$ such that the lower bound of the spectrum of $\mathscr{A}^{h}$ is larger than or equal to $-\mathrm{Ch}^{2}$, for all $h \leq h^{*}$. The same result holds true if one replaces, in the definition of the potential $V^{h}$, the function $u^{0, h}$ with any patched higher order approximation $u^{q, h}, q>0$.

5.1. Strategy of proof and first bounds. The proof of Theorem 5.1 takes all the present section. Let us sketch its strategy. We associate to $\mathscr{A}^{h}$ a Rayleigh quotient, defined for $y \in H^{1}\left(\mathbb{R}^{N}\right)$ and $y \neq 0$ :

$$
R^{h}[y]=\frac{\int\left\{h^{2}|\nabla y|^{2}+V^{h} y^{2}\right\} d x}{\int y^{2} d x} .
$$

According to general results,

$$
\chi^{h}=\inf _{y \neq 0} R^{h}[y]
$$

This $\chi^{h}$ is an eigenvalue of $\mathscr{A}^{h}$ (see below), but it is not isolated in the spectrum of $\mathscr{A}^{h}$ uniformly with respect to $h$ because the tangential modes of $\mathscr{A}^{h}$ are only an $O\left(h^{2}\right)$ apart. We compare the Rayleigh quotient $R^{h}$ over $\mathbb{R}^{N}$ to the Rayleigh quotient of the restriction of $\mathscr{A}^{h}$ to the thin neighborhood $\mathscr{V}[\sqrt{h}]$ with Neumann conditions. The comparison is made possible by the estimates of subsection 5.2. The eigenvalue problem for $\left.\mathscr{A}^{h}\right|_{\mathscr{V}[\sqrt{h}]}$ with Neumann conditions is almost decoupled in the tangential and normal variables; moreover, to obtain a lower bound, we restrict ourselves to the normal modes, that is to a family of one-dimensional Neumann problems on $[-\sqrt{h}, \sqrt{h}]$. A stretching of variables leads to a family of Neumann problems which are very close to

$$
-\frac{\partial^{2} z}{\partial \mu^{2}}+\phi^{\prime}(\boldsymbol{\theta}) z=\lambda z
$$

on the line (subsection 5.3). This allows to show that the lowest eigenvalue of the one-dimensional Neumann problem is isolated uniformly in $h$. To conclude, we give an approximate eigenvalue of this one dimensional Neumann problem to $O\left(h^{2}\right)$, we apply a classical perturbation result for eigenvalues of selfadjoint operators and we take an infimum over $\Gamma$ of the bounds of the spectra of all normal operators.

Let $\omega$ be as in (4.49), and define

$$
\Theta^{h}(\mu)=\omega(h \mu / \bar{\lambda}) \Theta(\mu)+[1-\omega(h \mu / \bar{\lambda})] \operatorname{sgn} \mu .
$$

Then

$$
u^{0, h}(x)=\Theta^{h}\left(\frac{\Lambda^{0}(x)}{h}\right) .
$$




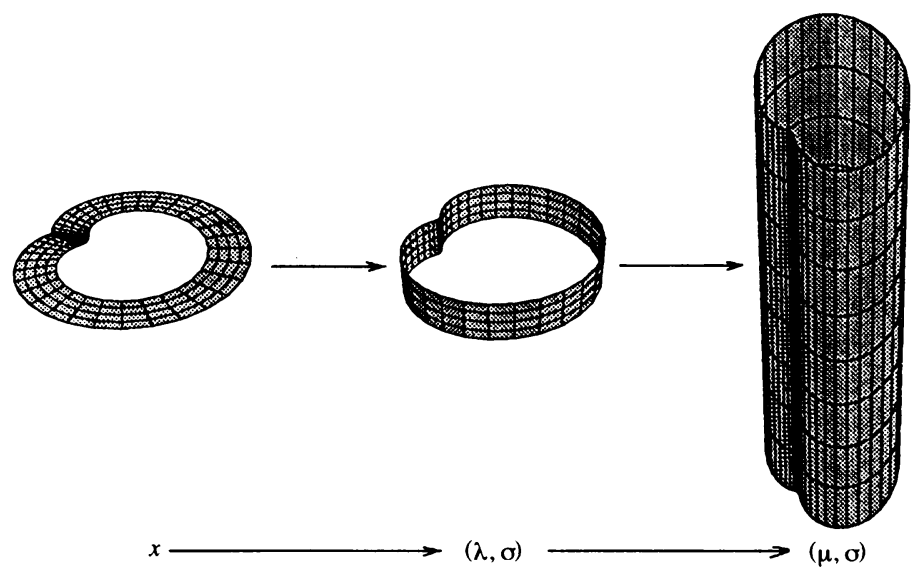

FIGURE 5.2. Change of variables

It is convenient to introduce

$$
\theta^{h}=\frac{d \Theta^{h}}{d \mu}
$$

We start with some very simple bounds on $\chi^{h}$

Lemma 5.2. Let $\chi^{h}$ be the lower bound of the spectrum of $\mathscr{A}^{h}$. Then,

$$
\limsup _{h \rightarrow 0} \chi^{h} \leq 0 .
$$

Moreover, $\chi^{h}$ is a simple eigenvalue of $\mathscr{A}^{h}$, the corresponding eigenspace is spanned by a nonnegative normalized eigenfunction $\zeta^{h}$, and

$$
\forall h>0, \quad \chi^{h}>\phi^{\prime}(0) \text {. }
$$

Proof. We know that the lower bound of the spectrum of $\mathscr{A}^{h}$ is given by (5.5). To prove (5.8) it is sufficient to exhibit a function $y^{h} \in H^{1}\left(\mathbb{R}^{N}\right)$ such that

$$
\lim _{h \rightarrow 0} R^{h}\left[y^{h}\right]=0 .
$$

Let $y^{h}(x)=\theta^{h}(\Lambda(x) / h)$. In the numerator of the Rayleigh quotient we perform the successive changes of variables

$$
x \mapsto(\lambda=\Lambda(x), \sigma=S(x)) ; \quad(\lambda, \sigma) \mapsto\left(\mu=\frac{\lambda}{h}, \sigma\right) .
$$

The Jacobian of the first change of variables is denoted $J(\lambda, \sigma)$; we obtain

$$
\begin{aligned}
\int\left[h^{2}\left|\nabla y^{h}(x)\right|^{2}+V^{h}(x)\left|y^{h}(x)\right|^{2}\right] d x \\
\quad=h \int_{\Gamma} \int_{-\bar{\lambda} / h}^{\bar{\lambda} / h}\left[\left|\frac{d \theta^{h}}{d \mu}(\mu)\right|^{2}+\phi^{\prime}\left(\Theta^{h}(\mu)\right)\left|\theta^{h}(\mu)\right|^{2}\right] J(h \mu, \sigma) d \mu d \sigma .
\end{aligned}
$$

For $h=0, \Theta^{h}=\Theta$. Relation (1.19) and an integration by parts show that

$$
\int\left[\theta^{\prime}(\mu)^{2}+\phi^{\prime}(\Theta(\mu)) \theta(\mu)^{2}\right] d \mu=\int\left[-\theta^{\prime \prime}(\mu)+\phi^{\prime}(\Theta(\mu)) \theta(\mu)\right] \theta(\mu) d \mu=0 .
$$


Hence, the limit as $h$ tends to zero of the factor of $h$ in the right hand side of (5.11) vanishes, and the numerator of $R^{h}\left[y^{h}\right]$ is $o(h)$. On the other hand, the denominator of $R^{h}\left[y^{h}\right]$, after the changes of variable (5.10) becomes

$$
h \int_{\Gamma} \int_{-\bar{\lambda} / h}^{\bar{\lambda} / h}\left|\theta^{h}(\mu)\right|^{2} J(h \mu, \sigma) d \mu d \sigma .
$$

As $h$ tends to zero, (5.12) is equivalent to

$$
h \int_{\mathbb{R}} \theta(\mu)^{2} d \mu \int_{\Gamma} J(0, \sigma) d \sigma .
$$

Therefore, (5.8) is proved.

Assumptions (1.1) imply that $\phi^{\prime}$ is increasing over $\mathbb{R}^{+}$. In particular, $\phi^{\prime}(0) \leq$ $V^{h}$, which implies immediately that $\chi^{h} \geq \phi^{\prime}(0)$. The limit as $|x|$ tends to infinity of $V^{h}(x)$ is $\phi^{\prime}( \pm 1)>0$, according to the assumptions on $\phi$. We know from [Si] that the intersection of the spectrum of $\mathscr{A}^{h}$ with $\left(-\infty, \phi^{\prime}(1)\right)$ consists only of isolated eigenvalues. For small enough $h,(5.8)$ implies $\chi^{h}<\phi^{\prime}(1)$, thus $\chi^{h}$ is an eigenvalue; let $\zeta^{h}$ be a normalized eigenfunction associated to the eigenvalue $\chi^{h}$. It follows from the Krein-Rutman theorem that $\zeta^{h}$ does not change sign, and that $\chi^{h}$ is simple. Assume that $\chi^{h}=\phi^{\prime}(0)$; then

$$
\int\left[h^{2}\left|\nabla \zeta^{h}(x)\right|^{2}+\left(V^{h}(x)-\phi^{\prime}(0)\right)\left|\zeta^{h}(x)\right|^{2}\right] d x=0
$$

which implies $\zeta^{h}=0$. We have a contradiction.

5.2. Estimates away from the interface. In this subsection, we prove the following result

Lemma 5.3. There exist constants $k$ and $k^{\prime}$ such that for all small enough $h$

$$
\int_{\mathbf{R}^{N} \backslash \mathscr{V}[\sqrt{h}]}\left[h^{2}\left|\nabla \zeta^{h}\right|^{2}+\left|\zeta^{h}\right|^{2}\right] d x \leq k^{\prime} e^{-k / \sqrt{h}} .
$$

Proof. Let $\bar{V}=\phi^{\prime}(1)>0$; we can rewrite the equation satisfied by the eigenvector $\zeta^{h}$

$$
-h^{2} \Delta \zeta^{h}+V^{h} \zeta^{h}=\chi^{h} \zeta^{h}
$$

$$
-h^{2} \Delta \zeta^{h}+\left(\bar{V}-\chi^{h}\right) \zeta^{h}=\left(\bar{V}-V^{h}\right) \zeta^{h} .
$$

If $\delta^{h}$ is the characteristic function of $\mathscr{V}[\sqrt{h} / 2]$, we define

$$
f_{1}=\left(\bar{V}-V^{h}\right) \delta^{h} \zeta^{h} ; \quad f_{2}=\left(\bar{V}-V^{h}\right)\left(1-\delta^{h}\right) \zeta^{h} .
$$

Then, $\chi^{h}=v_{1}+v_{2}$ where $v_{j}(j=1,2)$ is the unique solution of the problem

$$
-h^{2} \Delta v_{j}+\left(\bar{V}-\chi^{h}\right) v_{j}=f_{j} \text {. }
$$

We know that there exists a constant $C$ such that

$$
\left|\bar{V}-V^{h}(x)\right| \leq C \exp (-\gamma|\Lambda(x)| / h) .
$$

This implies that

$$
\left|f_{2}\right|_{\infty} \leq C \exp (-\gamma / 2 \sqrt{h}) .
$$


Therefore, if we multiply (5.16) for $j=2$ by $v_{2}$, and integrate, we obtain

$$
\int\left(h^{2}\left|\nabla v_{2}\right|^{2}+\left(\bar{V}-\chi^{h}\right)\left|v_{2}\right|^{2}\right) d x \leq C \exp (-\gamma / 2 \sqrt{h})\left|v_{2}\right|_{2},
$$

because $\zeta^{h}$ is normalized. Since there exists a number $\alpha \in(0,1)$ such that for all $h$

$$
\bar{V}-\chi^{h} \geq \alpha
$$

we can see that

$$
\left|v_{2}\right|_{2} \leq \alpha^{-1} C \exp (-\gamma / 2 \sqrt{h}) .
$$

Thus, we can conclude that

$$
\int\left(h^{2}\left|\nabla v_{2}\right|^{2}+\left|v_{2}\right|^{2}\right) d x \leq \alpha^{-2} C^{2} \exp (-\gamma / \sqrt{h}) .
$$

Let us now estimate $v_{1}$ on the complement of $\mathscr{V}[\sqrt{h}]$. For this purpose, we use the Green function $G$ of $-\Delta+\mu^{2}$ which is defined by

$$
-\Delta G(x, \mu)+\mu^{2} G(x, \mu)=\delta \quad(\text { the Dirac mass at } 0)
$$

for $\mu$ a strictly positive number. For all positive $\mu$, there exists a $\kappa(\mu)$ which depends continuously on $\mu$ such that

$$
\max (|G(x, \mu)|,|\nabla G(x, \mu)|) \leq \kappa(\mu) e^{-\mu|x|} .
$$

Let

$$
\mu^{h}=\sqrt{\bar{V}-\chi^{h}} .
$$

Since $\chi^{h}$ is bounded from above by $\bar{V}-\alpha$ and from below by $\phi^{\prime}(0), G\left(., \mu^{h}\right)$ satisfies the following estimate uniformly in $h$ :

$$
\begin{array}{r}
\max \left(\left|G\left(x, \mu^{h}\right)\right|,\left|\nabla G\left(x, \mu^{h}\right)\right|\right) \leq \bar{\kappa} \exp \left(-|x| \mu^{h}\right), \\
\forall x \text { such that }|x| \geq 1 .
\end{array}
$$

We have

$$
v_{1}(x)=h^{-N} \int G\left(\frac{x-y}{h}, \mu^{h}\right) \delta^{h}(y)\left(\bar{V}-V^{h}\right)(y) \zeta^{h}(y) d y
$$

Since $\zeta^{h}$ is normalized, we have for all $m$ the estimate

$$
\left(\sum_{|\alpha|=m} \int\left|D^{\alpha} \zeta^{h}\right|^{2} d x\right)^{1 / 2} \leq C_{m} h^{-m} .
$$

This is obtained by applying $D^{\alpha}$ for $|\alpha|=m-1$ to (5.14), and by scalarly multiplying the result by $D^{\alpha} \zeta^{h}$.

Let us get now an estimate of $\left|\zeta^{h}\right|_{\infty}:$ if $m$ is such that $H^{m}\left(\mathbb{R}^{N}\right)$ is continuously injected into $L^{\infty}\left(\mathbb{R}^{N}\right)$, we let

$$
\sum_{|\alpha|=m} \int\left|D^{\alpha} \zeta^{h}\right|^{2} d x=a^{2}
$$

and we observe that if the function $f$ is defined by its Fourier transform

$$
\hat{f}(\xi)=\hat{\zeta}^{h}(\xi)\left(a^{2}+|\xi|^{2 m}\right)
$$


and $g$ is defined by

$$
\hat{g}(\xi)=\frac{1}{a^{2}+|\xi|^{2 m}},
$$

we have $\left|\zeta^{h}\right|_{\infty} \leq|f|_{2}|g|_{2}$. It is clear that $|f|_{2} \leq C a$ and a simple calculation shows that $|g|_{2} \leq C a^{-1+(N / 2 m)}$. Therefore, we have from the definition of $a$ $|\zeta|_{\infty} \leq C h^{-N / 2}$, and we can deduce from (5.18) that

$$
\left|v_{1}(x)\right| \leq C h^{-3 N / 2} \int_{\mathscr{V}[\sqrt{h} / 2]}\left|G\left(\frac{x-y}{h}, \mu^{h}\right)\right| d y .
$$

Since we are interested only in $|\Lambda(x)| \geq \sqrt{h}$, we may apply estimate (5.16) to this integral, and we obtain

$$
\left|v_{1}(x)\right| \leq C h^{1-3 N / 2} \exp \left(-\frac{|\Lambda(x)|-\sqrt{h} / 2}{h} \mu^{h}\right) .
$$

Now, we estimate the integral of the square of this expression over the complement of $\mathscr{V}[\sqrt{h}]$. We have to consider two regions: where $d(x, \Gamma) \geq \bar{\lambda}$, there exists a constant $K$ such that

$$
d(x, \Gamma) \geq \max (\bar{\lambda}, K|x|) .
$$

Therefore, the contribution on this region is estimated as

$$
\left(\int_{d(x, \Gamma) \geq \bar{\lambda}}\left|v_{1}(x)\right|^{2} d x\right)^{1 / 2} \leq C h^{1-3 N / 2} \exp \left(-\frac{\bar{\lambda}}{K h} \mu^{h}\right) .
$$

On the remaining region,

$$
\begin{aligned}
& \int_{\sqrt{h} \leq d(x, \Gamma) \leq \lambda} \exp \left(-2 \frac{d(x, \Gamma)-\sqrt{h} / 2}{h} \mu^{h}\right) d x \\
& \leq C \int_{\sqrt{h}}^{\bar{\lambda}} \exp \left(-\frac{\lambda-\sqrt{h} / 2}{h} \mu^{h}\right) d \lambda \\
& \leq C h \exp \left(-\mu^{h} / \sqrt{h}\right) .
\end{aligned}
$$

The gradient verifies an estimate of the same nature up to a power of $h$; by adjusting the constant in the exponentially small term, we can conclude the proof.

5.3. Reduction to one dimension. We have the relations

$$
\begin{aligned}
\chi^{h}= & R^{h}\left[\zeta^{h}\right]=\frac{\int\left(h^{2}\left|\nabla \zeta^{h}\right|^{2}+V^{h}\left|\zeta^{h}\right|^{2}\right) d x}{\int\left|\zeta^{h}\right|^{2} d x} \\
& \geq \frac{\int_{\mathscr{V}[\sqrt{h}]}\left(h^{2}\left|\nabla \zeta^{h}\right|^{2}+V^{h}\left|\zeta^{h}\right|^{2}\right) d x}{\int\left|\zeta^{h}\right|^{2} d x} \\
& =\frac{\int_{\mathscr{V}[\sqrt{h}]}\left(h^{2}\left|\nabla \zeta^{h}\right|^{2}+V^{h}\left|\zeta^{h}\right|^{2}\right) d x}{\int_{\mathscr{V}[\sqrt{h}]}\left|\zeta^{h}\right|^{2} d x} \frac{\int_{\mathscr{V}[\sqrt{h}]}\left|\zeta^{h}\right|^{2} d x}{\int\left|\zeta^{h}\right|^{2} d x}
\end{aligned}
$$


Let $|b|$ be smaller than or equal to $\bar{\lambda}$, and let $y$ belong to $H^{1}(\mathscr{V}[b])$. A restriction of the Rayleigh quotient is defined by

$$
R^{h}[y, b]=\frac{\int_{\mathscr{V}[b]}\left(h^{2}|\nabla y|^{2}+V^{h}|y|^{2}\right) d x}{\int_{\mathscr{V}[b]}|y|^{2} d x} .
$$

This Rayleigh quotient corresponds to a Neumann problem for $-h^{2} \Delta+V^{h}$ over $\mathscr{V}[b]$.

As $\zeta^{h}$ is normalized, relations (5.19) and Proposition 5.4 imply that

$$
R^{h}\left[\zeta^{h}\right] \geq\left(1-k^{\prime} e^{-k / \sqrt{h}}\right) R^{h}\left[\zeta^{h}, \sqrt{h}\right] .
$$

Here, and by an abuse of notation, we denote $\zeta^{h}$ and its restriction to $\mathscr{V}[\sqrt{h}]$ by the same symbols. In particular,

$$
R^{h}\left[\zeta^{h}\right] \geq\left(1-k^{\prime} e^{-k / \sqrt{h}}\right) \inf _{y \neq 0} R^{h}[y, \sqrt{h}] .
$$

Apply the change of variables (5.10), and let $z(\Lambda(x) / h, S(x))=y(x)$. Then $R^{h}[y, \sqrt{h}]$ can be rewritten as

$$
\begin{aligned}
R^{h}[y, \sqrt{h}]= & {\left[\int _ { \Gamma } \int _ { - 1 / \sqrt { h } } ^ { 1 / \sqrt { h } } \left(|(\partial z / \partial \mu)(\sigma, \mu)|^{2}+h^{2}|\nabla S \cdot z(\sigma, \mu)|^{2}\right.\right.} \\
\left.\left.\left.+\phi^{\prime}\left(\Theta^{h}(\mu)\right)|z|^{2}\right)\right) J(h \mu, \sigma) d \mu d \sigma\right] & \\
& \cdot\left[\int_{\Gamma} \int_{-1 / \sqrt{h}}^{1 / \sqrt{h}} z(\mu, \sigma)^{2} d \mu d \sigma\right]^{-1} .
\end{aligned}
$$

We now define one-dimensional Rayleigh quotients depending over $\sigma$ : For a function $y \in H^{1}(-b, b)$, let

$$
\hat{R}^{h}[y, b, \sigma]=\frac{\int_{-b}^{b}\left(|(\partial y / \partial \mu)|^{2}+\phi^{\prime}(\Theta(\mu))|y|^{2}\right) J(h \mu, \sigma) d \mu}{\int_{-b}^{b}|y|^{2} J(h \mu, \sigma) d \mu} .
$$

We have replaced $\boldsymbol{\Theta}^{h}$ by $\boldsymbol{\theta}$ because we are interested in intervals $[-b, b]$ such that $b \leq \bar{\lambda} / 2 h$.

This Rayleigh quotient corresponds to a Neumann problem on $[-b, b]$, with slowly varying coefficients. More precisely, the operator is

$$
y \mapsto-\frac{1}{J(h \mu, \sigma)} \frac{d}{d \mu}\left(J(h \mu, \sigma) \frac{d y}{d \mu}\right)+\phi^{\prime}(\boldsymbol{\theta}) y,
$$

with Neumann conditions at $\pm b$. As is well known, all the eigenvalues of such a problem are isolated and of finite multiplicity. The smallest eigenvalue of (5.24) with $b=1 / \sqrt{h}$ will be denoted $\eta_{1}^{h}(\sigma)$. It is known to be simple, and the corresponding eigenvector can be taken to be strictly positive over $[-1 / \sqrt{h}, 1 / \sqrt{h}]$. The second eigenvalue will be denoted $\eta_{2}^{h}(\sigma)$. In particular,

$$
\eta_{1}^{h}(\sigma)=\inf _{y \neq 0} \hat{R}^{h}[y, 1 / \sqrt{h}, \sigma] .
$$


When $h=0$, the Rayleigh quotient $\hat{R}^{0}[., b, \sigma]$ does not depend over $\sigma$ and corresponds exactly to the Neumann problem on $[-b, b]$ for

$$
y \mapsto-\frac{d^{2} y}{d \mu^{2}}+\phi^{\prime}(\boldsymbol{\Theta}) y .
$$

From now on, we shall write $\bar{R}^{h}[y]$ instead of $\hat{R}^{0}[y, 1 / \sqrt{h}, \sigma]$.

The proof of the following assertion is clear:

Lemma 5.4. The following estimate holds:

$$
R^{h}[y, \sqrt{h}] \geq \min _{\sigma} \eta_{1}^{h}(\sigma), \forall y \in H^{1}(\mathscr{V}[\sqrt{h}]) .
$$

To prove Theorem 5.1, it remains to prove estimates over $\eta_{1}^{h}(\sigma)$.

5.4. Spectral estimates independent of $\sigma$. Let $W(x)=\phi^{\prime}(\boldsymbol{\theta}(x))$. Define an operator $A^{L}$ by

$$
D\left(A^{L}\right)=\left\{u \in H^{2}(-L, L) \mid u^{\prime}( \pm L)=0\right\} ; \quad A^{L} u=-u^{\prime \prime}+W u .
$$

In this subsection we show that for all $\alpha>0$ the intersection of the spectrum of $A^{L}$ with $\left(-\infty, \phi^{\prime}(1)-\alpha\right)$ converges to the intersection of the spectrum of $A$ with $\left(-\infty, \phi^{\prime}(1)-\alpha\right)$, as $L$ goes to infinity.

Lemma 5.4. Let $\left(\eta^{L}\right)$ be a sequence of eigenvalues of $A^{L}$, with corresponding normalized eigenvectors $v^{L}$; assume that there exists an $\alpha \in(0,1)$ such that for all $L$,

$$
\eta^{L} \leq \bar{V}-\alpha
$$

Then, we can extract a converging subsequence from the sequence $\eta^{L}$ and its limit is an eigenvalue of $A$.

Proof. The ideas of the proof of Lemma 5.3 will be used again but in a slightly different fashion. Let $a$ be a positive number, and let $\delta$ be the characteristic function of $[-a, a]$. Let

$$
f_{1}=\delta(\bar{V}-W) y^{L} ; \quad f_{2}=(1-\delta)(\bar{V}-W) y^{L}
$$

and let $v_{j}$ be the solution of

$$
-v_{j}^{\prime \prime}+\left(\bar{V}-\eta^{L}\right) v_{j}=f_{j}
$$

Then,

$$
v^{L}=v_{1}+v_{2}
$$

In this proof, the letter $C$ will denote different constants which do not depend on $L$.

As in Lemma 5.3,

$$
\left|f_{2}\right|_{2} \leq|(1-\delta)(\bar{V}-W)|_{\infty}=\left|\phi^{\prime}(1)-\phi^{\prime}(\Theta(a))\right| \leq C e^{-\gamma a} .
$$

Therefore,

$$
\int_{-L}^{L}\left(v_{2}^{\prime 2}+v_{2}^{2}\right) \leq C e^{-2 \gamma a}
$$


Let us now estimate $v_{1}$ : The Green function of $-\left(d^{2} / d x^{2}\right)+\mu^{2}$ over $(-L, L)$ with Neumann boundary conditions is given by

$$
G\left(x, x^{\prime}, \mu\right)= \begin{cases}\frac{\cosh \mu\left(x^{\prime}+L\right) \cosh \mu(x-L)}{\mu \sinh (2 \mu L)} & \text { if } x>x^{\prime} \\ \frac{\cosh \mu\left(x^{\prime}-L\right) \cosh \mu(x+L)}{\mu \sinh (2 \mu L)} & \text { if } x<x^{\prime}\end{cases}
$$

We will define

$$
\mu^{L}=\sqrt{\bar{V}-\eta^{L}}
$$

Since $\eta^{L}$ is bounded from below by $\phi^{\prime}(0)$ and from above by $\bar{V}-\alpha, \mu^{h}$ is bounded and bounded away from zero.

The function $v_{1}$ is given by

$$
v_{1}(x)=\int G\left(x, x^{\prime}, \mu^{L}\right) f_{1}\left(x^{\prime}\right) d x^{\prime} .
$$

For $\left|x^{\prime}\right| \leq a$ and $|x| \geq 2 a$, we have

$$
\left|G\left(x, x^{\prime}, \mu^{L}\right)\right| \leq C \exp \left(-\mu^{L}\left|x-x^{\prime}\right|\right) .
$$

Since $v^{L}$ satisfies the equation

$$
-\left(v^{L}\right)^{\prime \prime}=\left(\eta^{L}-W\right) v^{L}
$$

and is normalized, we can see that

$$
\left|v^{L}\right|_{\infty} \leq C L
$$

Therefore, we have the estimate

$$
\left|v_{1}(x)\right| \leq C L^{-1} \int_{-a}^{a} G\left(x, x^{\prime}, \mu^{L}\right) d x^{\prime} \leq C L^{-1} \exp \left(-\mu^{L}|| x|-a|\right) .
$$

A similar estimate holds for the gradient of $v_{1}$, so that

$$
\int_{2 a \leq|x| \leq L}\left(v_{1}^{\prime 2}+v_{1}^{2}\right) d x \leq C L^{-1} \exp \left(-\mu^{L} a\right) .
$$

Therefore, gathering (5.27) and (5.29), we can see that

$$
\int_{2 a \leq|x| \leq L}\left(\left|\left(v^{L}\right)^{\prime}\right|^{2}+\left|v^{L}\right|^{2}\right) d x \leq C L^{-1} \exp \left(-k_{2} a\right) .
$$

Here $k_{2}=\min \left(2 \gamma, \mu^{L}\right)$. In particular, letting $a=L / 4$, we can see that

$$
\max \left\{\left|u^{L}(x)\right|: L / 2 \leq|x| \leq L\right\} \leq C L^{-2} \exp \left(-k_{2} L / 4\right),
$$

so that

$$
\lim _{L \rightarrow \infty} v^{L}( \pm L)=0
$$

Since

we have

$$
v^{L}(L)-v^{L}(x)^{2}=2 \int_{x}^{L} v^{L}\left(x^{\prime}\right)\left(v^{L}\right)^{\prime}\left(x^{\prime}\right) d x^{\prime}
$$

$$
\left|v^{L}\right|_{\infty}^{2} \leq\left|v^{L}(L)\right|^{2}+\left|v^{L}\right|_{2}\left|\left(v^{L}\right)^{\prime}\right|_{2}
$$


It follows from (5.31) that

$\left|v^{L}\right|_{\infty}$ is uniformly bounded.

Thus, we can refine $(5.29)$ as

$$
\int_{2 a \leq|x| \leq L}\left(v_{1}^{\prime 2}+v_{1}^{2}\right) d x \leq C \exp \left(-\mu^{L} a\right) .
$$

from which we deduce

$$
\int_{2 a \leq|x| \leq L}\left(\left|\left(v^{L}\right)^{\prime}\right|^{2}+\left|v^{L}\right|^{2}\right) d x \leq C \exp \left(-k_{2} a\right) .
$$

In particular, we can choose for all $\nu>0$ a number $a_{\nu}$ such that for all large enough $L$,

$$
\int_{-a_{\nu}}^{a_{\nu}}\left|u^{L}(x)\right|^{2} d x \geq 1-\nu .
$$

Since $\eta^{L}$ is bounded, we can extract a converging subsequence, still denoted by $\eta^{L}$; its limit will be denoted $\eta$. From (5.28) and (5.32), we deduce that $\left(u^{L}\right)^{\prime \prime}$ is bounded too; therefore, we can extract a subsequence still denoted by $v^{L}$ such that $v^{L}$ (resp. $\left.\left(v^{L}\right)^{\prime}\right)$ converges uniformly on compact sets to $v$ (resp. $\left.v^{\prime}\right)$; from (5.28), we can see that $\left(v^{L}\right)^{\prime \prime}$ converges to $v^{\prime \prime}$ uniformly on compact sets. Relation (5.35) implies morevoer that

$$
v \text { is normalized in } L^{2} \text {. }
$$

If we take the limit of (5.28), we can see that

$$
-v^{\prime \prime}+W v=\eta v
$$

it follows from (5.36) and (5.37) that $\eta$ is an isolated eigenvalue of $A$.

We can deduce an important corollary from Lemma 5.4:

Corollary 5.5. Let $\eta_{1}^{L}$ be the infimum of the spectrum of $A^{L}$. Then, as $L$ goes to infinity, $\eta_{1}^{L}$ goes to zero, and $\eta_{1}^{L}$ is uniformly isolated in the spectrum of $A^{L}$. Proof. We can see by substituting $v=\theta(x)$ in the corresponding Rayleigh quotient that

$$
\limsup _{L \rightarrow \infty} \eta_{1}^{L} \leq 0
$$

But 0 is the infimum of the spectrum of $A$; from Lemma 5.4, we can deduce that

$$
\lim _{L \rightarrow \infty} \eta_{1}^{L}=0 \text {. }
$$

Moreover, $\eta_{1}^{L}$ is isolated in the spectrum of $A^{L}$ : if there were a sequence $\eta_{2}^{l}$ such that $\eta_{2}^{L}$ were an eigenvalue of $A^{L}$ and $\eta_{2}^{L}-\eta_{1}^{L}$ would go to zero, we could consider the normalized eigenvectors $v_{j}^{L}$ corresponding respectively to $\eta_{j}^{L}$ for $j=1$ and $j=2$; then, after possibly extracting a subsequence, $v_{j}^{L}$ would converge to an eigenvector $v^{j}$ of $A$; since $v_{2}^{L}$ would be orthogonal to $v_{1}^{L}, v^{2}$ would be orthogonal to $v^{1}$, which is a contradiction.

5.5. The $\sigma$-dependent spectral problem and conclusion of the proof. The result of Corollary 5.5 still holds in the $\sigma$-dependent case: 
Lemma 5.6. The eigenvalue $\eta_{1}^{h}(\sigma)$ is isolated in the spectrum of the operator (5.24) uniformly with respect to $h, \sigma$.

Proof. Consider the Rayleigh quotient $\hat{R}^{h}[y, 1 / \sqrt{h}, \sigma]$; we can estimate its numerator as follows:

$$
\begin{aligned}
\int_{-1 / \sqrt{h}}^{1 / \sqrt{h}} & {\left[\left|\frac{\partial y}{\partial \mu}\right|^{2}+W(\mu) y^{2}\right] J(h \mu, \sigma) d \mu } \\
\geq & \min _{|\lambda| \leq \sqrt{h}} J(\lambda, \sigma) \int_{-1 / \sqrt{h}}^{1 / \sqrt{h}}\left|\frac{\partial y}{\partial \mu}\right|^{2} d \mu+\min _{|\lambda| \leq \sqrt{h}} J(\lambda, \sigma) \int_{-1 / \sqrt{h}}^{1 / \sqrt{h}} W(\mu) y^{2} d \mu . \\
& +\left(\max _{|\lambda| \leq \sqrt{h}} J(\lambda, \sigma)-\min _{|\lambda| \leq \sqrt{h}} J(\lambda, \sigma)\right) \phi^{\prime}(0) \int_{-1 / \sqrt{h}}^{1 / \sqrt{h}} y^{2}(\mu) d \mu
\end{aligned}
$$

Similarly, the denominator of $\hat{R}^{h}[y, 1 / \sqrt{h}, \sigma]$ can be bounded from above by

$$
\max _{|\lambda| \leq \sqrt{h}} J(\lambda, \sigma) \int_{-1 / \sqrt{h}}^{1 / \sqrt{h}} y^{2}(\mu) d \mu .
$$

Therefore,

$$
\begin{aligned}
\hat{R}^{h}[y, 1 / \sqrt{h}, \sigma] \geq & \frac{\min _{|\lambda| \leq \sqrt{h}} J(\lambda, \sigma)}{\max _{|\lambda| \leq \sqrt{h}} J(\lambda, \sigma)} \bar{R}^{h}[y] \\
& +\phi^{\prime}(0) \frac{\max _{|\lambda| \leq \sqrt{h}} J(\lambda, \sigma)-\min _{|\lambda| \leq \sqrt{h}} J(\lambda, \sigma)}{\max _{|\lambda| \leq \sqrt{h}} J(\lambda, \sigma)}
\end{aligned}
$$

Similarly,

$$
\begin{aligned}
\hat{R}^{h}[y, 1 / \sqrt{h}, \sigma] \leq & \frac{\max _{|\lambda| \leq \sqrt{h}} J(\lambda, \sigma)}{\min _{|\lambda| \leq \sqrt{h}} J(\lambda, \sigma)} \bar{R}^{h}[y] \\
& +\phi^{\prime}(1) \frac{\max _{|\lambda| \leq \sqrt{h}} J(\lambda, \sigma)-\min _{|\lambda| \leq \sqrt{h}} J(\lambda, \sigma)}{\min _{|\lambda| \leq \sqrt{h}} J(\lambda, \sigma)} .
\end{aligned}
$$

Since $\Gamma$ is compact, we obtain the conclusion by virtue of Lemma 5.6.

Observe that this lemma gives us only an estimate of the type

$$
\eta_{1}^{h}(\sigma) \geq C \sqrt{h}
$$

which is not enough to conclude.

We display now an approximate eigenfunction $w^{h}$ of the operator (5.24) corresponding to an approximation of its lowest eigenvalue. This approximation will be an $O\left(h^{2}\right)$ :

Lemma 5.8. Let $z$ be the unique solution of

$$
-z^{\prime \prime}+W z=\theta^{\prime}
$$

which is orthogonal to $\theta$. Let $\omega^{h}$ be a $C^{\infty}$ even function over $\mathbb{R}$, nondecreasing on $\mathbb{R}^{+}$, such that

$$
\omega^{h}= \begin{cases}1, & \text { on }[0,1 / \sqrt{h}-1], \\ 0, & \text { on }[1 / \sqrt{h},+\infty) .\end{cases}
$$


Then

$$
w^{h}(\mu, \sigma)=\left[\theta+h \frac{J_{\lambda}(0, \sigma)}{J(0, \sigma} z\right] \omega^{h}(\mu)
$$

satisfies the Neumann conditions at $\pm 1 / \sqrt{h}$ and

$$
-\frac{1}{J(h \mu, \sigma)} \frac{\partial}{\partial \mu}\left(J(h \mu, \sigma) \frac{\partial w^{h}}{\partial \mu}\right)+W w^{h}=\rho^{h},
$$

where

$$
\left(\int_{-1 / \sqrt{h}}^{1 / \sqrt{h}}\left|\rho^{h}\right|^{2} d \mu\right)^{1 / 2} \leq C h^{2}
$$

Proof. Let us define

$$
\tilde{w}^{h}=\theta+h \frac{J_{\lambda}(0, \sigma)}{J(0, \sigma)} z
$$

we start by computing

$$
\begin{aligned}
\tilde{\rho}^{h}= & \left.\frac{1}{J(h \mu, \sigma)} \frac{\partial}{\partial \mu}(J(h \mu, \sigma)) \frac{\partial \tilde{w}^{h}}{\partial \mu}\right)+W \tilde{w}^{h} \\
= & -\frac{1}{J(h \mu, \sigma)} J_{\lambda}(h \mu, \sigma) \frac{\partial \tilde{w}^{h}}{\partial \mu}-\frac{\partial^{2} \tilde{w}^{h}}{\partial \mu^{2}}+W \tilde{w}^{h} \\
= & -\frac{J_{\lambda}(h \mu, \sigma)}{J(h \mu, \sigma)}\left(\theta^{\prime}+h \frac{J_{\lambda}(0, \sigma)}{J(0, \sigma)} z^{\prime}\right)-\theta^{\prime \prime}--h \frac{J_{\lambda}(0, \sigma)}{J(0, \sigma)} z^{\prime \prime} \\
& +W \theta+h W \frac{J_{\lambda}(0, \sigma)}{J(0, \sigma)} z .
\end{aligned}
$$

According to the definition of $z$ we obtain

$$
\tilde{\rho}^{h}=h \theta^{\prime}\left[\frac{J_{\lambda}(0, \sigma)}{J(0, \sigma)}-\frac{J_{\lambda}(h \mu, \sigma)}{J(h \mu, \sigma)}\right]-h^{2} \frac{J_{\lambda}(h \mu, \sigma)}{J(h \mu, \sigma)} \frac{J_{\lambda}(0, \sigma)}{J(0, \sigma)} z^{\prime} .
$$

The mean value formula implies that for each $\mu$ there is a $\mu^{\prime}$ such that

$$
\frac{J_{\lambda}(0, \sigma)}{J(0, \sigma)}-\frac{J_{\lambda}(h \mu, \sigma)}{J(h \mu, \sigma)}=h \mu \frac{J_{\lambda}\left(h \mu^{\prime}, \sigma\right)^{2}-J\left(h \mu^{\prime}, \sigma\right) J_{\lambda \lambda}\left(h \mu^{\prime}, \sigma\right)}{J\left(h \mu^{\prime}\right)^{2}} .
$$

The expression

$$
\left|\frac{J_{\lambda}(\lambda, \sigma)^{2}-J(\lambda, \sigma) J_{\lambda \lambda}(\lambda, \sigma)}{J(\lambda, \sigma)^{2}}\right|
$$

is bounded over $[-1 / \sqrt{h}, 1 / \sqrt{h}]$ by some constant $C$ independently of $h$. Therefore

$$
\begin{aligned}
& \left(\int_{-1 / \sqrt{h}}^{1 / \sqrt{h}}\left|\tilde{\rho}^{h}\right|^{2} d \mu\right)^{1 / 2} \leq h^{2} C\left(\int \theta^{\prime 2} \mu^{2} d \mu\right)^{1 / 2} \\
& +h^{2}\left(\int z^{\prime 2} d \mu\right)^{1 / 2} \max _{[-\sqrt{h}, \sqrt{h}]}\left|\frac{J_{\lambda}(\lambda, \sigma)}{J(\lambda, \sigma)} \frac{J_{\lambda}(0, \sigma)}{J(0, \sigma)}\right| .
\end{aligned}
$$


We had to truncate $\tilde{w}^{h}$ in order to satisfy the Neumann boundary conditions. Therefore

$$
\begin{aligned}
& \left.-\frac{1}{J(h \mu, \sigma)} \frac{\partial}{\partial \mu}(J(h \mu, \sigma)) \frac{\partial w^{h}}{\partial \mu}\right)+W w^{h} \\
& \quad=\omega^{h} \tilde{\rho}^{h}+\left(\frac{J_{\lambda}(h \mu, \sigma)}{J(h \mu, \sigma)} \frac{\partial \omega}{\partial \mu}-\frac{\partial^{2} \omega}{\partial \mu^{2}}\right) \tilde{w}^{h}-2 \frac{\partial \omega}{\partial \mu} \frac{\partial}{\partial \mu} \tilde{w}^{h} .
\end{aligned}
$$

The extra terms have their support in $[-1 / \sqrt{h},-1 / \sqrt{h}+1] \cup[1 / \sqrt{h}-1,1 / \sqrt{h}]$. Let us show that $z(x)$ and $z^{\prime}(x)$ decrease exponentially with $|x|$. We know that there is a constant $C$ such that $\left|\theta^{\prime}(x)\right| \leq C \exp (-\beta|x|)$ (see (1.6)). By definition, $z$ is bounded in $L^{2}$ and $|z|_{2} \leq C\left|\theta^{\prime}\right|_{2}$; from (5.40), we have $-z^{\prime \prime}=\theta^{\prime}-W z$ and therefore

$$
\left|z^{\prime \prime}\right|_{2} \leq\left|\theta^{\prime}\right|_{2}\left(1+C|W|_{\infty}\right) .
$$

In particular, $z$ is bounded. Let $L$ belong to $\left(0, \phi^{\prime}(1)\right)$; we can rewrite (5.40) as

$$
-z^{\prime \prime}+(W-L)_{+} z+L z=\theta^{\prime}+(W-L)_{-} z .
$$

The right-hand side of this equation is the sum of the exponentially decreasing function $\theta^{\prime}$ and of a bounded function with compact support. Therefore, there exists a (possibly different) $C$ such that

$$
\left|\theta^{\prime}(x)+(W-L)_{-}(x)-z(x)\right| \leq C e^{-\beta|x|} .
$$

Let

$$
\zeta=C^{\prime} e^{-\gamma|x|} \quad \text { with } \gamma \leq \beta
$$

then

$$
\begin{aligned}
-\zeta^{\prime \prime} & +L \zeta+(W-L)_{+} \zeta \\
& =-C^{\prime} \gamma^{2} e^{-\gamma|x|}+L C^{\prime} e^{-\gamma|x|}+2 \gamma C \delta(x)+(W-L)_{+} C^{\prime} e^{-\gamma|x|} \\
& \geq C e^{-\beta|x|}
\end{aligned}
$$

provided that $C^{\prime}\left(L-\gamma^{2}\right) \geq C$. It is clearly possible to find $\gamma$ and $C^{\prime}$ such that these conditions hold. Therefore $\zeta$ is a positive global supersolution and by the maximum principle, $|z(x)| \leq \zeta(x)$, i.e.,

$$
|z(x)| \leq C^{\prime} e^{-\gamma|x|} .
$$

We need an analogous estimate for $\zeta^{\prime}$ : to this end, we differentiate (5.40):

$$
-z^{\prime \prime \prime}+W z^{\prime}=\theta^{\prime \prime}-W^{\prime} z
$$

The same argument applies, by virtue of (1.5) and (5.43). Concluding, in (5.42), the first term is bounded in $L^{2}$ by $(5.41)$, the extra terms can be estimated in $L^{2}$ norm by $C \exp (-\gamma / \sqrt{h})$ (with possibly a different $\gamma$ ). The lemma is proved.

To conclude, we need a perturbation result for isolated eigenvalues of selfadjoint operators: 
Lemma 5.9. For $h^{*}>0$, let $\left\{A^{h}\right\}_{0<h \leq h^{*}}$ be a family of selfadjoint operators in a (complex) Hilbert space $\mathscr{H}$, with norm $|\cdot|$ and scalar product $(.$, .).

We assume that

(i) there exists a complex number $\mu$ and a positive number $\alpha$ such that, for all $h \in\left(0, h^{*}\right), A^{h}-\zeta$ has a bounded inverse if $|\zeta-\mu|=\alpha$;

(ii) there exist families of vectors $\left\{w^{h}\right\},\left\{\rho^{h}\right\}$ and of complex numbers $\left\{\lambda^{h}\right\}$ such that for all $h$

$$
\left\{\begin{array}{l}
A^{h} w^{h}=\lambda^{h} w^{h}+\rho^{h}, \quad\left|\lambda^{h}-\mu\right|<\alpha \\
\left|w^{h}\right|=1 \text { and } \lim _{h \rightarrow 0}\left|\rho^{h}\right|=0
\end{array}\right.
$$

(iii) for all $h, A^{h}$ has a unique eigenvalue $\mu^{h}$ in the disc $B(\mu ; \alpha)$ of center $\mu$ and radius $\alpha$. Then

$$
\lambda^{h}-\mu^{h}=O\left(\left|\rho^{h}\right|\right) .
$$

Proof. Let $P^{h}$ be the projection operator on $\operatorname{ker}\left(A^{h}-\mu^{h}\right)$, given by

$$
P^{h}=\frac{1}{2 \pi i} \int_{\gamma}\left(\zeta-A^{h}\right)^{-1} d \zeta
$$

where $\gamma$ is the circle of center $\mu$ and radius $\alpha$ :

$$
\gamma:[0,2 \pi) \mapsto \mu+\alpha e^{i t}
$$

Since

$$
\left(\lambda^{h}-\zeta\right) w^{h}=A^{h} w^{h}-\zeta w^{h}-\rho^{h}
$$

we can write

$$
w^{h}=\frac{A^{h}-\zeta}{\lambda^{h}-\zeta} w^{h}-\frac{\rho^{h}}{\lambda^{h}-\zeta} .
$$

Therefore

$$
P^{h} w^{h}=-\frac{1}{2 \pi i} \int_{\gamma} \frac{w^{h}}{\lambda^{h}-\zeta} d \zeta+\frac{1}{2 \pi i} \int_{\gamma} \frac{\left(A^{h}-\zeta\right)^{-1} \rho^{h} d \zeta}{\lambda^{h}-\zeta}
$$

Here the first integral equals $w^{h}$ by Cauchy's theorem, the second is $O\left(\left|\rho^{h}\right|\right)$, since $\left|\left(A^{h}-\zeta\right)^{-1}\right|,\left|\lambda^{h}-\zeta\right|^{-1}$ are uniformly bounded by a constant for $\zeta \in \gamma$ and $h \in\left(0, h^{*}\right)$. It follows that $P^{h} w^{h}-w^{h}=\tilde{\rho}^{h}$ where $\left|\tilde{\rho}^{h}\right|=O\left(\left|\rho^{h}\right|\right)$. On the other hand, if $z^{h} \in \operatorname{ker}\left(A^{h}-\mu^{h}\right)$, and $z^{h}$ is normalized:

$$
P^{h} w^{h}=\left(w^{h}, z^{h}\right) z^{h}
$$

hence

$$
\left(w^{h}, z^{h}\right) z^{h}-w^{h}=\tilde{\rho}^{h} .
$$

We multiply this relation scalarly by $w^{h}$ :

$$
\left|\left(w^{h}, z^{h}\right)\right|^{2}-1=\left(\tilde{\rho}^{h}, w^{h}\right),
$$

which proves that

$$
\lim _{h \rightarrow 0}\left|\left(w^{h}, z^{h}\right)\right|=1 .
$$


Multiplying scalarly (5.45) by $z^{h}$ gives

$$
\left(\mu^{h}-\lambda^{h}\right)\left(w^{h}, z^{h}\right)=\left(z^{h}, \rho^{h}\right) .
$$

Taking into account (5.47), we immediately obtain (5.46).

Proof of Theorem 5.1. From Lemmas 5.5, 5.6, 5.8 we see that $\eta_{1}^{h}(\sigma)$ is isolated uniformly in $\sigma, h$. Then we may apply Lemma 5.9 to the following situation: $A^{h}$ is the operator defined in (5.24), $w^{h}, \rho^{h}$ are as specified in Lemma 5.8, $\lambda^{h}=0, \mu=0, \mu^{h}=\eta_{1}^{h}(\sigma)$. We conclude that $\eta_{1}^{h}(\sigma)$ is $O\left(h^{2}\right)$ uniformly in $\sigma$. By the results of subsections 5.1, 5.3, $\eta_{1}^{h}(\sigma)$ is a lower bound for the spectrum of $\mathscr{A}^{h}$. The last statement is proved observing that, since $v^{1}=0$, $\tilde{u}^{q, h}$ differs from $\tilde{u}^{0, h}$ by terms which are $O\left(h^{2}\right)$ (in any norm). The proof is complete.

So far, we have understated the dependence on the (slow) time $s$. As a matter of fact, $\Gamma$ and $V^{h}$ depend smoothly on $s$ when $s$ varies in the compact interval $\left[0, T^{*}\right]$, so that Theorem 5.1 holds uniformly in time.

\section{COMPARISON WITH THE EXACT SOLUTION}

Now that we have the spectral estimates of the previous section, and the precise asymptotic expansions of $\S 4$, we are able to compare the exact solution of (2.2) and its asymptotic expansion, by means of the following result:

Theorem 6.1. Assume that $u$ satisfies

$$
u_{t}-h^{2} \Delta u+\phi(u)=\rho
$$

with initial data

$$
u(x, 0)=u^{0, h}(x, 0) .
$$

Assume that $v$ is the (exact) solution of

$$
v_{t}-h^{2} \Delta v+\phi(v)=0,
$$

with initial data

$$
v(x, 0)=v_{0}(x) \text {. }
$$

If, for $t \leq T^{*} h^{-2}$, the remainder $\rho$ can be estimated by

$$
|\rho(t)|_{2} \leq C_{2} h^{q_{2}}, \quad|\rho(t)|_{\infty} \leq C_{\infty} h^{q_{\infty}}
$$

and the difference between the initial data by

$$
\left|u_{0}-v_{0}\right|_{2} \leq \tilde{C}_{2} h^{q_{2}-2}, \quad\left|u_{0}-v_{0}\right|_{\infty} \leq \tilde{C}_{\infty} h^{q_{\infty}-2},
$$

and if $q_{2}>\max (6,4+N / 2)$ and $q_{\infty} \geq 4$, then, the following estimate hold for $t \leq T^{*} h^{-2}$, and for small enough $h$ :

$$
|u(t)-v(t)|_{2} \leq C_{2}^{\prime} h^{q_{2}-2}, \quad|u(t)-v(t)|_{\infty} \leq C_{\infty}^{\prime} h^{\min \left(q_{\infty}-2, q_{2}-4\right)} .
$$

Proof. Let us denote $w=u-v$, so that

$$
\begin{aligned}
w_{t}-h^{2} \Delta w+\phi^{\prime}(u) w & =v_{t}-h^{2} \Delta v+\phi^{\prime}(u) v-u_{t}+h^{2} \Delta u-\phi^{\prime}(u) u \\
& =-\rho-\phi(v)+\phi(u)+\phi^{\prime}(u)(v-u) .
\end{aligned}
$$


If we set

$$
\psi(u, w)= \begin{cases}\frac{\phi(u+w)-\phi(u)-\phi^{\prime}(u) w}{w^{2}} & \text { if } w \neq 0 \\ 2 \phi^{\prime \prime}(u) & \text { otherwise }\end{cases}
$$

the function $w$ satisfies the partial differential equation

$$
w_{t}-h^{2} \Delta w+\phi^{\prime}(u) w=-\rho-\psi(u, w) w^{2} .
$$

By construction, $\psi$ is continuous in $u$ and $w$; as $u$ and $w$ are bounded, for all time $t \leq T^{*} h^{-2},|\psi(u, w)|_{\infty}$ is bounded for $t \leq T^{*} h^{-2}$ by a constant. We denote by $\alpha(t)$ the $L^{2}$ norm of $u(t)$, and by $\omega(t)$ its $L^{\infty}$ norm.

According to Theorem 5.1, there exists a constant $K$ such that for $t \leq$ $T^{*} h^{-2}$,

$$
\left(-h^{2} \Delta w+\phi^{\prime}(u) w, w\right) \geq-K h^{2} \alpha^{2} .
$$

Therefore, we have the differential inequality

$$
\dot{\alpha}(t) \leq K h^{2} \alpha(t)+|\rho(t)|_{2}+|\psi(u, w)|_{\infty} \alpha(t) \omega(t) .
$$

On the other hand, we may write

$w(., t)=\mathscr{E}\left(., h^{2} t\right) * w_{0}-\int_{0}^{t} \mathscr{E}\left(.,(t-s) h^{2}\right) *\left[\rho+\psi(u, w) w^{2}+\phi^{\prime}(u) w\right](s) d s$, which yields

$\omega(t) \leq\left|w_{0}\right|_{\infty}+\int_{0}^{t}|\rho(s)|_{\infty} d s+\int_{0}^{t}\left|\mathscr{E}\left(.,(t-s) h^{2}\right) *\left[\psi(u, w) w^{2}+\phi^{\prime}(u) w\right](s)\right|_{\infty} d s$.

Let us compute $|\mathscr{E}(., t)|_{2}$ :

$$
\begin{aligned}
|\mathscr{E}(., t)|_{2} & =\left(\int(4 \pi t)^{-N} \exp \left(-x^{2} / 2 t\right) d x\right)^{1 / 2} \\
& =\left(\int(4 \pi t)^{-N} \exp \left(-|y|^{2} / 2\right) t^{N / 2} d y\right)^{1 / 2} \\
& =C(N) t^{-N / 4} .
\end{aligned}
$$

We have to distinguish between three cases: $N<4, N=4$ and $N>4$.

(i) $N<4$. We denote by $C_{\psi}$ and $C_{\phi}$ respectively the upper bounds of $|\psi(u, w)(t)|_{\infty}$ and $\left|\phi^{\prime}(u)(t)\right|_{\infty}$. From (6.3), we deduce that

$$
\begin{aligned}
\omega(t) & \leq \tilde{C}_{\infty} h^{q_{\infty}-2}+t C_{\infty} h^{q_{\infty}}+\max _{s \in[0, t]}\left(\omega(s) \alpha(s) C_{\psi}+\alpha(s) C_{\phi}\right) \int_{0}^{t} \frac{C(N) d s}{\left(h^{2}(t-s)\right)^{N / 4}} \\
& \leq \tilde{C}_{\infty} h^{q_{\infty}-2}+t C_{\infty} h^{q_{\infty}}+\max _{s \in[0, t]}\left(\omega(s) \alpha(s) C_{\psi}+\alpha(s) C_{\phi}\right) h^{-N / 2} \frac{t^{1-(N / 4)}}{1-(N / 4)}
\end{aligned}
$$

Let $\left[0, t(h) h^{-2}\right]$, be the largest time interval included in $\left[0, T^{*} h^{-2}\right]$ over which $\omega(t) \leq C^{\prime \prime} h^{2}$, where $C^{\prime \prime}$ is a positive number which will be determined later. If $C^{\prime \prime}>\tilde{C}_{\infty}$, a continuity argument shows that $t(h)>0$, but $t(h)$ might be small with $h$. On $\left[0, t(h) h^{-2}\right],(6.2)$ becomes

$$
\dot{\alpha}(t) \leq\left(K+C_{\psi} C^{\prime \prime}\right) h^{2} \alpha(t)+C_{2} h^{q_{2}} .
$$


Let $K^{\prime}=K+C_{\psi} C^{\prime \prime}$; then, a Gronwall lemma yields

$$
\alpha(t) \leq \alpha(0) \exp \left(K^{\prime} h^{2} t\right)+C_{2} h^{q_{2}-2} \frac{\exp \left(K^{\prime} h^{2} t\right)-1}{K^{\prime}} \leq\left(\tilde{C}_{2}+\frac{C_{2}}{K^{\prime}}\right) e^{K^{\prime} T^{*}} h^{q_{2}-2} .
$$

We summarize this inequality by

$$
\alpha(t) \leq C_{2}^{\prime} h^{q_{2}-2},
$$

for $t \leq t(h) h^{-2}$. We substitute this estimate of $\alpha$ in (6.4) and obtain

$$
\omega(t) \leq\left(\tilde{C}_{\infty}+T^{*} C_{\infty}\right) h^{q_{\infty}-2}+\left(C^{\prime \prime} C_{\infty} h^{q_{2}}+C_{\phi} h^{q_{2}-2}\right) C_{2}^{\prime} h^{-2} \frac{T^{* 1-(N / 4)}}{1-(N / 4)} C(N),
$$

and the right-hand side of this inequality is less than $C^{\prime \prime} h^{q_{\infty}-2} \leq C^{\prime \prime} h^{2}$, provided that

$$
\begin{aligned}
q_{2} \geq 6, q_{\infty} \geq 4, \quad C^{\prime \prime} & >T^{*} C_{\infty}+\tilde{C}_{\infty}+2 C_{2}^{\prime} C_{\phi} \frac{T^{*} 1-(N / 4) C(N)}{1-(N / 4)}, \\
h & \leq \min \left(1, \frac{C_{\phi}}{C^{\prime \prime} C_{\infty}}\right) .
\end{aligned}
$$

Under these conditions, it is clear that $t(h)=T^{*}$, by a standard connectedness argument.

(ii) $N=4$. We have to adjust the previous proof because $1 / t$ has a nonintegrable singularity at $t=0$. The first step is to observe that if $t=O(1)$, we deduce from (6.3) the crude estimate

$$
\omega(t) \leq \tilde{C}_{\infty} h^{q_{\infty}-2}+C_{\infty} h^{q_{\infty}}+\int_{0}^{t}\left[C_{\psi} \omega(s)^{2}+C_{\phi} \omega(s)\right] d s .
$$

As we know that $w$ is bounded uniformly in time, an application of Gronwall lemma with constants which do not have to be precise gives

$$
\omega(t) \leq\left(\tilde{C}_{\infty} h^{q_{\infty}-2}+C_{\infty} h^{q_{\infty}}\right) O(1) \quad \text { for } t \leq O(1) .
$$

Assume now that $t \geq 1$. We split the second integral which appears in (6.3). Assume $\gamma>0$, and let $t^{\prime}=t-h^{\gamma}$. Then

$$
\begin{aligned}
\int_{0}^{t}\left|\mathscr{E}\left(.,(t-s) h^{2}\right) *\left[\psi(u, w) w^{2}+\phi^{\prime}(u) w\right](s)\right|_{\infty} d s \\
\leq \int_{0}^{t^{\prime}} \frac{C(4) d s}{h^{2}(t-s)} \max _{s \in[0, t]}\left(\omega(s) \alpha(s) C_{\psi}+\alpha(s) C_{\phi}\right) \\
\quad+\left(t-t^{\prime}\right) \max _{s \in[0, t]}\left(\omega(s)^{2} C_{\psi}+\omega(s) C_{\phi}\right) .
\end{aligned}
$$

We use the notations of the case $N<4$; on the interval $\left[0, t(h) h^{-2}\right],(6.5)$, (6.6) hold and by substitution in (6.8)

$$
\begin{aligned}
\omega(t) \leq & \left(\tilde{C}_{\infty}+T^{*} C_{\infty}\right) h^{q_{\infty}-2}+\left(\log \left(T^{*} h^{-2-\gamma}\right) h^{q_{2}-4} C(4) C^{\prime 2}\left(C_{\phi}+C^{\prime \prime} h^{2} C_{\psi}\right)\right) \\
& +h^{2+\gamma} C^{\prime \prime}\left(C_{\phi}+C_{\psi} C^{\prime \prime} h^{2}\right) .
\end{aligned}
$$

The argument of the previous case shows that if $q_{2}>6, q_{\infty} \geq 4, C^{\prime \prime}$ large enough and $h$ small enough, $t(h)=T^{*}$, and the error estimate

$$
|\omega(t)| \leq C h^{\min \left(q_{\infty}-2, q_{2}-4\right)}
$$

holds true. 
(iii) $N>4$. With the same splitting as in the previous case, we have

$$
\begin{aligned}
\omega(t) \leq & \left.\left(\tilde{C}_{\infty}+T^{*} C_{\infty}\right)\right) h^{q_{\infty}-2} \\
& +h^{q_{2}-2} C(N) C_{2}^{\prime}\left[C_{\phi}+C^{\prime \prime} h^{2} C_{\psi}\right] \frac{h^{\gamma(1-(N / 4))-(N / 2)}}{(N / 4)-1} \\
& +h^{2+\gamma} C^{\prime \prime}\left(C_{\phi}+C_{\psi} C^{\prime \prime} h^{2}\right) .
\end{aligned}
$$

The argument of the previous two cases shows that if $q_{2}>4+(N / 2), q_{\infty} \geq 4$, $\gamma$ small enough, $C^{\prime \prime}$ large enough and for all small enough $h$, we can take $t(h)=T$.

Theorems 4.6, 5.1 and 6.1 show that with $q=5$ terms in the expansion of $u$ for $N \leq 4$, the error between the exact solution and $u^{q, h}$ is small with $h$. The number of necessary terms in the expansion increases linearly with the space dimension. It is clear from the statement of Theorem 6.1 that an arbitrarily high precision can be achieved by increasing $q$.

\section{REFERENCES}

[AcGl] N. I. Achieser and I. M. Glasman, Theorie der linearen Operatoren im Hilbert Raum, H. Deutsch, Thun-Frankfurt/M., 1981.

[AlBa] N. D. Alikakos and P. W. Bates, On the singular limit in a phase-field model, Ann. Inst. H. Poincaré Anal. Non Linéaire 6 (1988), 141-178.

[AlCa] S. Allen and J. Cahn, A microscopic theory for antiphase motion and its application to antiphase domain coarsening, Acta Metall. 27 (1979), 1084-1095.

[An1] S. Angenent, Parabolic curves on surfaces I. Curves with p-integrable curvature, TSR 89-19, Dept. of Math., Univ. of Wisconsin, Madison, November 1988.

[An2] - Parabolic curves on surfaces II. Intersections, blow up and generalized solutions TSR 89-24 Dept. of Math., Univ. of Wisconsin, Madison, January 1988.

[An3] _ On the formation of singularities in the curve shortening flow, preprint, Univ. of Wisconsin, Madison, March 1989.

[AnGu] S. Angenent and M. E. Gurtin, Multiphase thermomechanics with interfacial structure. 2. Evolution of an isothermal interface, preprint, 1988.

[BeJoSu] R. Benzi, G. Jona-Lasinio, and A. Sutera, Stochastically perturbed Landau-Ginzburg equations, J. Statist. Phys. 55 (1989), 505-522.

[Bra] K. A. Brakke, The motion of a surface by its mean curvature, Princeton Univ. Press, Princeton, N.J., 1978.

[Bro] L. Bronsard, Reaction-diffusion equations and motion by mean curvature, Ph.D. Thesis, New York Univ., October 1988.

[BroKo1] L. Bronsard and R. V. Kohn, On the slowness of the phase boundary motion in one space dimension, Comm. Pure Appl. Math. 43 (1990), 983-997.

[BroKo2] __, Motion by mean curvature as the singular limit of Ginzburg-Landau dynamics, preprint, Lefschetz Center for Dynamical Systems, August 1989.

[CaGuSl] J. Carr, M. E. Gurtin, and M. Slemrod, Structured phase transitions on a finite interval, preprint.

[CaPe] J. Carr and R. L. Pego, Metastable patterns in solutions of $u_{t}=\epsilon^{2} u_{x x}-f(u)$, Comm. Pure Appl. Math. (in press).

[Cg] G. Caginalp, Mathematical models of phase boundaries, Material Instabilities in Continuum Mechanics and Related Mathematical Problems, (J. M. Ball, ed.), Clarendon Press, Oxford 1988 , pp. 35-52. 
[CgFi] G. Caginalp and P.C. Fife, Elliptic problems involving phase boundaries satisfying a curvature condition, IMA J. Appl. Math. 38 (1987), 195-217.

[Ch] X. F. Chen, Generation and propagation of the interface for reaction-diffusion equations, IMA preprint 637, Inst. for Math. and its Appl., Univ. of Minnesota, Minneapolis, 1990.

[DaSu] V. G. Danilov and P. Yu. Subochev, Exact one and two phase wave-like solutions of semilinear parabolic equations, preprint, Steklov Institute, Moscow, 1988.

[Dt] D. M. Deturck, Deforming metrics in the direction of their Ricci tensors, J. Differential Geom. 18 (1983) 157-162.

[EvSoSo] L. C. Evans, H. M. Soner, and P. E. Souganidis, The Allen-Cahn equation and generalized motion by mean curvature, preprint, 1990.

[Fi] P. C. Fife, Nonlinear diffusive waves, CBMS Conf. at Little Cottonwood Canyon, Utah, 1987, CBMS Conference Series, 1989.

[FiGi] P. C. Fife and G. S. Gill, The phase-field description of mushy zones, Proc. Conf. on Nonlinear Partial Differential Equations, Provo, Utah, 1987.

[FiHs] P. C. Fife and Ling Hsiao, The generation and propagation of internal layers, Nonlinear Analysis TMA, -

[FiML] P. C. Fife and J. B. McLeod, The approach of solutions of nonlinear diffusion equations to travelling front solutions, Arch. Rational Mech. Anal. 65 (1977), 335-361.

[Fre] M. I. Freidlin, Geometric optics approach for reaction-diffusion equations, SIAM J. Appl. Math. 46 (1986), 222-232.

[Fr] A. Friedman, Partial differential equations of parabolic type, Prentice Hall, Englewood Cliffs, N.J., 1964

[FuHa] G. Fusco and J. K. Hale, Slow-motion manifolds, dormant instability, and singular perturbations, J. Dynamics Differential Equations 1 (1989), 75-94.

[Fu] G. Fusco, A geometric approach to the dynamics of $u_{t}=\varepsilon^{2} u_{x x}+f(u)$ for small $\varepsilon$, preprint, June 1989.

[GaHa] M. Gage and R. S. Hamilton, The heat equation shrinking convex plane curves, J. Differential Geom. 23 (1986), 69-96.

[Gi] P. B. Gilkey, Invariance theory, the heat equation and the Atyiah-Singer index theory, Math. Lecture Series 11, Publish or Perish, Wilmington, Del., 1984.

[Gr] M. A. Grayson, The heat equation shrinks embedded plane curves to round points, J. Differential Geom. 26 (1987) 285-314.

[Gu] M. E. Gurtin, Some results and conjectures in the gradient heory of phase transitions, Inst. for Math. and its Appl., Univ. of Minnesota, Minneapolis, 1988.

[GuMa] M. E. Gurtin and H. Matano, On the structure of the equilibrium phase transitions within the gradient theory of fluids, Quart. Appl. Math. 46 (1988), 301-317.

[GuSMSa] J. D. Gunton, M. San Miguel, and P. S. Sahni, The dynamics of first order phase transitions, Phase Transitions, Vol. 8, Academic Press, London, 1983.

[Ha] R. S. Hamilton, Three manifolds with positive Ricci curvature, J. Differential Geom. 20 (1984), 237-266.

[Hu] G. Huisken, Flow by mean curvature of convex surfaces into spheres, J. Differential Geom. 20 (1984), 237-268.

[Jo] J. Jost, Nonlinear Methods in Riemannian and Kähleriann geometry, DMV Seminar, Bd. 10, Birkhäuser, Basel and Boston, Mass., 1988.

[Ka] Ya. I. Kanel', On the stabilization of solutions of the Cauchy problem for the equations arising in the theory of combustion, Mat. Sb. 59 (1965), 398-413.

[KaTa] T. Kato and H. Tanabe, On the abstract evolution equations, Osaka Math. J. 14 (1962), 107-133.

[KaOh] K. Kawasaki and T. Ohta, Kinetic drumhead model of interface I, Progr. Theor. Phys. 67 (1982), 147-163.

[KoSt] R. V. Kohn and P. Sternberg, Local minimizers and singular perturbations, Proc. Roy. Soc. Edinburgh (in press). 
[Li] J.-L. Lions, Équations différentielles opérationnelles, Springer, Berlin, 1961

[LuMo] S. Luckhaus and L. Modica, The Gibbs-Thompson relation within the gradient theory of phase transitions, preprint, Univ. of Pisa, October 1988.

[Mo] L. Modica, The gradient theory of phase transitions and the minimal interface criterion, Arch. Rational Mech. Anal. 98 (1987), 123-142.

[MoSc1] P. de Mottoni and M. Schatzman, Évolution géometrique d'interfaces, C.R. Acad. Sci. Paris Ser. I 309 (1989), 453-458.

[MoSc2] _ Development of interfaces in N-dimensional space, Proc. Roy. Soc. Edimburgh 116A (1990), 207-220.

[Ne] J. Neu, Private communication.

[No] A. Novick-Cohen Blow up and growth in the directional solidification of dilute binary alloys, preprint, Dept. of Math., Michigan State University, 1987.

[Pr] E. Presutti, Collective behaviour of interacting particle systems, Proc. First World Conf. Bernouilli Society (Tashkent, USSR, 1986), Vol. 1, VNU Scientific Press, Utrecht, 1987, pp. 295-413.

[RuStKe,] J. Rubinstein, P. Sternberg, and J. B. Keller, Fast reaction, slow diffusion and curve shortening, preprint, Stanford Univ., 1987.

[Si] B. Simon, On Positive eigenvalues of one-body Schrödinger operators, Comm. Pure Appl. Math. 22 (1967), 531-538.

[Sp] H. Spohn, private communication.

Mathematiques, Université Lyon 1-Claude-Bernard, 69622 Villeurbanne Cedex, FrANCE

E-mail address: schatz@lan1.univ-lyon1.fr 